\title{
CONTROL TECHNOLOGY AND EXPOSURE ASSESSMENT FOR OCCUPATIONAL EXPOSURE TO BERYLLIUM: BERYLLIUM FACILITY \#1 - COPPER/BERYLLIUM FOUNDRY
}

\author{
PRINCIPAL AUTHORS: \\ Daniel Almaguer, MS \\ Ed Burroughs, Ph.D, CIH, CSP \\ Dave Marlow \\ Li-Ming Lo, Ph.D
}

DATE:

July 2008

FILE NO:

EPHB 326-11a
U.S. DEPARTMENT OF HEALTH AND HUMAN SERVICES
Centers for Disease Control and Prevention
National Institute for Occupational Safety and Health
Division of Applied Research and Technology
4676 Columbia Parkway, R5
Cincinnati, Ohio 45226


SITES SURVEYED:

NAICS:

SURVEY DATE:

SURVEY CONDUCTED BY:
Beryllium Facility \#1

Copper/Beryllium Foundry and Machine Shop

Mid-Western USA

331525

May 14-16, 2007

Dan Almaguer, M.S.

Ed Burroughs, Ph.D, CIH

Dave Marlow

LiMing Lo, Ph.D 


\section{DISCLAIMER}

Mention of company names or products does not constitute endorsement by the Centers for Disease Control and Prevention.

The findings and conclusions in this report are those of the author(s) and do not necessarily reflect the views of the National Institute for Occupational Safety and Health. 


\section{INTRODUCTION}

The National Institute for Occupational Safety and Health (NIOSH), working under an interagency agreement with the Office of Regulatory Analysis of the Occupational Safety and Health Administration (OSHA), conducted a study of occupational exposures in secondary beryllium processing facilities to document engineering controls and work practices affecting those exposures. The performance of a thorough industrial hygiene survey for a variety of individual employers provides valuable and useful information to the public and employers in the industries included in the work. The principal objectives of this study were:

1. To measure full-shift, personal breathing zone exposures to metals including beryllium, cadmium and lead.

2. To evaluate contamination of surfaces in the work areas that could create dermal exposures or allow re-entrainment of metals into the air.

3. To identify and describe the control technology and work practices in use in operations associated with occupational exposures to beryllium, as well as to determine additional controls, work practices, substitute materials, or technology that can further reduce occupational beryllium exposures.

4. To evaluate the use of personal protective equipment in these facilities.

5. To determine the size distribution of airborne particles.

An initial walk-through evaluation was conducted in August 2006, to observe processes and conditions in order to prepare for subsequent testing. An in-depth evaluation was conducted May 14 to 16, 2007, by NIOSH researchers from the Engineering and Physical Hazards Branch, Division of Applied Research and Technology, Cincinnati, Ohio. During this evaluation, two full shifts of environmental monitoring were conducted for the duration of normal plant operations. 


\section{PROCESS DESCRIPTION}

On May $14-16,2007$, NIOSH conducted an in-depth industrial hygiene evaluation and survey at a copper/beryllium foundry that manufactures products for the metal die casting industry. This was the first of three beryllium production facilities selected to investigate worker exposures to beryllium where secondary processing of beryllium products takes place. The purpose of the study was to measure airborne beryllium and heavy metal concentrations in the foundry and machining operations of this facility and to identify and describe the control technology and work practices being used in this facility.

\section{Process Description and Work Practices}

This foundry and machine shop manufactures copper/beryllium products containing from $0.45 \%$ to $2.15 \%$ beryllium, $0.35 \%$ to $0.55 \%$ cobalt, $1.8 \%$ to $2.1 \%$ nickel, with the balance being copper. The facility covers approximately 50,000 square feet and employs approximately 45 workers. The employees operate a sand molding operation, melt shop, shake out, cut off, and grinding areas (see Plant Diagram). Production at this facility is split into 2 areas referred to later in this report as plant 1 and plant 2 . The two foundry areas have 25 employees that work one 10-hour shift, four days per week. The machine shop has 11 employees that work two 8-hour shifts, five days per week. Five employees work in maintenance and the remaining workers are in nonberyllium designated areas of the plant including management and office workers.

\section{Foundry}

Operations at this copper/beryllium foundry involved the production of a mold with associated core(s), the melting and pouring of metal into that mold, and the subsequent shake-out operation where the solid metal casting is released by removing the refractory material of the mold. A number of potential health hazards are associated with each stage of this operation. The focus of our evaluation was on the processes in which workers had potential exposures to beryllium and other metals.

The principle exposures typically associated with mold and core production are silica sand and binders such as isocyanates, urea, phenol and formaldehyde. When sand from the shake-out is re-used in the production of molds, there is the potential for metals from previous castings to be carried into this step of the operation, and for that reason testing for metals was conducted in the mold and core making operations. The second fumace room and pouring operation at this foundry used a permanent mold system which eliminated the sand mold system but was otherwise similar to the first.

The operations believed to pose the greatest potential for exposure to beryllium and other metals in the foundries of facility \#1 (Plants 1 and 2) are the melting and pouring processes. The foundry operations and worker job tasks in Plant \#1 and \#2 are very similar. The furnace operators, pourers, and foundry supervisors are present in the foundry areas of the facility during the entire work shift. Specific tasks involved in melting and pouring, include weigh-out of proper ingredients to produce an alloy containing $1 \%$ or $2 \%$ beryllium, charging the furnace, temperature testing, and the pouring of molten metal into the molds. Each of the tasks has potential for exposure to beryllium and other metals in various forms and particle sizes, as well 
as associated safety hazards. All workers involved in the furnace operations wore half-face air purifying respirators equipped with HEPA filters, fire-proof over coats, safety glasses, and leather gloves.

In the Plant \#2 foundry the top of the furnace was equipped with slotted ventilation which was exhausted through flexible ducting connected at the bottom of the fumace. The furnace was located on a platform capable of being mechanically tilted forward for pouring the molten metal into a pre-heated crucible (see Photo 1) which was attached to an overhead cranc for transport to the mold pouring stations. Using long arm-like poles attached to either side of the crucible transport mechanism, the crucible is tilted forward pouring the molten metal into a trough which leads to the inlet of the molds (see Photo 2). Once the trough is filled, the mold is mechanically tilted upright to allow molten metal to drain into the mold. Above the trough attached to the mold was a slotted hood attached by ducting to a local exhaust ventilation system (also shown in Photo 2).

The fumace operations in foundry of Plant \#1 were very similar to Plant \#2. Main differences included: the furnace could not be mechanically tilted forward for pouring and the crucible transport mechanism was equipped with a LEV system above the crucible.

Measurements of both breathing zone concentrations of metals and determination of area concentrations of metals were conducted in the furnace rooms of both Plants 1 and 2. In addition, particle size distribution was also evaluated in these areas. While there are other potential hazards associated with foundries such as heat stress, infrared radiation, and a variety of safety hazards, this evaluation focused primarily on worker exposures to beryllium and toxic metals.

The shake-out operation has the potential for exposures to beryllium and other metals in the form of small particles when the solidified (but still hot) castings are freed from the molds, as well as potential exposure to the sand, which may contain metals, from contact with the molten alloy. Removal of spurs and similar finishing processes using cut-off wheels or grinders also pose the potential for creating airborne particles of metals. All of these operations were monitored for metals, especially beryllium, in the air and on surfaces where skin contact could occur.

\section{Machine Shop}

After cleaning and de-burring of castings in the shake-out operation, the castings were sent to the machine shop. Processes utilized in the machine shop include: machining, grinding, polishing and buffing, each with the potential to create airborne particles of increasingly small size.

Cutting tools used in machining generally remove metal in relatively large chips or tumings, and tend to produce little respirable particulate. The use of coolants and enclosure of machining operations further reduces this potential. The potential for dermal exposure, however, is significant in machining with beryllium and the coolant both being of concern. Area and personal samples were collected in the machine shop for airborne metals.

Grinding, polishing and buffing all involve the removal of metals from the surface of a casting, but in increasingly smaller amounts. The decrease in mass, however, may be offset by a 
corresponding decrease in particle size that may carry with it an increase in toxicity. Therefore, particle size information was collected in the machine shop area also.

\section{Control Technology}

Most of the operations described above were equipped with some type of local exhaust ventilation (canopy hoods, side draft, slot, etc.) system with fixed or flexible ducting to reduce emissions. Some of the process operations (e.g. Plant 2 furnace and pouring stations) were equipped with a hydraulic system which enabled the process to be lifted and reoriented to allow for pouring of the molten metal (see Photo 1). Workers are present in the area of all the operations described above, and interact with the processes. Visual observations indicated in many cases smoke and dust from these operations moved toward the local exhaust ventilation openings, although there were some operations where this was not the case. Air velocity measurements were made to document the magnitude and direction of air movement at selected processes.

Workers in the machine shop place parts into automated lathes which are enclosed and utilize cutting fluids to contain and control the release of metal particles containing beryllium.

\section{Personal Protective Equipment}

Personal protective equipment utilized throughout this facility included safety glasses, safety shoes and earplugs for hearing protection. Half-mask air-purifying respirators equipped with combination mutli-gases/vapors/P100 cartridges were also used in beryllium designated locations including the two foundry areas, sand molding operation, melt shop, shake out, cut off, and grinding areas. Additionally, when pouring molten metal and certain other operations, workers wore protective jackets, gloves, leg protection and face shields.

\section{SAMPLING AND ANALYTICAL METHODS}

This field study was conducted in accordance with regulations governing NIOSH investigations of places of employment. ${ }^{1}$ Methods used to assess worker exposures in this workplace evaluation included: personal breathing zone and area sampling for metals; particle size sampling; surface wipe sampling to assess surface contamination; and bulk material samples to determine the composition of settled dust. The methods used in this evaluation are described in more detail in the following sections.

\section{A. Workplace Observations}

Information pertinent to process operation and control effectiveness (e.g. control methods, ventilation rates, work practices, use of personal protective equipment, etc.) was collected. Observations regarding work practices and use of personal protective equipment were recorded. Information was obtained from conversations with the workers and management to determine if the sampling day was a typical workday to help place the sampling results in proper perspective. In addition, engineering control information including ventilation flow rates and distance measurements were collected. 


\section{B. Particulate Sampling and Analysis}

Personal breathing zone and general area airborne particulate samples were collected and analyzed using inductively coupled plasrna spectroscopy (ICP) according to NIOSH Method $7300^{2}$ (with modifications) for 31 metals/elements. Samples were collected for as much of the work shift as possible, at a flow rate of 3 liters/minute using a calibrated battery-powered sampling pump (model 224, SKC Inc., Eighty Four, PA) connected via flexible tubing to a 37 $\mathrm{mm}$ diameter filter $(0.8 \mu \mathrm{m}$ pore-size mixed cellulose ester filter) in a 3-piece, clear plastic cassette sealed with a cellulose shrink band.

\section{Particulate Size Sampling - Measurement of Size/Mass Distribution of Airborne Particles}

One of the objectives of this study was to determine the particle size and mass concentration of airborne beryllium particles generated during the manufacturing process. There is substantial evidence that the presence of an ultrafine component increases the toxicity for chronic beryllium disease and possibly other toxic effects. $3,4,5$ The potential hazard for chemical substances present in inhaled air, as suspensions of solid particles or droplets, depends on particle size and the mass concentration because of 1 ) the effects of particle size on the deposition site within the respiratory tract, and 2) the tendency for many occupational diseases to be associated with material deposited in particular regions of the respiratory tract. ${ }^{6}$ For example, the ACGIH recommends particle size-selective TLVs for crystalline silica because of the well established association between silica and respirable mass concentrations. ${ }^{6}$ Because of this association, sizeselective sampling was conducted to collect information on the aerosol size distribution to assist in evaluation of the health hazard. Additionally, the measurement and characterization of airborne particle size and mass distribution in workplace environments can provide useful information about the emission and exposure routes of air contaminants generated; and the data collected can be used to identify appropriate control methods to reduce or eliminate contaminate sources to protect workers.

The measurement of particle size and distribution was accomplished using tbree different instruments and methods. Personal breathing zone and general area air samples were collected using Sioutas cascade impactors to determine particle size distribution. Additionally, a MicroOrifice Unifom Deposit Impactor (MOUDI) and an Aerodynamic Particle Sizer (APS) spectrometer were used to measure the particle size and respirable mass concentrations in the general workplace air.

\section{Sioutas Cascade Impactor Samples}

Personal breathing zone and general area aerosol size distributions were determined using fourstage Sioutas Cascade Impactors (SKC, Inc., Eighty Four, PA), having nominal $50 \%$ cut points of $0.25 \mu \mathrm{m}, 0.5 \mu \mathrm{m}, 1 \mu \mathrm{m}$, and $2.5 \mu \mathrm{m}$ aerodynamic diarneter. The sampling flow rate for these impactors was 9 liters/minute, provided by a calibrated Leland Legacy ${ }^{\mathrm{TM}}$ sampling pump (SKC, Inc., Eighty Four, PA). A $25-\mathrm{mm}$ diameter, $0.8 \mu \mathrm{m}$ pore size PVC filter was used on each stage of the impactor to collect particles. A 37-mm diameter, $5 \mu \mathrm{m}$ pore size PVC filter was used as a backup to collect all particles that were not impacted on the previous four stages. The impactor 
filters were analyzed for 31 metals/elements by ICP in accordance with NIOSH Method 7300 modified for mictowave digestion. ${ }^{2}$

\section{Micro-Orifice Uniform Deposit Impactor (MOUDI) Samples}

The MOUDIs (Model 110, MSP Corp., Minneapolis, MN) were used to determine aerosol size distributions in the general area of several production processes at this facility. The MOUDIs were connected via tubing to a high volume pump operating at a flow rate of 30 liters per minute. The MOUDI consists of a pre-filter to collect particles larger than $18 \mu \mathrm{m}$, ten filter stages in series with nominal cut points of $10 \mu \mathrm{m}, 5.6 \mu \mathrm{m}, 3.2 \mu \mathrm{m}, 1.8 \mu \mathrm{m}, 1.0 \mu \mathrm{m}, 0.56 \mu \mathrm{m}, 0.32 \mu \mathrm{m}$, $0.18 \mu \mathrm{m}, 0.10 \mu \mathrm{m}$, and $0.056 \mu \mathrm{m}$ and a post-filter to collect all remaining particles smaller than $0.056 \mu \mathrm{m}$. At each filter stage particles larger than the cut size are collected by a $47-\mathrm{mm}$ diameter substrate on the impaction plate due to inertial impaction while particles smaller than the cut size follow the airflow streamlines and proceed to the next stage until the final stage filter (37-mm diameter, PTFE, SKC Inc.).

Three different substrates were used in the MOUDIs to collect airborne particulate: Aluminum foil filters, PTFE membrane filters with a 0.5 - $\mu$ m-pore-size manufactured by SKC Inc., and PTFE membrane filters with a 2.0- $\mu \mathrm{m}$-pore-size manufactured by Pall Corp. The two different PTFE membrane filters with different pore sizes and manufactures were used to eliminate sampling bias from collecting materials; and the Aluminum foil filters were used because the accuracy of gravimetric analysis of membrane filters can be affected by environmental humidity and sample transit. ${ }^{7}$ To prevent particle bounce during sampling, a thin layer of silicon spray was applied to the Aluminum foil filters and the filters were baked for a minimum of 2 hours at $100^{\circ} \mathrm{C}$. All the sample filters remained in the balance room for 24 hours before pre-weighing on an electric balance (Model AT20, Mettler-Toledo, Switzerland) to $2 \mu \mathrm{g}$ resolution, stored and transported in Petri dishes before and after sampling.

Three MOUDIs were used in this study to measure the mass distribution of airborne particles at the locations near furnaces and cutting equipment where high particle concentrations were expected. Usually 8-hour sampling is necessary to obtain adequate mass for the following gravimetric analysis. Similar to the preparation steps mentioned above, the filter samples were kept in the Petri dishes after MOUDI sampling, and the post-weighing was conducted in our laboratory after 24-hour conditioning in the balance room. After post-weighing, the PTFE filters were sent to a contract laboratory for the metal analysis.

\section{Aerodynamic Particle Sizer (APS) Samples}

An APS spectrometer (Model 3321, TSI, Shoreview, MN) was used to collect real time particle number measurements at various locations throughout this foundry including the locations where the MOUDI samples were collected. All the APS sampling data were collected by Aerosol Instrument Manager Software for APS Sensors. This instrument is capable of measuring particles ranging from $0.5 \mu \mathrm{m}$ to $20 \mu \mathrm{m}$ at 5.0 liters per minute (lpm) total sampling flow rate including $1.0 \mathrm{lpm}$ aerosol flow and $4.0 \mathrm{lpm}$ sheath flow. A minimum of 10 samples were collected at each sample location with the APS set to run in a one-minute sampling mode. 


\section{Surface Sampling Procedures and Analysis}

Surface sampling is not as useful as airbome contaminant measurements for evaluating exposed dose since there are few criteria for reference, but some comparisons and professional judgments can be made based on the data collected, as discussed below. Surface sampling is useful for evaluating process control and cleanliness and for determining suitability for release of equipment.

Surface wipe samples were collected using Ghost ${ }^{\mathrm{TM}}$ Wipes (Environmental Express, $\mathrm{Mt}$. Pleasant, SC) and Palintest ${ }^{(1)}$ Dust Wipes (Gateshead, United Kingdom) to evaluate surface contamination. These wipe samples were collected in accordance with ASTM Method D 6966$03,{ }^{8}$ except the cardboard template, with a $10-\mathrm{cm}$ by $10-\mathrm{cm}$ square hole was held in place by hand rather than taped in place, to prevent movement during sampling. Wipes were placed in sealable test tube containers for storage until analysis.

Ghost Wipes ${ }^{\text {TM }}$ were sent to the laboratory to be analyzed for metals according to NIOSH Method 7303. ${ }^{9}$ Palintest wipes were analyzed for beryllium using the Quantech Fluorometer (Model FM109515, Bamstead International, Dubuque, Iowa) for spectrofluorometric analysis. ${ }^{10}$

\section{E. Other Measurements}

Bulk material samples were collected and analyzed for 31 metals/elements using NIOSH Method $7300^{2}$ modified for bulk digestion. Ventilation aifflow measurements were collected using a TSI VelociCalc Plus Air Velocity Meter Model 8360. A comprehensive ventilation evaluation of the ventilation systems in this facility is beyond the scope of this study and was not conducted due to the size of the facility, the large number of processes equipped with LEV, and the amount of time that would be required to conduct such an evaluation. 


\section{OCCUPATIONAL EXPOSURE LIMITS AND HEALTH EFFECTS}

In evaluating the hazards posed by workplace exposures, NIOSH investigators use mandatory and recommended occupational exposure limits (OELs) for specific chemical, physical, and biological agents. Generally, OELs suggest levels of exposure to which most workers may be exposed up to 10 hours per day, 40 hours per week for a working lifetime without experiencing adverse health effects. ${ }^{\dagger}$ It is, however, important to note that not all workers will be protected from adverse health effects even though their exposures are maintained below these levels. A small percentage may experience adverse health effects because of individual susceptibility, a pre-existing medical condition, and/or hypersensitivity (allergy). In addition, some hazardous substances may act in combination with other workplace exposures, the general environment, or with medications or personal habits of the worker to produce health effects even if the occupational exposures are controlled at the level set by the exposure limit. Combined effects are often not considered in the OEL. Also, some substances can be absorbed by direct contact with the skin and mucous membranes in addition to being inhaled, thus contributing to the overall exposure. Finally, OELs may change over the years as new information on the toxic effects of an agent become available.

Most OELs are expressed as a time-weighted average (TWA) exposure. A TWA refers to the average exposure during a normal 8- to 10 -hour workday. ${ }^{*}$ Some chemical substances and physical agents have recommended short-term exposure limits (STEL) or ceiling values where there are health effects from higher exposures over the short-term. Unless otherwise noted, the STEL is a 15-minute TWA exposure that should not be exceeded at any time during a workday, and the ceiling limit is an exposure that should not be exceeded at any time, even instantaneously.

In the U.S., OELs have been established by Federal agencies, professional organizations, state and local governments, and other entities. Some OELs are mandatory, legal limits; others are recommendations. The U.S. Department of Labor Occupational Safety and Health Administration (OHSA) Permissible Exposure Limits (PELs) [29 CFR 1910 (general industry); 29 CFR 1926 (construction industry); and 29 CFR 1915, 1917 and 1918 (maritime industry)] are legal limits that are enforceable in workplaces covered under the Occupational Safety and Health $\mathrm{Act}^{11}$ and in Federal workplaces under Executive Order 12196. ${ }^{12}$ NIOSH recommended exposure limits (RELs) are recommendations that are made based on a critical review of the scientific and technical information available on the prevalence of hazards, health effects data, and the adequacy of methods to identify and control the hazards. Recommendations made through 1992 are available in a single compendium; ${ }^{13}$ more recent recommendations are

\footnotetext{
${ }^{\dagger}$ On March 20, 1991, the Supreme Court decided the case of International Union, United Automobile, Aerospace \& Agricultural Implement Workers of America, UAW v. Johnson Controls, Inc., 111 S. Ct. 1196, 55 EPD 40,605. It held that Title VII forbids sex-specific fetal protection policies. Both men and women must be protected equally by the employer.

‡ OSHA PELs, unless otherwise noted, are TWA concentrations that must not be exceeded during any 8 -hour workshift of a 40-hour work-week [NIOSH 1997]. NIOSH RELS, unless otherwise noted, are TWA concentrations for up to a 10-hour workday during a 40-hour workweek [NIOSH 1997]. ACGIH ${ }^{(1)}$ TLVs $^{\text {(B) }}$, unless otherwise noted, are TWA concentrations for a conventional 8-hour workday and 40-hour workweck [ACGIH 2008]
} 
available on the NIOSH Web site (http://www.cdc.gov/niosh). NIOSH also recommends preventive measures (e.g., engineering controls, safe work practices, personal protective equipment, and environmental and medical monitoring) for reducing or eliminating the adverse health effects of these hazards. The NIOSH Recommendations have been developed using a weight of evidence approach and formal peer review process. Other OELs that are commonly used and cited in the U.S. include the threshold limit values (TLVs) ${ }^{\circ}$ recommended by the American Conference of Governmental Industrial Hygienists (ACGIH) ${ }^{\circledR}$, a professional organization. ${ }^{6}$ ACGIH-TLVs are considered voluntary guidelines for use by industrial hygienists and others trained in this discipline "to assist in the control of health hazards." Workplace environmental exposure levels (WEELs) are recommended OELs developed by AIHA, another professional organization. WEELs have been established for some chemicals "when no other legal or authoritative limits exist.",14

Employers should understand that not all hazardous chemicals have specific OSHA-PELs and for many agents, the legal and recommended limits mentioned above may not reflect the most current health-based information. However, an employer is still required by OSHA to protect their employees from hazards even in the absence of a specific OSHA-PEL. In particular, OSHA requires an employer to furnish employees a place of employment that is free from recognized hazards that are causing or are likely to cause death or serious physical harm [Occupational Safety and Health Act of 1970, Public Law 91-596, sec. 5(a)(1)]. Thus, NIOSH investigators encourage employers to make use of other OELs when making risk assessment and risk management decisions to best protect the health of their employees. NIOSH investigators also encourage the use of the traditional hierarchy of controls approach to eliminating or minimizing identified workplace hazards. This includes, in preferential order, the use of: (1) substitution or elimination of the hazardous agent, (2) engineering controls (e.g., local exhaust ventilation, process enclosure, dilution ventilation) (3) administrative controls (e.g., limiting time of exposure, employee training, work practice changes, medical surveillance), and (4) personal protective equipment (e.g., respiratory protection, gloves, eye protection, hearing protection).

Both the OSHA PELs and ACGIH ${ }^{\circledR} \mathrm{TLV}^{\circledR}$ address the issue of combined effects of airborne exposures to multiple substances. ${ }^{6,11} \mathrm{ACGH}^{\otimes}$ states:

When two or more hazardous substances have a similar toxicological effect on the same target organ or system, their combined effect, rather than that of either individually, should be given primary consideration. In the absence of information to the contrary, different substances should be considered as additive where the health effect and target organ or system is the same. That is, if the sum of

$$
\frac{C_{1}}{T_{1}}+\frac{C_{2}}{T_{2}}+\ldots \frac{C_{n}}{T_{n}} \quad \text { Eqn. } 1
$$

exceeds unity, the threshold limit of the mixture should be considered as being exceeded (where $\mathrm{C}_{1}$ indicates the observed atmospheric concentration and $\mathrm{T}_{1}$ is the corresponding threshold limit...). 


\section{A. Inhalation Exposures}

Metals found in the workplace under investigation range from slightly toxic to extremely toxic by inhalation. While a subset of five primary contaminants have been selected for consideration through the body of this report because of their high toxicity or otber special interest, the occupational exposure limits of all 31 metals/elements quantified in this work are listed in Table 1.

\section{Occupational Exposure Criteria for Beryllium}

The current OSHA PELs for beryllium are 2 micrograms per cubic meter $\left(\mu \mathrm{g} / \mathrm{m}^{3}\right)$ as an 8-hour TWA, $5 \mu \mathrm{g} / \mathrm{m}^{3}$ as a ceiling not to be exceeded for more than 30 minutes at a time, and $25 \mu \mathrm{g} / \mathrm{m}^{3}$ as a peak exposure never to be exceeded. ${ }^{11}$ The current NIOSH Recommended Exposure Limit (REL) for beryllium is $0.5 \mu \mathrm{g} / \mathrm{m}^{3}$ for up to a 10 -hour work day, during a 40-hour workweek. ${ }^{15}$ The current American Conference of Governmental Industrial Hygienists (ACGWH ${ }^{B}$ ) Threshold Limit Value $\left(\mathrm{TLV}^{\mathrm{Q}}\right)^{6}$ is an 8-hr TWA of $2 \mu \mathrm{g} / \mathrm{m}^{3}$, and a Short Term Exposure Limit (STEL) of $10 \mu \mathrm{g} / \mathrm{m}^{3}$.

Beryllium has been designated a Group1, known human carcinogen, by the Intermational Agency for Rescarch on Cancer (IARC 1993). In 2006 the ACGIH published a Notice of Intended Change (NIC) to reduce the $\mathrm{TLV}^{\circledR}$ for beryllium from 0.002 milligrams per cubic meter $\left(\mathrm{mg} / \mathrm{m}^{3}\right)$ to $0.00005 \mathrm{mg} / \mathrm{m}^{3}$ or 0.05 micrograms per cubic meter $\left(\mu \mathrm{g} / \mathrm{m}^{3}\right)$ and reducing the STEL from 0.01 $\mathrm{mg} / \mathrm{m}^{3}$ of $0.002 \mathrm{mg} / \mathrm{m}^{3}$ based upon studies investigating both chronic beryllium disease (CBD) and beryllium sensitization (BeS). ${ }^{3}$

\section{Occupational Exposure Criteria for Copper}

In this facility copper metal is present in two physical states, copper fume and copper dust, and each has a separate environmental criteria. The NIOSH-REL ${ }^{15}$ and OSHA-PEL ${ }^{11}$ for copper fume are $0.1 \mathrm{mg} / \mathrm{m}^{3}$, while the ACGIH-TLV is $0.2 \mathrm{mg} / \mathrm{m}^{3}$ as an eight-hour TWA. ${ }^{6}$ Inhalation of copper fume has resulted in irritation of the upper respiratory tract, metallic taste in the mouth, and nausea. Exposure has been also associated with the development of metal fume fever. ${ }^{13,16}$

The NIOSH-REL for copper dust is $1 \mathrm{mg} / \mathrm{m}^{3}$ measured as an $8-10$ hour TWA. ${ }^{15}$ The ACGIHTLV and OSHA-PEL are also $1 \mathrm{mg} / \mathrm{m}^{3}$ measured as an 8-hour TWA. ${ }^{6,11}$

\section{B. Surface Contamination Criteria}

Occupational exposure criteria have been discussed above for airborne concentrations of several metals. Surface wipe samples can provide useful information in two circumstances; first, when settled dust on a surface can contaminate the hands and then be ingested when transferred from hand to mouth; and second, if the surface contaminant can be absorbed through the skin and the skin is in frequent contact with the surface. ${ }^{17}$ Although some OSHA standards contain housekeeping provisions which address the issue of surface contamination by mandating that surfaces be maintained as free as practicable of accumulations of the regulated substances, there are currently no surface contamination criteria included in OSHA standards. ${ }^{18}$ The health hazard from these regulated substances results principally from their inhalation and to a smaller extent 
from their ingestion; those substances are by and large "negligibly" absorbed through the skin. ${ }^{17}$ NIOSH RELs do not address surface contamination either, nor do ACGIH TLVs or AIHA WEELs. Caplan stated, "There is no general quantitative relationship between surface contamination and air concentrations..." and that "Wipe samples can serve a purpose in determining if surfaces are as 'clean as practicable'. Ordinary cleanliness would represent totally insignificant inhalation dose; criteria should be based on surface contamination remaining after ordinarily thorough cleaning appropriate for the contaminant and the surface."17 With those caveats in mind, the following paragraphs present guidelines that help to place the results of the surface sampling conducted at this facility in perspective.

\section{Surface Contamination Criteria for Beryllium}

A useful guideline to address the issues of beryllium surface contamination is provided by the U.S. Department of Energy (DOE), where DOE and its contractors are required to conduct routine surface sampling to determine housekeeping conditions wherever beryllium is present in operational areas of DOE/NNSA facilities. ${ }^{3}$ Those facilities must maintain removable surface contamination levels that do not exceed $3 \mu \mathrm{g} / 100 \mathrm{~cm}^{2}$ during non-operational periods. The DOE also has release criteria that must be met before beryllium-contaminated equipment or other items can be released to the general public or released for use in a non-beryllium arca of a DOE facility. These criteria state that the removable contamination level of equipment or item surfaces does not exceed the higher of $0.2 \mu \mathrm{g} / 100 \mathrm{~cm}^{2}$ or the level of beryllium in the soil in the area of release. Removable contamination is defined as "beryllium contamination that can be removed from surfaces by nondestructive means, such as casual contact, wiping, brushing, or washing."

\section{Surface Contamination Criteria for Copper}

NIOSH, OSHA, AIHA and ACGHB have not established occupational exposure limits for Copper on surfaces.

\section{RESULTS AND DISCUSSION}

On May 15 and 16, 2007, air, surface wipe, particle size and bulk material samples were collected in the foundry areas and the machine shop of this copper/beryllium foundry. These samples were analyzed for thirty-one metals/elements (aluminum, antimony, arsenic, barium, beryllium, cadmium, calcium, chromium, cobalt, copper, iron, lanthanum, lead, lithium, magnesium, manganese, molybdenum, nickel, phosphorus, potassium, selenium, silver, strontium, tellurium, thallium, tin, titanium, vanadium, yttrium, zinc, and zirconium) in accordance with NIOSH Method 7303 with modifications. ${ }^{9}$ Because this foundry manufactured copper/beryllium metal products the primary focus of this evaluation was beryllium and copper. Beryllium was the only airborne metal detected that exceeded its' respective occupational exposure criteria. The entire set of sample data for the air, surface wipe, cascade impactor particle size, MOUDI particle size, and bulk material samples for all thirty-one elements are listed in Appendices $A, B, C, D$, and $E$ respectively. 


\section{A. Air Sample Results}

Personal breathing zone and area air sampling results for beryllium and copper are compiled in Table 2 and the entire sample set of 31 elements/metals are presented in Appendix A.

A total of 27 full-shift personal breathing zone and general area air samples for elements/metals were collected on two consecutive days; 24 personal breathing zone samples and three general area air samples. Exposure concentrations were calculated from the analytical results after correcting for the results of field blanks. Because this plant works a ten-hour shift, most air samples were for greater than eight hours duration. The actual sample time (in minutes) is listed along with the airbome beryllium and copper concentrations measured in Table 2.

Beryllium was detected in all samples collected with airborne concentrations ranging from 0.06 $\mu \mathrm{g} / \mathrm{m}^{3}$ to a high of $5.52 \mu \mathrm{g} / \mathrm{m}^{3} ; 16$ of 24 personal samples collected indicated airborne concentrations exceeding the NIOSH REL for beryllium $\left(0.50 \mu \mathrm{g} / \mathrm{m}^{3}\right)$ and seven exceeded the OSHA PEL as described below. Workers in this facility wore half-mask air-purifying respirators equipped with combination mutli-gases/vapors/P100 cartridges which have and assigned protection factor of 10 . However, in one instance the airbome beryllium concentration detected exceeded the maximum use concentration (MUC) of the respirator being used. Specifically, the airbome beryllium concentration measured in the breathing zone of the Plant 1 furnace operator on Day One had a TWA of $5.52 \mu \mathrm{g} / \mathrm{m}^{3}$ (11x the NIOSH-REL and 2.8x the OSHA PEL). Additionally, the beryllium concentration measured in the breathing zone of the Plant 2 furnace operator on Day One had a TWA of $4.72 \mu \mathrm{g} / \mathrm{m}^{3}$ (9.4x above the NIOSH REL and $2.4 \mathrm{x}$ the OSHA PEL). Samples collected the second day indicated that the highest airborne concentration detected in the breathing zone of a furnace operator was $1.16 \mu \mathrm{g} / \mathrm{m}^{3}$ (4x above the NIOSH REL). Two other personal breathing zone samples with airborne beryllium TWA concentrations greater than five times the NIOSH REL were those collected on the saw operator on Day One (2.54 $\mu \mathrm{g} / \mathrm{m}^{3}-5 \mathrm{x}$ the NIOSH REL) and the grinder on Day Two $\left(4.79 \mu \mathrm{g} / \mathrm{m}^{3}-9.6 \mathrm{x}\right.$ the NIOSH REL and $2.4 x$ the OSHA PEL).

Copper was detected in all samples collected; however, because of the different operations evaluated, the copper sampling results have to be compared to two different standards, copper fume and copper dust. Copper fume results ranged from $23.1 \mu \mathrm{g} / \mathrm{m}^{3}$ to a high of $50.4 \mu \mathrm{g} / \mathrm{m}^{3}$ with the highest concentration being 50\% of both the NIOSH REL and the OSHA PEL $\left(100 \mu \mathrm{g} / \mathrm{m}^{3}\right)$. All copper dust concentrations were less than $20 \%$ of both the NIOSH REL and OSHA PEL $\left(1000 \mu \mathrm{g} / \mathrm{m}^{3}\right.$ ) with concentrations ranging from 3.53 to $168 \mu \mathrm{g} / \mathrm{m}^{3}$.

Cobalt was the only other metal which was detected at a significant concentration. The highest airborne cobalt concentration detected was $18.8 \mu \mathrm{g} / \mathrm{m}^{3}$ (38\% of the NIOSH REL of $50 \mu \mathrm{g} / \mathrm{m}^{3}$ ), or $19 \%$ of the less restrictive OSHA PEL of $100 \mu \mathrm{g} / \mathrm{m}^{3}\left(0.1 \mathrm{mg} / \mathrm{m}^{3}\right)$. This concentration was detected on the worker weighing ingredients in Plant 1 ; however, all other cobalt samples were less than $5 \%$ of the NIOSH-REL.

\section{B. Surface Wipe Sample Results}

A total of 29 surface wipe samples were collected on May 14, 15, and 16, 2007. Of the 29 surface wipe samples collected, 21 were analyzed for 31 metals/elements, and the remaining 8 
were analyzed for beryllium only. The analytical results for beryllium (Be), Cadmium (Cd), copper $(\mathrm{Cu})$, lead $(\mathrm{Pb})$ and nickel $(\mathrm{Ni})$ are presented in Table 3; and the entire surface wipe sample data set is presented in Appendix B.

All 29 surface wipe samples had detectable concentrations of beryllium with concentrations ranging from 1.0 to $1200 \mu \mathrm{g} / 100 \mathrm{~cm}^{2}$. Nine of the 21 surface wipe samples indicated detectable concentrations of cadmium ranging from 0.14 to $1.2 \mu \mathrm{g} / 100 \mathrm{~cm}^{2}$. All 21 surface wipe samples indicated detectable concentrations of copper which ranged from 16 to $11,000 \mu \mathrm{g} / 100 \mathrm{~cm}^{2}$. Nineteen of the 21 surface wipe samples had detectable concentrations of lead which ranged from 0.44 to $25 \mu \mathrm{g} / 100 \mathrm{~cm}^{2}$. Sixteen of the 21 surface wipe sample results had detectable concentrations of nickel which ranged from 3.6 to $220 \mu \mathrm{g} / 100 \mathrm{~cm}^{2}$. All 21 surface wipes samples had detectable concentrations of cobalt, with one wipe (on top of small scale in metal weighing area) having a surface concentration of $3500 \mu \mathrm{g} / 100 \mathrm{~cm}^{2}$, however, all other wipe samples ranged from 0.53 to $360 \mu \mathrm{g} / 100 \mathrm{~cm}^{2}$.

\section{Particulate Size/Mass Distribution Results}

One of the objectives of this study was to determine the particle size and mass concentration of airborne beryllium particles generated during the manufacturing process because there is substantial evidence that the presence of an ultrafine component increases the toxicity for chronic beryllium disease and possibly other toxic effects. The results of size-selective sampling indicate that airborne beryllium particles smaller than $2.5 \mu \mathrm{m}$ are present in this work environment and suggest that a portion of the airborne beryllium particles may potentially reach the lower portions of the respiratory tract. The highest total beryllium concentrations were in a sample collected at the cut-off operation on day two of sampling; $33 \%$ of the measured berylliurn was smaller than $2.5 \mu \mathrm{m}$ (impactor stages $B$ to $E$, see Table 4).

The results of particle size measurements collected using the Sioutas cascade impactors, the MOUDI and APS are summarized below and presented in Tables 4 and 5, and Figure 1; and the entire data sets are contained in Appendices C, D and E. The term particle size refers to the aerodynamic size which is defined as the diameter of the spherical particle with a density of $1 \mathrm{~g} / \mathrm{cm}^{3}$ that has the same settling velocity as the particle.

\section{Sioutas Cascade Size-Selective Impactor Results}

The results of size-selective sampling for beryllium $(\mathrm{Be})$ and copper $(\mathrm{Cu})$ using the Sioutas Cascade Impactors are presented in Table 4, while the entire data set for the 31 metals/elements included in the laboratory analyses is presented in Appendix C. A total of 14 size-selective impactor samples were collected during the two days of air sampling; 13 of the 14 samples collected were personal breathing zone air samples and one was an area sample; five of the 13 personal breathing zone samples were collected in hot process areas where one would expect the particulate to be a fume, and 8 of the 13 personal breathing zone samples were collected in areas where the particulate would be a dust. The results presented in Table 4 show the beryllium and copper concentrations measured on each of the five impactor stages and the sum total of all five stages for each sample collected. All samples collected indicated detectable concentrations of both beryllium and copper. These samples indicate measurable quantities of beryllium particles 
smaller than $2.5 \mu \mathrm{m}$ (stages $\mathrm{B}$ to $\mathrm{E}$ ). This tends to suggest that airborne beryllium is present in concentrations that may potentially reach the lower portions of the respiratory tract. The highest total beryllium concentrations detected were in a sample collected at the cut-off operation on day two of sampling and $33 \%$ of the measured beryllium was found in stages $B$ to $E$.

\section{MOUDI Size-Selective Impactor and APS Results}

The MOUDIs size-selective impactor sample results for the 31 metals/elements are presented in Appendix D. Samples collected with the MOUDI size-selective impactor do show measurable concentrations of beryllium in the respirable range, but failed to provide conclusive information about the particle mass distributions, due to either (1) the low airborne particle concentrations at the sample locations selected or (2) the fragile samplers were damaged during unloading at the end of the sample period and/or transit back to the laboratory for the gravimetric analysis.

However, the APS was used to check the number concentrations of airborne particles at the three sampling locations where the MOUDI samples were collected on May 15 and 16, 2007. The sampling locations were designated $\mathrm{A}$ (the furnace room in plant 1 ), $\mathrm{B}$ (the furnace room in plant 2 ), and $C$ (the cutting/grinding area in plant 2). Additionally, the particle concentrations were measured in the office where the NIOSH instrumentation was stored and setup for reference.

The APS data are presented graphically in Figure 1 and are summarized numerically in Table 5. The particle size number concentration curves in Figure 1 show that the particle sizes in this copper/beryllium foundry were concentrated around $0.6 \mu \mathrm{m}$ (mode), except the result for location A on May 16 which showed that larger size particles around $0.8 \mu \mathrm{m}$ were detected. Generally speaking, the same level of airborne particle sizes were found during the regular work shifts throughout the facility.

Summarizing the sampling data shown in Table 5, we found that the particle concentration on May 15 was higher than that on May 16 for the same sampling locations. Because of similar activities conducted at the sampling locations in the two-day survey, the concentration difference could partially result from the other environmental factors such as the ventilation systems and atmospheric conditions. Comparing the particle concentrations found in the different sample locations suggests that working in the fumace rooms is likely to have a higher risk for beryllium exposure than working in the cutting/grinding area.

\section{Bulk Sample Results}

Five bulk dust samples were collected to determine what elements were present in the work place and to what extent. The results for beryllium and copper are listed in Table 6 and the entire data set for the 31 metals/elements are contained in Appendix E. The highest concentrations of beryllium and copper were measured in a dust sample collected behind the furnaces in plant 1 , with concentrations of $130,000(13 \%)$ and $350,000(35 \%) \mathrm{mg} / \mathrm{Kg}$, respectively.

\section{E. Ventilation Measurement Observations/Results}

Considerable air movement was noted throughout both plants, with doors and windows open to promote cross ventilation. Pedestal and wall mounted fans were distributed throughout the 
facility for comfort, although these fans may have increased the amount of metal-containing dust in the air. Some in-wall fans, primarily in the shake-out area, were operating to induce general air flow through the plant, but typical of this type of fan air flow dropped to less than 50 feet per minute (fpm) at distances of 15 to 20 feet from the fan. General exhaust ventilation throughout the facility appeared non-uniform.

Ventilation measurements collected in Plant 1 showed face velocities up to 700 feet per minute (fpm) at the induction furnace hood opening (see Photo 3); the hood opening measured approximately 24 inches by 18 inches. Slot velocities up to $1200 \mathrm{fpm}$ were measured above the pour rack (see Photo 4); slot dimensions were approximately 2 inches by 36 feet. Visual observations confirm that most of the smoke produced during a pour is captured by these slotted exhaust hoods. Ventilation airflow measurements collected in Plant 2 at the mold pouring station showed velocities of 200-300 fpm at the hood duct opening with the molds closed and covers in place (see Photo 2). The canopy measured 2 feet by 4 feet. After a pour air flows less than $100 \mathrm{fpm}$ were measured at the face of the exhaust openings, and observations indicated smoke was not being captured.

Local exhaust ventilation was in place in the furnace areas of both plants and air flow measurements as well as visual observations indicated that much of the emissions from the melting and pouring tasks were captured by that ventilation. There were occasions, however, when either the existing systems were inadequate or they were not being properly used. For example, in Plant 2 a cover was usually, but not always, placed over the mold and ventilation duct by the workers. This measurably reduced air flow and capture of process emissions. Additionally, the thermodynamics of the process may have been such that there were significant emissions in spite of existing ventilation.

\section{CONCLUSIONS AND RECOMMENDATIONS}

The results of sampling during the May 2007, NIOSH in-depth survey indicate that $71 \%$ (17/24) of the personal breathing zone samples collected for airborne beryllium concentrations exceeded the NIOSH REL of $0.5 \mu \mathrm{g} / \mathrm{m}^{3}$ (the most restrictive OEL). Twenty-nine percent $(7 / 24)$ were above the less restrictive OSHA-PEL and ACGIH-TLV both of which are $2.0 \mu \mathrm{g} / \mathrm{m}^{3}$.

Additionally, one beryllium sample exceeded the maximum use concentration (MUC) for the respirator being used by the workers at this plant, and two others had concentrations that almost exceeded the MUC. The MUC for a respirator is defined as the OEL, in this case the NIOSHREL, multiplied by the assigned protection factor (APF) for that specific respirator. ${ }^{19}$ The halfface air-purifying respirators used by the workers at this facility have an APF of 10 . The calculated MUC using the less restrictive OSHA PEL $\left(2.0 \mu \mathrm{g} / \mathrm{m}^{3}\right)$ the MUC is $20.0 \mu \mathrm{g} / \mathrm{m}^{3}$ meaning that the respirator will protect the worker from airborne beryllium concentrations up to $20 \mu \mathrm{g} / \mathrm{m}^{3}$ or $5 \mu \mathrm{g} / \mathrm{m}^{3}$ when using the more protective NIOSH-REL.

When compared to the legally enforceable OSHA PEL, none of the airborne concentrations exceed the calculated MUC $\left(20 \mu \mathrm{g} / \mathrm{m}^{3}\right)$ and the highest concentration detected $\left(5.52 \mu \mathrm{g} / \mathrm{m}^{3}\right)$ is approximately $25 \%$ of calculated MUC. However, the highest concentration detected $(5.52$ $\left.\mu \mathrm{g} / \mathrm{m}^{3}\right)$ exceeds the MUC $\left(5.0 \mu \mathrm{g} / \mathrm{m}^{3}\right.$ - based on the NIOSH REL) for the type of respirators used 
at this facility. Additionally, two samples approached the MUC with concentrations of 4.72 $\mu \mathrm{g} / \mathrm{m}^{3}(94 \%)$ and $4.79 \mu \mathrm{g} / \mathrm{m}^{3}(96 \%)$. The remaining 21 of 24 samples were all $50 \%$ or less than the MUC. The jobs/areas where airbome beryllium concentrations approached or exceeded the MUC for the respirators used in this facility were the furnace operators and the grinder.

Controlling worker exposures to beryllium dust and fume can be accomplished through the use of engineering controls, work practices, administrative actions, and personal protective equipment (PPE). Engineering controls include such things as isolating the source and using ventilation systems to control dust and is the preferred method for controlling worker exposures. Administrative actions include limiting the worker's exposure time and providing showers. PPE includes wearing the proper respiratory protection and personal protective clothing. The respirators being used at the time of the NIOSH survey in May 2007, would afford adequate protection from airborne beryllium concentrations measured when compared against the legally enforceable OSHA PEL, however, they would not be when compared to the more protective NIOSH REL. Therefore, consideration should be given to upgrading the respiratory protection currently provided to the workers.

Since the time of the NIOSH survey (May 2007), the company has been conducting a comprehensive upgrade of entire ventilation system in the foundry areas of plant 1 and plant 2 to reduce airbome concentrations below the OELs. After the upgrade to the ventilation system is completed it is recommended that additional beryllium sampling be conducted to determine the effectiveness of the upgrades toward reducing airbome beryllium concentrations. Additionally, it is recommended that the grinding and fumace operations be given special attention due to the overexposures in those areas.

Some general recommendations for reducing airborme beryllium concentrations and controlling worker exposures to beryllium-containing dust and fume include:

- Only employees who have been cleared to work in beryllium designated areas should be allowed access to areas where beryllium is used.

- Employees should receive regular training on the proper handling of beryllium, as well as the hazards of beryllium exposure. Additionally, employees should receive periodic training on the use of all engineering controls associated with their operations to ensure their understanding of how the controis reduce the concentrations of airborne beryllium particles.

- The use of dry sweeping techniques in beryllium designated work areas should be discontinued, the use of HEPA-filtered vacuums to remove dust from floors and work surfaces is recommended.

- A written respiratory protection program should be implemented and should include: the training of employees; the selection, maintenance, and use of respirators; and monitoring of the program to ensure its ongoing effectiveness.

- Employees involved in the furnace melting and pouring operations should be provided face shields for protection from hot metals.

- The installation of a locker/change room equipped with lockers and showers is recommended. A shower room for exposed workers to shower and change from contaminated, company-provided work clothes into street clothes before leaving the 
facility reduces the potential for post-work exposure as well as the possibility of carrying contamination home.

Other guidelines for housekeeping in workplaces that use beryllium are available from several sources. In 1999, OSHA issued a Hazard Information Bulletin, Preventing Adverse Health Effects from Exposure to Beryllium on the Job (OSHA 1999). The web link to that document is provided below:

http://www.osha.gov/dts/hib/hib_data/hib19990902.html

There are several sources of information on engineering controls including the ACGIH Industrial Ventilation Manual. ${ }^{20}$ Another excellent source for materials on engineering controls and respiratory protection for foundry processes can be found at the British Health and Safety Executive website. Web links specific to a few of the processes at your facility are provided below:

http://www.hse.gov.uk/pubns/ (HSE Publication Web Link) http://www.hse.gov.uk/pubns/guidance/g406.pdf (New and existing engineering control systems)

http://wwww.hse.gov.uk/pubns/guidance/fd1.pdf (Fume: General ventilation) http://www.hse.gov.uk/pubns/guidance/fd2.pdf (Molten metal fume: Melting) http://www.hse.gov.uk/pubns/guidance/fd3.pdf (Molten metal fume: Pouring and casting) 
Beryllium Facility \#1 - Plant Diagram

\section{Copper/Beryllium Foundry and Machine Shop}

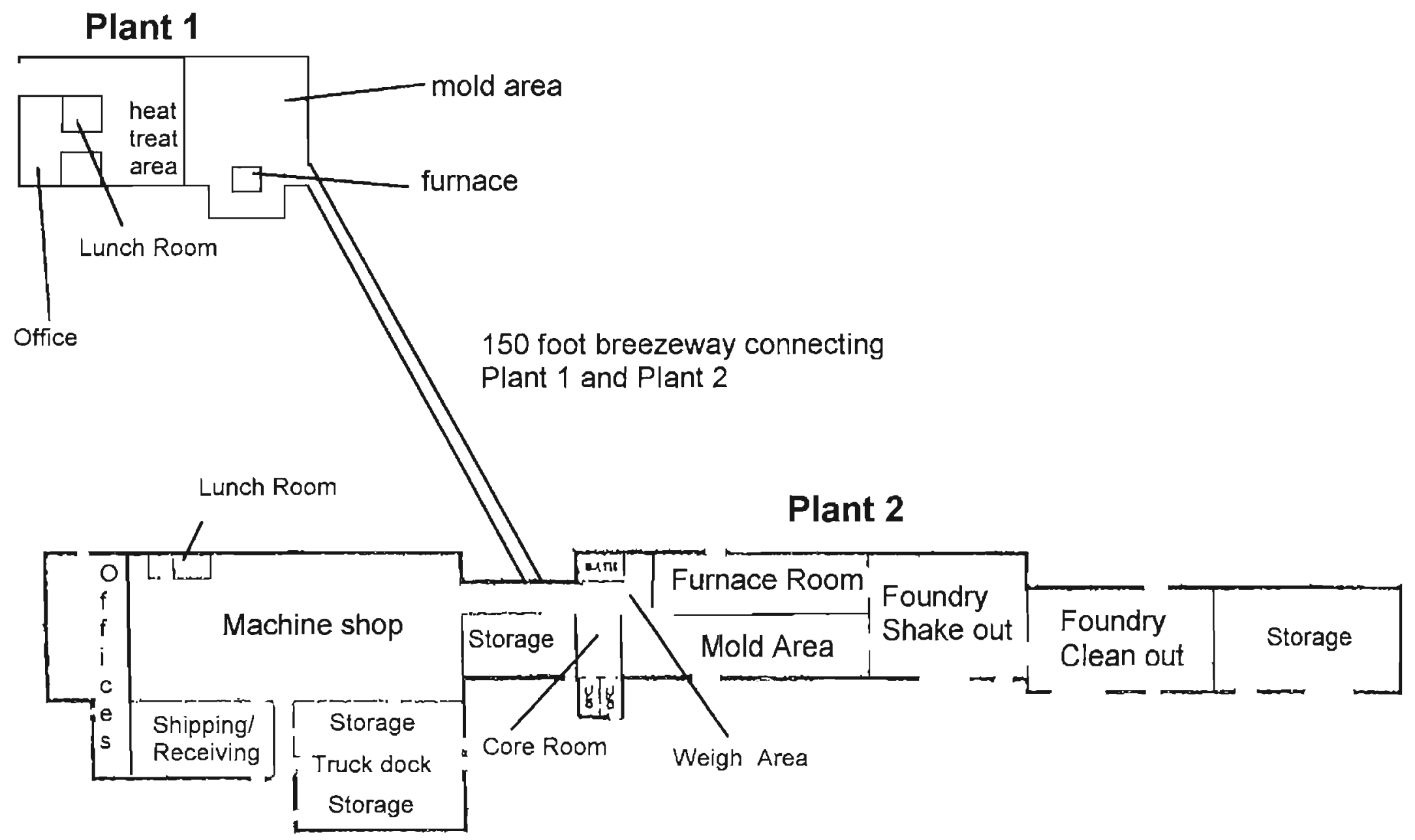


Table 1

Occupational Exposure Criteria for Metal/Elements

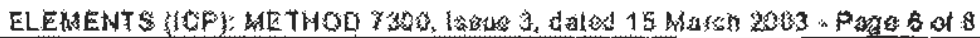

FAELE 2. EXPOSURE LIHITS, OAS RTECS

\begin{tabular}{|c|c|c|c|c|c|}
\hline 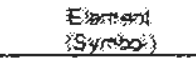 & CAS & ALES & DSHA & 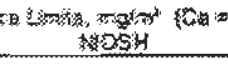 & 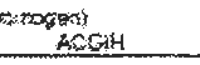 \\
\hline Exygx ingi & $74+204$ & Wh 3400000 & 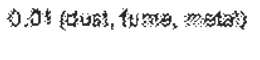 & 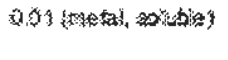 & 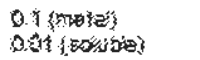 \\
\hline 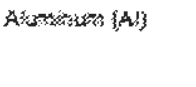 & 742004 & $5[0005010$ & 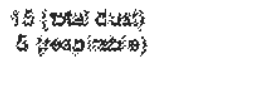 & 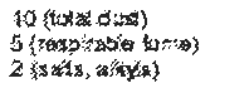 & 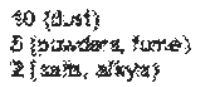 \\
\hline Ansoris $\{A\}$ & 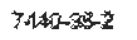 & $\cos 525000$ & $\tan$ & 9026 & 004,6 \\
\hline 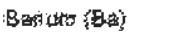 & 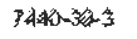 & 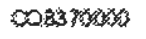 & $\sin$ & 5 & $O B$ \\
\hline 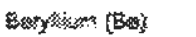 & 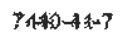 & 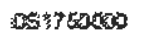 & $4072, c 0005$ & 40000.0 & $0, \cos 2 . \mathrm{ca}$ \\
\hline 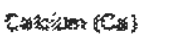 & 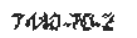 & $\rightarrow$ & 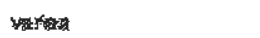 & vatis & शघहल्रि \\
\hline 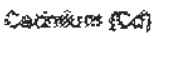 & $7(x+1)-10$ & 든 & $\Delta a$ & 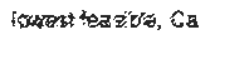 & 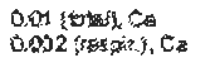 \\
\hline Cobers & $74+20-1 E-4$ & aratsonos & $a$ & 1. & O. \\
\hline Ctramitlan: \{t: $\}$ & $M(10-1 T+3$ & $\operatorname{sen} 2004$ & 0.5 & g & 05 \\
\hline Coppera $\{0$ to & 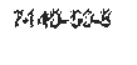 & Sts 525000 & 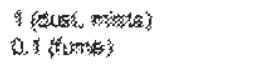 & 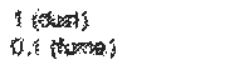 & 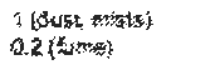 \\
\hline 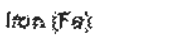 & 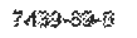 & $\times 100509$ & 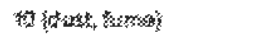 & 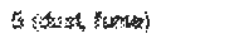 & 5 Hongis: \\
\hline Fordent & 740009 & TESOSOS & 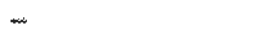 & $-\infty$ & - \\
\hline 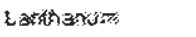 & $7459-4110$ & - & - & - & - \\
\hline Lath $\{2\}$ & $740-202$ & - & - & $\cdots$ & $m$ \\
\hline Nognasus onto & JAA & 04210000 & 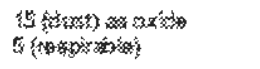 & 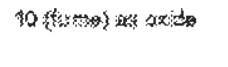 & 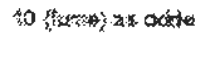 \\
\hline 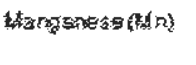 & $7+5066$ & 20001500 & $6 \mathrm{~s}$ & stes & 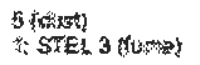 \\
\hline Holdom wa & 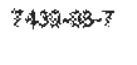 & $04+202039$ & 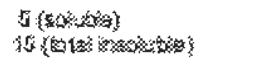 & 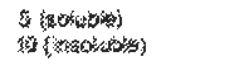 & 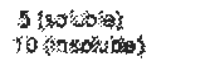 \\
\hline 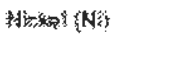 & 744020 & $\cos 5009$ & 1 & Q.915: E & 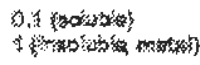 \\
\hline Fond & $7 \times 25+46$ & $F+350000 \%$ & 0.1 & $a i$ & 0.3 \\
\hline Losd & thato $22-5$ & QFF 300000 & 0.05 & $40 \%$ & nos \\
\hline 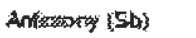 & 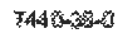 & $60402500 \%$ & 45 & 0.8 & 05 \\
\hline SFeran $\times 3 \times$ & $\pi 82+9-2$ & Estotosto & 52 & 0.2 & $0 z$ \\
\hline 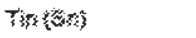 & $x \rightarrow 130-30-x$ & $x+3320,0$ & 2 & 2 & $z$ \\
\hline Shortign $(5)$ & $7+d a b 34$ & $\omega$ & $m$ & $\sim$ & - \\
\hline 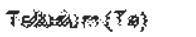 & 3舟4 & thesestoto & ตั. & D. \{ & 0.5 \\
\hline 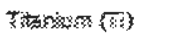 & $3484-32-4$ & XR: 10000 & - & - & 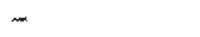 \\
\hline 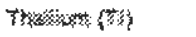 & $7439-280$ & $x 3342000$ & 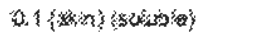 & 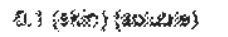 & 0,4 (sing \\
\hline 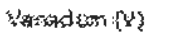 & ZAd $0 x a x-3$ & Y & 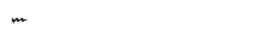 & 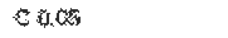 & 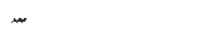 \\
\hline Turg: & Tata-20-7 & - & 5 & 30 & 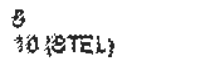 \\
\hline 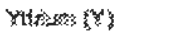 & $x+10-25-5$ & 27800000 & 8 & Hes & * \\
\hline 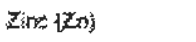 & 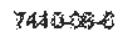 & $2 \cos 00 \sin$ & $\omega$ & دا & $\sim$ \\
\hline Finengan $\{Z\}$ & TQA $0107-7$ & 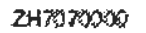 & 5 & 至 STER $M$ & 혹 s7돈 \\
\hline
\end{tabular}

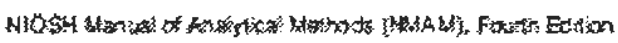


Table 2

BERYLLIUM FACILITY \#1 - Copper/Beryllium Foundry

Personal Breathing Zone and Area Alr Sample Results for Beryllium (Be) and Copper (Cu)

\begin{tabular}{|c|c|c|c|c|c|c|}
\hline Sample Number & Job Description/Work Location & Date & Time(min.) & Sample Type & $B e\left(\mu \mathrm{g} / \mathrm{m}^{3}\right)$ & $\mathrm{Cu}\left(\mu \mathrm{g} / \mathrm{m}^{3}\right)$ \\
\hline SMTF-7 & Saw Operator/Plant 1 & $5 / 15 / 2007$ & 563 & Personal $(P)$ & $2.54 *$ & $168(\mathrm{D})$ \\
\hline SMTF-8 & Mold Maker/Plant 1 & $5 / 15 / 2007$ & 585 & $\mathrm{P}$ & 0.24 & $5.44(\mathrm{D})$ \\
\hline SMTF-9 & Core Maker/Plant 1 & $5 / 15 / 2007$ & 588 & $P$ & 0.57 & $9.36(\mathrm{D})$ \\
\hline SMTF-10 & Furnace Operator/Plant 2 & $5 / 15 / 2007$ & 558 & $\mathrm{P}$ & $4.72^{*}$ & $36.9(\mathrm{~F})$ \\
\hline SMTF-11 & Furnace Operator/Plant 1 & $5 / 15 / 2007$ & 564 & $\mathrm{P}$ & $5.52^{*}$ & $50.4(F)$ \\
\hline SMTF-12 & Supervisor/Plant 2 & $5 / 15 / 2007$ & 620 & $\mathrm{P}$ & 1.59 & $23.1(F)$ \\
\hline SMTF-13 & Mold Clean-out/Plant 1 & $5 / 15 / 2007$ & 296 & $\mathrm{P}$ & 0.81 & $52.1(\mathrm{D})$ \\
\hline SMTF-14 & Metal Weigh-up/Plant 1 & $5 / 15 / 2007$ & 582 & $\mathrm{P}$ & 1.14 & $55.8(\mathrm{D})$ \\
\hline SMTF-15 & Core-bench Specialist/Lab & $5 / 15 / 2007$ & 583 & $\mathrm{P}$ & 0.81 & $13.2(D)$ \\
\hline SMTF-16 & Area near MC-90/Machine Shop & $5 / 15 / 2007$ & 397 & Area (A) & 0.07 & (3.53) (D) \\
\hline SMTF-17 & Area near NC-70/Machine Shop & $5 / 15 / 2007$ & 395 & A & 0.08 & $(4.34)(D)$ \\
\hline SMTF-18 & Supervisor/Plant 2 & $5 / 15 / 2007$ & 464 & $\mathrm{P}$ & 1.60 & $25.5(\mathrm{~F})$ \\
\hline SMTF-19 & Supervisor/Machine Shop & $5 / 15 / 2007$ & 501 & $\mathrm{P}$ & $2.17^{k}$ & $44.2(D)$ \\
\hline SMTF-20 & Area near NC-50/Machine Shop & $5 / 15 / 2007$ & 394 & A & 0.06 & $(3.72)(D)$ \\
\hline SMWF-10 & Mold Clean-out/Plant 1 & $5 / 16 / 2007$ & 540 & $P$ & $2.29^{*}$ & $36.3(D)$ \\
\hline SMWF-11 & Mold Maker/Plant 1 & $5 / 16 / 2007$ & 568 & $\mathrm{P}$ & 0.25 & $(4.66)(D)$ \\
\hline SMWF-12 & Mold Maker/Plant 1 & $5 / 16 / 2007$ & 567 & $\mathrm{P}$ & 0.33 & $6.95(D)$ \\
\hline SMWF-13 & Core Maker/Plant 1 & $5 / 16 / 2007$ & 589 & $\mathrm{P}$ & 0.43 & $8.17(\mathrm{D})$ \\
\hline SMWF-14 & Mold Remover/Plant 1 & $5 / 16 / 2007$ & 587 & $\mathrm{P}$ & 1.30 & $31.3(D)$ \\
\hline SMWF-15 & Grinder/Plant 1 & $5 / 16 / 2007$ & 586 & $\mathrm{P}$ & $4.79^{*}$ & $146(\mathrm{D})$ \\
\hline SMWF-16 & Mold Maker/Plant 1 & $5 / 16 / 2007$ & 585 & $\mathrm{P}$ & 0.46 & $17.4(\mathrm{D})$ \\
\hline SMWF-17 & Furnace Operator/Plant 2 & $5 / 16 / 2007$ & 528 & $\mathbf{P}$ & 1.16 & $42.8(\mathrm{~F})$ \\
\hline SMWF-18 & Mold Pourer/Plant 2 & $5 / 16 / 2007$ & 487 & $\mathbf{P}$ & 1.40 & $43.6(\mathrm{~F})$ \\
\hline SMWF-19 & Mold Pourer/Plant 2 & $5 / 16 / 2007$ & 520 & $\mathbf{P}$ & $2.04 *$ & $34.9(F)$ \\
\hline SMWF-21 & Machinist NC-90/Machine Shop & $5 / 16 / 2007$ & 441 & $\mathbf{P}$ & 0.13 & $6.14(\mathrm{D})$ \\
\hline SMWF-22 & Machinist NC-60/Machine Shop & $5 / 16 / 2007$ & 357 & $\mathbf{P}$ & 0.27 & $11.1(\mathrm{D})$ \\
\hline SMWF-23 & Machinist NC 50 \& 80/Machine Shop & $5 / 16 / 2007$ & 438 & $\mathbf{P}$ & 0.15 & $6.04(D)$ \\
\hline & Evaluation Criteria & \multicolumn{3}{|c|}{$\begin{array}{c}\text { NIOSH REL } \\
\text { " } \\
\text { OSHA PEL } \\
\end{array}$} & $\begin{array}{l}0.5 \\
2.0 \\
\end{array}$ & $\begin{array}{l}\text { Fume }(F)=100 \\
\text { Dust }(D)=1000 \\
\text { Same as NIOSH }\end{array}$ \\
\hline
\end{tabular}

$\mu \mathrm{g} / \mathrm{m}^{3}=$ micrograms per cubic meter of air

Bolded values exceed the NIOSH REL for beryllium

* - sample also exceeded the OSHA PEL for beryllium 
Table 3

FACILITY \#1 - Copper/Beryllium Foundry and Machine Shop

Ghost Wipes (G) and Palintest (P) Surface Wipe Sample Results for Beryllium (Be), Cadmium (Cd), Copper (Cu), Lead (Pb), aud Nickel (Ni)

\begin{tabular}{|c|c|c|c|c|c|c|c|c|}
\hline $\begin{array}{l}\text { Sample } \\
\text { Number }\end{array}$ & Sample Location & $\begin{array}{c}\text { Sample } \\
\text { Date }\end{array}$ & $\begin{array}{c}\text { Sample } \\
\text { Type }\end{array}$ & $\begin{array}{c}\text { Be } \\
\text { Conc. }^{\star}\end{array}$ & $\begin{array}{c}\text { Cd } \\
\text { Conc.* }\end{array}$ & $\begin{array}{c}\text { Cu } \\
\text { Conc.* }\end{array}$ & $\begin{array}{c}\text { Pb } \\
\text { Conc.* }\end{array}$ & Ni Conc.* \\
\hline SMMW-1 & Table top in IH set-up room before survey & $5 / 14 / 2007$ & $\mathrm{G}$ & 5.9 & $<0.10$ & 100 & 0.61 & $<3.0$ \\
\hline SMMW-2 & On top of the refrigerator in the break room & $5 / 14 / 2007$ & $\mathrm{G}$ & 21 & $<0.10$ & 540 & 1.80 & 6.1 \\
\hline SMTW-1 & On top of exhaust out of $\# 2$ kiln in plant 2 & $5 / 15 / 2007$ & $\mathrm{G}$ & 130 & 0.45 & 1100 & 3.00 & 15 \\
\hline SMTW-2 & On top of exhaust out of \#2 kiln in plant 2 & $5 / 15 / 2007$ & $\mathrm{P}$ & 120 & na & na & na & na \\
\hline SMTW-3 & On to of foundry equipment - hot shot (control button) & $5 / 15 / 2007$ & $\mathrm{G}$ & 210 & 0.55 & 5400 & 16.0 & 63 \\
\hline SMTW-4 & On top of cart in metal weighing area & $5 / 15 / 2007$ & $\mathrm{G}$ & 100 & 0.50 & 4100 & 11.0 & 74 \\
\hline SMTW-5 & On top of desk in kiln area in plant 1 (photo 3 ) & $5 / 15 / 2007$ & $\mathrm{G}$ & 92 & $<0.10$ & 1400 & 3.40 & 24 \\
\hline SMTW-6 & On top of desk in kiln area in plant 1 (photo 3 ) & $5 / 15 / 2007$ & $\mathrm{P}$ & 48 & na & na & na & na \\
\hline SMTW-7 & On top of table in front of core maker M50XL & $5 / 15 / 2007$ & $\mathrm{G}$ & 1.3 & 0.33 & 35 & 0.44 & $<3.0$ \\
\hline SMTW-8 & On top of core removal and clean out table & $5 / 15 / 2007$ & G & 11 & $<0.10$ & 840 & 2.00 & 6.7 \\
\hline SMTW-9 & On top of table of cut-off saw in plant 1 & $5 / 15 / 2007$ & $\mathrm{G}$ & 26 & $<0.10$ & 1900 & 4.10 & 15 \\
\hline SMTW-10 & On to of table in core assembly area & $5 / 15 / 2007$ & $\mathrm{G}$ & 1.4 & $<0.10$ & 16 & $<0.30$ & $<3.0$ \\
\hline SMTW-11 & On to of table in core assembly area & $5 / 15 / 2007$ & $\mathrm{P}$ & 1.0 & na & na & na & na \\
\hline SMWW-1 & On top of exhaust out of \#2 kiln in plant 2 & $5 / 16 / 2007$ & $\mathrm{P}$ & 90 & na & na & na & na \\
\hline SMWW-2 & On top of exhaust out of \#2 kiln in plant 2 & $5 / 16 / 2007$ & $\mathrm{G}$ & 74 & $<0.10$ & 980 & 2.30 & 9.3 \\
\hline SMWW-3 & On top of a transformer behind NC-20 in machine shop & $5 / 1620 / 07$ & $\mathrm{G}$ & 4.2 & $<0.10$ & 360 & 1.40 & 3.6 \\
\hline SMWW-4 & On top of a small scale in metal weighing area & $5 / 16 / 2007$ & $\mathrm{G}$ & 25 & 0.14 & 1600 & 21.0 & 37 \\
\hline SMWW-5 & On a cart in the kiln area of plant 1 & $5 / 16 / 2007$ & $\mathrm{G}$ & 1200 & 1.20 & 11000 & 25.0 & 220 \\
\hline SMWW-6 & On top of control box C-1 behind a kiln in plant 1 & $5 / 16 / 2007$ & $\mathrm{G}$ & 1100 & 0.30 & 9900 & 21.0 & 150 \\
\hline SMWW-7 & On top of control box $\mathrm{C}-1$ behind a kiln in plant 1 & $5 / 16 / 2007$ & $P$ & 1010 & na & na & na & na \\
\hline SMWW-8 & Table top under vent 4 control panel behind kiln in plant 1 & $5 / 16 / 2007$ & $\mathrm{G}$ & 110 & 0.64 & 2800 & 8.20 & 38 \\
\hline SMWW-9 & Table top near J-M-2 mold maker mixer 1 & $5 / 16 / 2007$ & $\mathrm{G}$ & 55 & 0.44 & 1900 & 6.80 & 22 \\
\hline SMWW-10 & On top of belt guard on grinder in plant 1 & $5 / 16 / 2007$ & $\mathrm{G}$ & 210 & $<0.10$ & 9000 & 16.0 & 49 \\
\hline SMWW-11 & On top of belt guard on grinder in plant 1 & $5 / 16 / 2007$ & $\mathrm{P}$ & 64 & na & na & na & na \\
\hline SMWW-12 & TSI aerodynamic particle sizer top in grinding area of plant 1 & $5 / 16 / 2007$ & $\mathrm{G}$ & 3.2 & $<0.10$ & 110 & 0.60 & $<3.0$ \\
\hline SMWW-13 & TSI aerodynamic particle sizer top in grinding area of plant 1 & $5 / 16 / 2007$ & $\mathrm{P}$ & 1.1 & na & na & na & na \\
\hline SMWW-14 & Office desk top & $5 / 16 / 2007$ & $\mathrm{G}$ & 1.7 & $<0.10$ & 40 & $<0.30$ & $<3.0$ \\
\hline SMWW-15 & Office desk top & $5 / 16 / 2007$ & $\mathrm{P}$ & 1.1 & na & na & na & na \\
\hline
\end{tabular}

${ }^{\star} \mathrm{\mu g} / 100 \mathrm{~cm}^{2}=$ micrograms per 100 square centimeters of wiped surface

$\mathrm{G}=$ Ghost wipes

$\mathrm{P}=$ Palintest wipes, samples analyzed only for $\mathrm{Be}$

na $=$ sample result not available, sample analyzed only for $\mathrm{Be}$ 
TABLE 4

FACILITY \#1 - Copper/Beryllium Foundry and Machine Shop

Personal Breathing Zone (P) and Area (A) Sioutas Cascade Size-Selective Impactor Air Sample Results for Beryllium (Be) and Copper $(\mathrm{Cu})$ in micrograms per cubic meter of air $\left(\mu \mathrm{g} / \mathrm{m}^{3}\right)$

\begin{tabular}{|c|c|c|c|c|c|c|c|}
\hline $\begin{array}{l}\text { Semple } \\
\text { Number }\end{array}$ & Sample Description & $\begin{array}{c}\text { Sample } \\
\text { Type }\end{array}$ & $\begin{array}{c}\text { Sample } \\
\text { Date }\end{array}$ & $\begin{array}{l}\text { Sample } \\
\text { Time } \\
\text { (min.) }\end{array}$ & $\begin{array}{c}\text { Particle } \\
\text { Size } \\
(\mu \mathrm{m})\end{array}$ & $\begin{array}{c}\text { Be } \\
\text { Conc. } \\
\left(\mu g / \mathrm{m}^{3}\right)\end{array}$ & $\begin{array}{c}\text { Cu } \\
\text { Conc. } \\
\left(\mu \mathrm{g} / \mathrm{m}^{3}\right)\end{array}$ \\
\hline SMTF-1A & Fumace Operator/Plant 2 & P-fume & $5 / 15 / 2007$ & 561 & 2.5 & 0.90 & 28.1 \\
\hline SMTF-1B & Furnace Operator/Plant 2 & P-fume & $5 / 15 / 2007$ & 561 & 1.0 & 0.08 & 2.21 \\
\hline SMTF-1C & Furnace Operator/Plant 2 & P-fume & $5 / 15 / 2007$ & 561 & 0.50 & 0.01 & $<0.16$ \\
\hline SMTF-1D & Furnace Operator/Plant 2 & P-fume & $5 / 15 / 2007$ & 561 & 0.25 & 0.02 & $<0.16$ \\
\hline SMTF-1E & Furnace Operator/Plant 2 & P-fume & $5 / 15 / 2007$ & 561 & $<0.25$ & 0.02 & $<0.16$ \\
\hline SMTF-1 & Furnace Operator/Plant 2 & P-fume & $5 / 15 / 2007$ & & Total & 1.03 & 30.3 \\
\hline SMTF-2A & Cleanout/Plant 1 & P-dust & $5 / 15 / 2007$ & 565 & 2.5 & 1.23 & 15.6 \\
\hline SMTF-2B & Cleanout/Plant 1 & P-dust & $5 / 15 / 2007$ & 565 & 1.0 & 0.39 & 4.32 \\
\hline SMTF-2C & Cleanout/Plant 1 & P-dust & $5 / 15 / 2007$ & 565 & 0.50 & 0.12 & 0.88 \\
\hline SMTF-2D & Cleanout/Plant 1 & P-dust & $5 / 15 / 2007$ & 565 & 0.25 & 0.13 & 0.33 \\
\hline SMTF-2E & Cleanout/Plant 1 & P-dust & $5 / 15 / 2007$ & 565 & $<0.25$ & 0.14 & 1.40 \\
\hline SMTF-2 & Cleanout/Plant 1 & P-dust & $5 / 15 / 2007$ & & Total & 2.01 & 22.6 \\
\hline SMTF-3A & Grinder/Plant 1 & P-dust & $5 / 15 / 2007$ & 334 & 2.5 & 0.73 & 56.7 \\
\hline SMTF-3B & Grinder/Plant 1 & P-dust & $5 / 15 / 2007$ & 334 & 1.0 & 0.01 & 0.32 \\
\hline SMTF-3C & Grinder/Plant 1 & P-dust & $5 / 15 / 2007$ & 334 & 0.50 & $<0.01$ & $<0.27$ \\
\hline SMTF-3D & Grinder/Plant 1 & P-dust & $5 / 15 / 2007$ & 334 & 0.25 & $<0.01$ & $<0.27$ \\
\hline SMTF-3E & Grinder/Plant 1 & P-dust & $5 / 15 / 2007$ & 334 & $<0.25$ & $<0.01$ & $<0.27$ \\
\hline SMTF-3 & Grinder/Plant 1 & P-dust & $5 / 15 / 2007$ & & Total & 0.74 & 57.1 \\
\hline SMTF-4A & Furnace Room Plant 2 & P-fume & $5 / 15 / 2007$ & 568 & $2 . \overline{5}$ & 0.61 & 24.2 \\
\hline SMTF-4B & Furnace Room Plant 2 & P-fume & $5 / 15 / 2007$ & 568 & 1.0 & 0.20 & 8.88 \\
\hline SMTF-4C & Fumace Poom Plant 2 & P-fume & $5 / 15 / 2007$ & 568 & 0.50 & 0.07 & 3.03 \\
\hline SMTF-4D & Furnace Room Plant 2 & P-fume & $5 / 15 / 2007$ & 568 & 0.25 & 0.03 & 0.07 \\
\hline SMTF-4E & Fumace Room Plant 2 & P-fume & $5 / 15 / 2007$ & 568 & $<0.25$ & 0.07 & 1.96 \\
\hline SMTF-4 & Fumace Room Plant 2 & P-fume & $5 / 15 / 2007$ & & Total & 0.98 & 38.2 \\
\hline
\end{tabular}


TABLE 4-continued

FACILITY \#1 - Copper/Beryllium Foundry and Machine Shop

Personal Breathing Zone (P) and Area (A) Sioutas Cascade Size-Selective Impactor Air Sample

Results for Beryllium (Be) and Copper $(\mathrm{Cu})$ in micrograms per cubic meter of air $\left(\mu \mathrm{g} / \mathrm{m}^{3}\right)$

\begin{tabular}{|c|c|c|c|c|c|c|c|}
\hline $\begin{array}{l}\text { Sample } \\
\text { Number }\end{array}$ & Sample Description & $\begin{array}{c}\text { Sample } \\
\text { Type }\end{array}$ & $\begin{array}{c}\text { Sample } \\
\text { Date }\end{array}$ & $\begin{array}{c}\text { Sample } \\
\text { Time } \\
\text { (min.) }\end{array}$ & $\begin{array}{c}\text { Particle } \\
\text { Size } \\
\text { (pm) }\end{array}$ & $\begin{array}{c}\text { Be } \\
\text { Conc. } \\
\left(\mu \mathrm{g} / \mathrm{m}^{3}\right)\end{array}$ & $\begin{array}{c}\text { Cu } \\
\text { Conc. } \\
\left(\mu g / \mathrm{m}^{3}\right)\end{array}$ \\
\hline SMTF-5A & Core Maker & P-dust & $5 / 15 / 2007$ & 585 & 2.5 & 0.22 & 4.90 \\
\hline SMTF-5B & Core Maker & P-dust & $5 / 15 / 2007$ & 585 & 1.0 & 0.04 & 0.78 \\
\hline SMTF-5C & Core Maker & P-dust & $5 / 15 / 2007$ & 585 & 0.50 & 0.01 & $<0.16$ \\
\hline SMTF-5D & Core Maker & P-dust & $5 / 15 / 2007$ & 585 & 0.25 & $<0.004$ & $<0.16$ \\
\hline SMTF-5E & Core Maker & P-dust & $5 / 15 / 2007$ & 585 & $<0.25$ & $<0.004$ & $<0.16$ \\
\hline SMTF-5 & Core Maker & P-dust & $5 / 15 / 2007$ & & Total & 0.27 & 5.69 \\
\hline SMTF-6A & Mold Shop & P-dust & $5 / 15 / 2007$ & 553 & 2.5 & 0.16 & 4.60 \\
\hline SMTF-6B & Mold Shop & P-dust & $5 / 15 / 2007$ & 553 & 1.0 & 0.05 & 1.07 \\
\hline SMTF-6C & Mold Shop & P-dust & $5 / 15 / 2007$ & 553 & 0.50 & 0.01 & $<0.17$ \\
\hline SMTF-6D & Mold Shop & P-dust & $5 / 15 / 2007$ & 553 & 0.25 & $<0.004$ & $<0.17$ \\
\hline SMTF-6E & Mold Shop & P-dust & $5 / 15 / 2007$ & 553 & $<0.25$ & 0.004 & $<0.17$ \\
\hline SMTF-6 & Mold Shop & P-dust & $5 / 15 / 2007$ & & Total & 0.22 & 5.67 \\
\hline SMWF-1A & Mold Shop & P-dust & $5 / 16 / 2007$ & 599 & 2.5 & 0.12 & 3.12 \\
\hline SMWF-1B & Mold Shop & P-dust & $5 / 16 / 2007$ & 599 & 1.0 & 0.03 & 0.62 \\
\hline SMWF-1C & Mold Shop & P-dust & $5 / 16 / 2007$ & 599 & 0.50 & 0.01 & $<0.16$ \\
\hline SMWF-1D & Mold Shop & P-dust & $5 / 16 / 2007$ & 599 & 0.25 & $<0.004$ & $<0.16$ \\
\hline SMWF-1E & Mold Shop & P-dust & $5 / 16 / 2007$ & 599 & $<0.25$ & 0.01 & 0.20 \\
\hline SMWF-1 & Mold Shop & P-dust & $5 / 16 / 2007$ & & Total & 0.17 & 3.94 \\
\hline SMWF-2A & Cleanout Plant 1 & P-dust & $5 / 16 / 2007$ & 545 & 2.5 & 1.08 & 45.7 \\
\hline SMWF-2B & Cleanout Plant 1 & P-dust & $5 / 16 / 2007$ & 545 & 1.0 & 0.29 & 11.4 \\
\hline SMWF-2C & Cleanout Plant 1 & P-dust & $5 / 16 / 2007$ & 545 & 0.50 & 0.06 & 2.04 \\
\hline SMWF-2D & Cleanout Plant 1 & P-dust & $5 / 16 / 2007$ & 545 & 0.25 & 0.02 & 0.46 \\
\hline SMWF-2E & Cleanout Plant 1 & P-dust & $5 / 16 / 2007$ & 545 & $<0.25$ & 0.02 & 0.77 \\
\hline SMWF-2 & Cleanout Plant 1 & P-dust & $5 / 16 / 2007$ & & Total & 1.48 & 60.4 \\
\hline
\end{tabular}


TABLE 4 - continued

FACILITY \#1 - Copper/Beryllinm Foundry and Machine Shop

Personal Breathing Zone (P) and Area (A) Sioutas Caseade Size-Selective Impactor Air Sample Results for Beryllium (Be) and Copper (Cu) in micrograms per cubic meter of air $\left(\mu \mathrm{g} / \mathrm{m}^{3}\right)$

\begin{tabular}{|c|c|c|c|c|c|c|c|}
\hline $\begin{array}{l}\text { Sample } \\
\text { Number }\end{array}$ & Sample Description & $\begin{array}{c}\text { Sample } \\
\text { Type }\end{array}$ & $\begin{array}{c}\text { Sample } \\
\text { Date }\end{array}$ & $\begin{array}{c}\text { Sample } \\
\text { Time } \\
\text { (min.) }\end{array}$ & $\begin{array}{c}\text { Particle } \\
\text { Size } \\
\text { (ym) }\end{array}$ & $\begin{array}{c}\text { Be Conc. } \\
\left(\mu \mathrm{g} / \mathrm{m}^{3}\right)\end{array}$ & $\begin{array}{c}\text { Cu } \\
\text { Cone. } \\
\left(\mu g / m^{3}\right)\end{array}$ \\
\hline SMWF-3A & Furnace Operator Plant 1 & P-fume & $5 / 16 / 2007$ & 586 & 2.5 & 1.60 & 22.9 \\
\hline SMWF-3B & Fumace Operator Plant 1 & P-fume & $5 / 16 / 2007$ & 586 & 1.0 & 0.37 & 4.16 \\
\hline SMWF-3C & Furnace Operator Plant 1 & P-fume & $5 / 16 / 2007$ & 586 & 0.50 & 0.11 & 1.08 \\
\hline SMWF-3D & Furnace Operator Plant 1 & P-fume & $5 / 16 / 2007$ & 586 & 0.25 & 0.11 & 0.69 \\
\hline SMWF-3E & Furnace Operator Plant 1 & P-fume & $5 / 16 / 2007$ & 586 & $<0.25$ & 0.06 & 0.92 \\
\hline SMWF-3 & Furnace Operator Plant 1 & P-fume & $5 / 16 / 2007$ & & Total & 2.26 & 29.74 \\
\hline SMWF-4A & Weigh-Out Plant 1 & P-dust & $5 / 16 / 2007$ & 589 & 2.5 & 0.87 & 38.6 \\
\hline SMWF-4B & Weigh-Out Plant 1 & P-dust & $5 / 16 / 2007$ & 589 & 1.0 & 0.22 & 8.53 \\
\hline SMWF-4C & Weigh-Out Plant 1 & P-dust & $5 / 16 / 2007$ & 589 & 0.50 & 0.06 & 2.44 \\
\hline SMWF-4D & Weigh-Out Plant 1 & P-dust & $5 / 16 / 2007$ & 589 & 0.25 & 0.02 & 0.41 \\
\hline SMWF-4E & Weigh-Out Plant 1 & P-dust & $5 / 16 / 2007$ & 589 & $<0.25$ & 0.02 & 0.65 \\
\hline SMWF-4 & Weigh-Out Plant 1 & P-dust & $5 / 16 / 2007$ & & Total & 1.20 & 50.6 \\
\hline SMWF-5A & Cut-off Plant 1 & P-dust & $5 / 16 / 2007$ & 538 & 2.5 & 6.05 & 230 \\
\hline SMWF-5B & Cut-off Plant 1 & P-dust & $5 / 16 / 2007$ & 538 & 1.0 & 1.79 & 62.6 \\
\hline SMWF-5C & Cut-off Plant 1 & P-dust & $5 / 16 / 2007$ & 538 & 0.50 & 0.65 & 23.0 \\
\hline SMWF-5D & Cut-off Plant 1 & P-dust & $5 / 16 / 2007$ & 538 & 0.25 & 0.23 & 7.51 \\
\hline SMWF-5E & Cut-off Plant 1 & P-dust & $5 / 16 / 2007$ & 538 & $<0.25$ & 0.25 & 8.77 \\
\hline SMWF-5 & Cut-off Plant 1 & P-dust & $5 / 16 / 2007$ & & Total & 8.97 & $33 \uparrow$ \\
\hline SMWF-6A & Supervisor Plant 2 & P-fume & $5 / 16 / 2007$ & 585 & 2.5 & 0.33 & 11.3 \\
\hline SMWF-6B & Supervisor Plant 2 & P-fume & $5 / 16 / 2007$ & 585 & 1.0 & 0.10 & 3.27 \\
\hline SMWF-6C & Supervisor Plant 2 & P-fume & $5 / 16 / 2007$ & 585 & 0.50 & 0.03 & 0.83 \\
\hline SMWF-6D & Supervisor Plant 2 & P-fume & $5 / 16 / 2007$ & 585 & 0.25 & 0.03 & 0.71 \\
\hline SMWF-6E & Supervisor Plant 2 & P-fume & $5 / 16 / 2007$ & 585 & $<0.25$ & 0.05 & 2.69 \\
\hline SMWF-6 & Supervisor Plant 2 & P-fume & $5 / 16 / 2007$ & & Total & 0.54 & 18.8 \\
\hline
\end{tabular}


TABLE 4- continued

FACILITY \#1 - Copper/Beryllium Foundry and Machine Shop

Personal Breathing Zone (P) and Area (A) Sioutas Cascade Size-Selective Impactor Air Sample Results for Beryllium (Be) and Copper (Cu) in micrograms per cubic meter of air $\left(\mu \mathrm{g} / \mathrm{m}^{3}\right)$

\begin{tabular}{|c|c|c|c|c|c|c|c|}
\hline $\begin{array}{l}\text { Sample } \\
\text { Number }\end{array}$ & Sample Descrlption & $\begin{array}{c}\text { Sample } \\
\text { Type }\end{array}$ & $\begin{array}{c}\text { Sample } \\
\text { Date }\end{array}$ & $\begin{array}{c}\text { Sample } \\
\text { Time } \\
\text { (min-) }\end{array}$ & $\begin{array}{c}\text { Particle } \\
\text { Size } \\
\langle\mu \mathrm{m}\rangle\end{array}$ & $\begin{array}{c}\text { Be } \\
\text { Conc } \\
\left(\mu \mathrm{g} / \mathrm{m}^{3}\right)\end{array}$ & $\begin{array}{c}\mathrm{Cu} \\
\mathrm{Conc} \\
\left(\mu \mathrm{g} / \mathrm{m}^{3}\right)\end{array}$ \\
\hline SMWF-7A & Furnace Operator Plant 2 & P-fume & $5 / 16 / 2007$ & 528 & 2.5 & 2.57 & 23.6 \\
\hline SMWF-7B & Furnace Operator Plant 2 & P-fume & $5 / 16 / 2007$ & 528 & 1.0 & 0.56 & 4.93 \\
\hline SMWF-7C & Furnace Operator Plant 2 & P-fume & $5 / 16 / 2007$ & 528 & 0.50 & 0.16 & 0.99 \\
\hline SMWF-7D & Furnace Operator Plant 2 & P-fume & $5 / 16 / 2007$ & 528 & 0.25 & 0.12 & 0.73 \\
\hline SMWF-7E & Furnace Operator Plant 2 & P-fume & $5 / 16 / 2007$ & 528 & $<0.25$ & 0.26 & 1.52 \\
\hline SMWF-7 & Furnace Operator Plant 2 & P-fume & $5 / 16 / 2007$ & & Total & 3.67 & 31.7 \\
\hline SMWF-8A & NC-20 Machine Shop & A-dust & $5 / 16 / 2007$ & 435 & 2.5 & 0.04 & 1.74 \\
\hline SMWF-8B & NC-20 Machine Shop & A-dust & $5 / 16 / 2007$ & 435 & 1.0 & 0.01 & 0.29 \\
\hline SMWF-8C & NC-20 Machine Shop & A-dust & $5 / 16 / 2007$ & 435 & 0.50 & $<0.01$ & $<0.21$ \\
\hline SMWF-8D & NC-20 Machine Shop & A-dust & $5 / 16 / 2007$ & 435 & 0.25 & $<0.01$ & $<0.21$ \\
\hline SMWF-8E & NC-20 Machine Shop & A-dust & $5 / 16 / 2007$ & 435 & $<0.25$ & $<0.01$ & $<0.21$ \\
\hline SMWF-8 & NC-20 Machine Shop & A-dust & $5 / 16 / 2007$ & & Total & 0.05 & 2.03 \\
\hline
\end{tabular}


Table 5

FACILITY \#1 - Copper/Beryllium Foundry and Machine Shop Summary of the APS Sample Data

\begin{tabular}{|c|c|c|c|c|}
\hline Sample location & Date & Geometric mean $(\mu \mathrm{m})$ & Mode $(\mu m)$ & $\begin{array}{c}\text { Particle number Concentration at } \\
\text { mode size }\left(\sharp / \mathrm{cm}^{3}\right)\end{array}$ \\
\hline A - furnace, plant 1 & $5 / 15 / 2007$ & 0.759 & 0.673 & 424.4 \\
\hline B - furnace, plant 2 & $5 / 15 / 2007$ & 0.789 & 0.626 & 346.1 \\
\hline C - cutting/grinding, plant 2 & $5 / 15 / 2007$ & $0 . \overline{885}$ & 0.673 & 242.7 \\
\hline A - furnace, plant 1 & $5 / 16 / 2007$ & 0.793 & 0.835 & 364.7 \\
\hline B - furnace, plant 2 & $5 / 16 / 2007$ & 0.756 & 0.626 & 147.0 \\
\hline C - cutting/grinding, plant2 & $5 / 16 / 2007$ & 0.939 & 0.626 & 127.8 \\
\hline Office & $5 / 16 / 2007$ & 0.842 & 0.626 & 50.2 \\
\hline
\end{tabular}

\section{Figure 1}

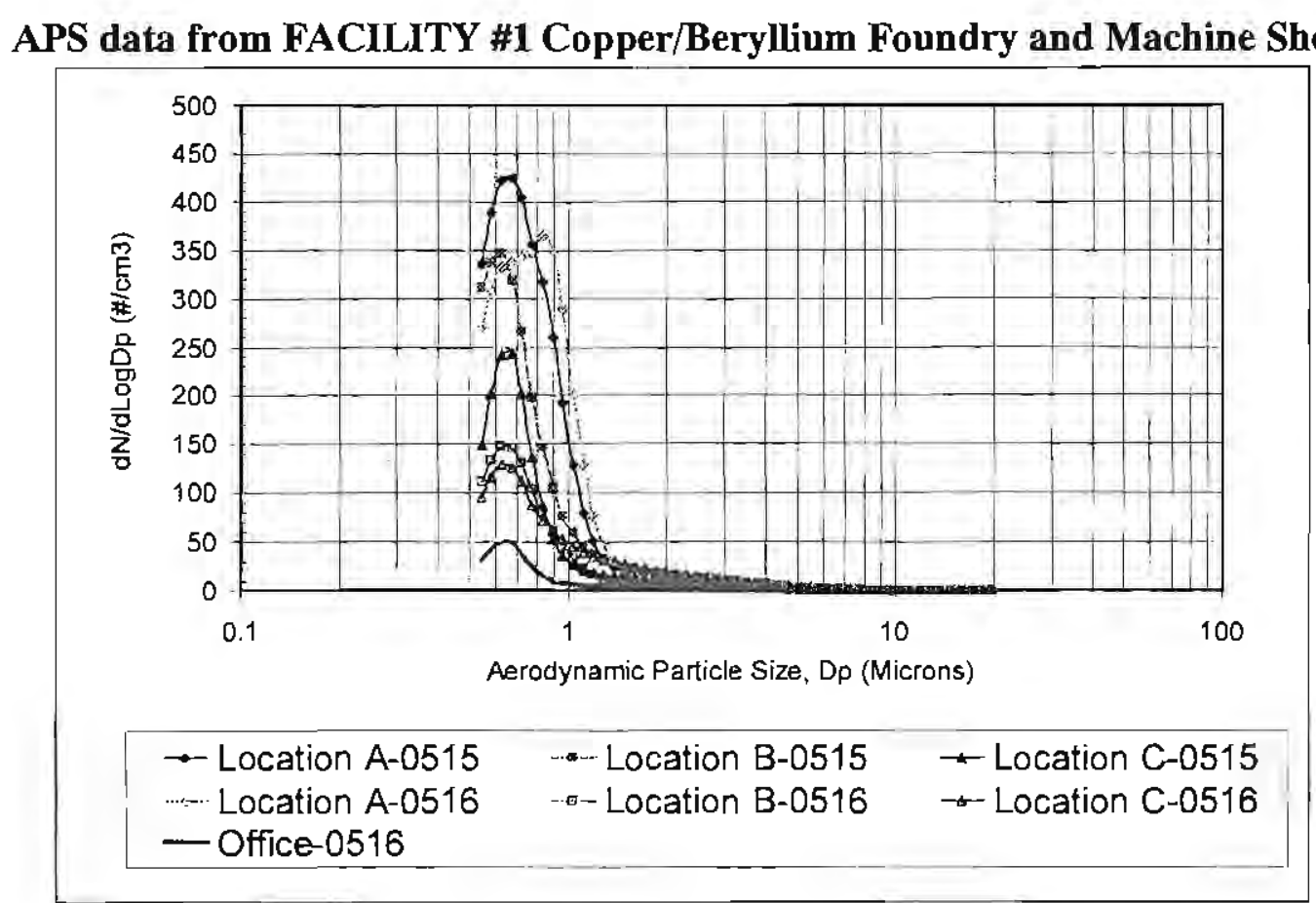


Table 6

BERYLLIUM FACILITY \#1 - Copper/Beryllium Foundry Bulk Dust Sample Results for Beryllium (Be) and Copper $(\mathrm{Cu})$ in $\mathrm{mg} / \mathrm{Kg}=$ milligrams compound per kilogram

\begin{tabular}{|c|l|c|c|c|}
\hline $\begin{array}{c}\text { Sample } \\
\text { Number }\end{array}$ & \multicolumn{1}{|c|}{ Sample Description } & $\begin{array}{c}\text { Sample } \\
\text { Date }\end{array}$ & $\begin{array}{c}\text { Be } \\
(\mathbf{m g} / \mathbf{K g})\end{array}$ & $\begin{array}{c}\text { Cu } \\
(\mathbf{m g} / \mathbf{K g})\end{array}$ \\
\hline SMWB-1 & Settled dust on floor in storage room/Plant 1 & $5 / 16 / 2007$ & 940 & 54000 \\
\hline SMWB-2 & Settled dust on floor near cut-off saw/Plant 1 & $5 / 16 / 2007$ & 2000 & 240000 \\
\hline SMWB-3 & Settled dust on floor core machine room near furnace room wall/Plant 1 & $5 / 16 / 2007$ & 2500 & 220000 \\
\hline SMWB-4 & Settled dust on floor in furnace room behind furnaces/Plant 1 & $5 / 16 / 2007$ & 13000 & 350000 \\
\hline SMWB-5 & Settled dust on floor near cut-off saw/Plant 2 & $5 / 16 / 2007$ & 100 & 11000 \\
\hline
\end{tabular}




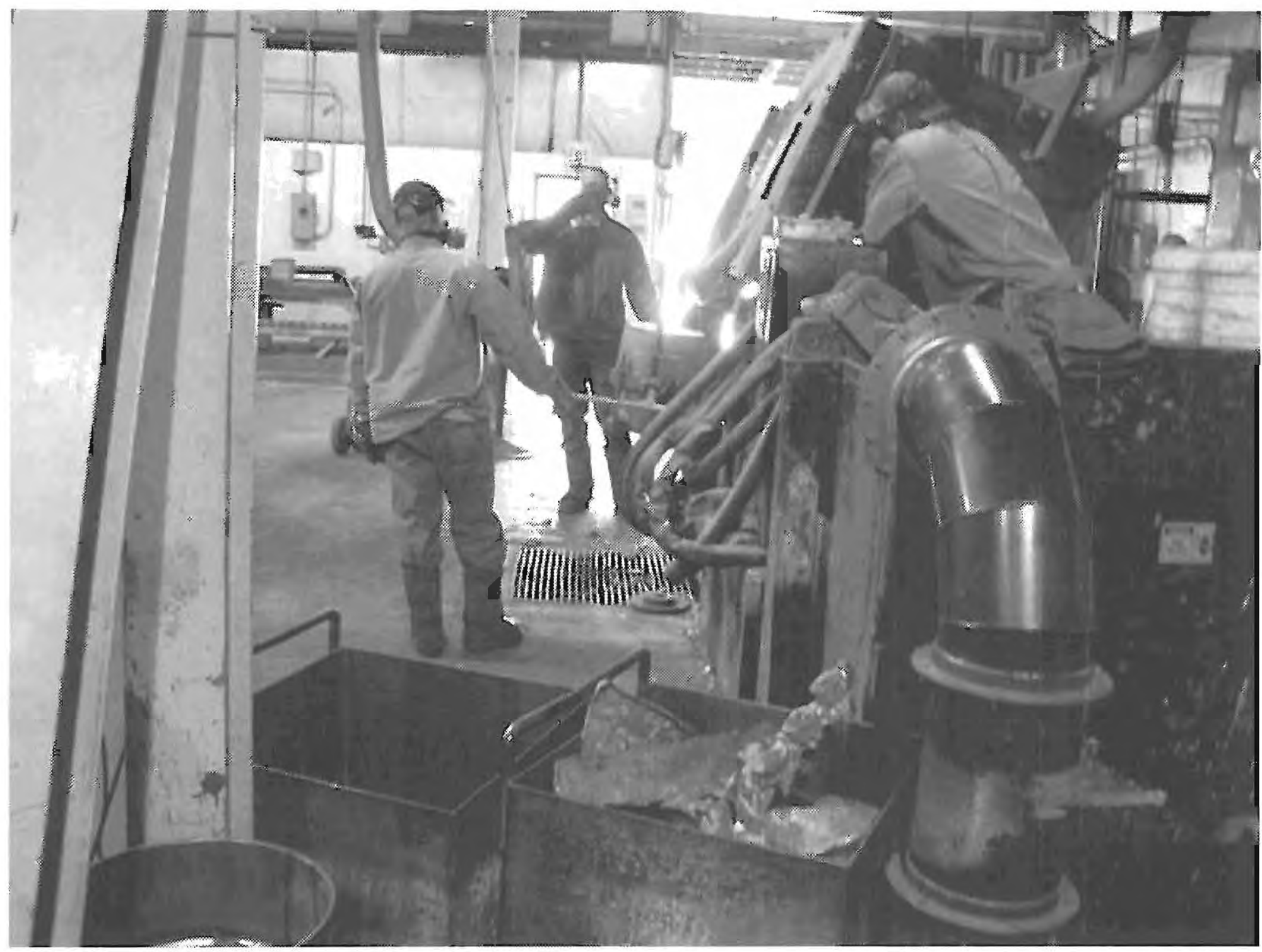

Photo 1 - Plant 2 fumace: The two pourers (standing on the floor) position the crucible while the furnace operator (standing on fumace platform) uses mechanical controls to tilt fumace for pouring molten metal into crucible. The two pourers then transport the crucible to the mold pouring area where they pour the molten metal into molds. The fumace is equipped with slotted hood LEV which is exhausted from the bottom. Flexible ducting is visible in the upper right hand comer of this photo and the slotted hood is visible at the top of the tilted fumace. 


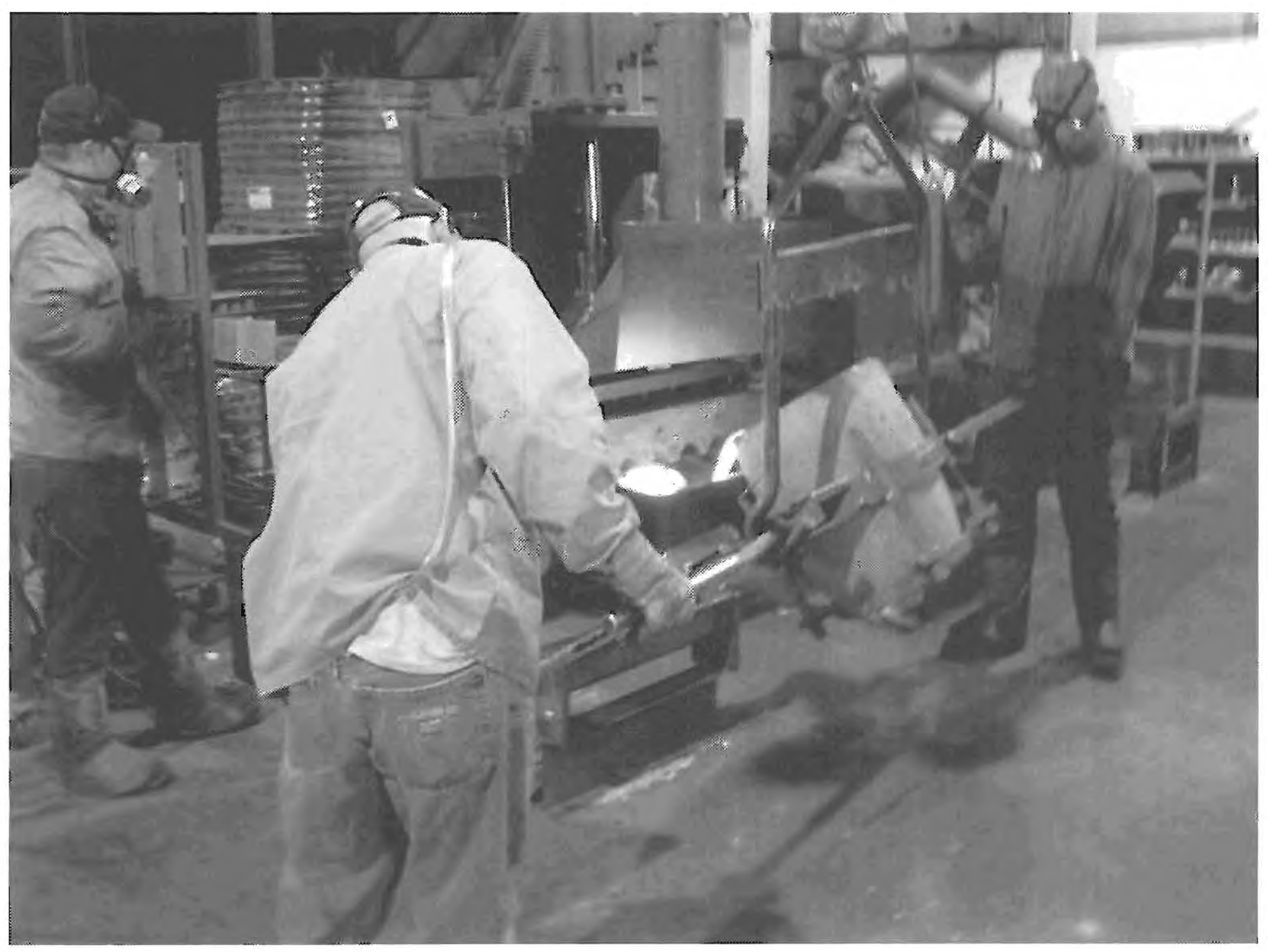

Photo 2 - Plant 2, mold pouring station equipped with local exhaust ventilation hood. The two pourers position crucible to pour molten metal into molds. One pourer operates the crane controls (right) while the second pourer tilts crucible to pour molten metal. The mold pouring process is essentially the same in Plant 1 and Plant 2 of this facility. The major differences are: the crucible transfer mechanism in Plant 1 is equipped with an LEV hood which is positioned over the crucible; and the molds in Plant 1 are placed on a roller conveyor positioned below a slot LEV system attached to the wall (see Photo \#4). 


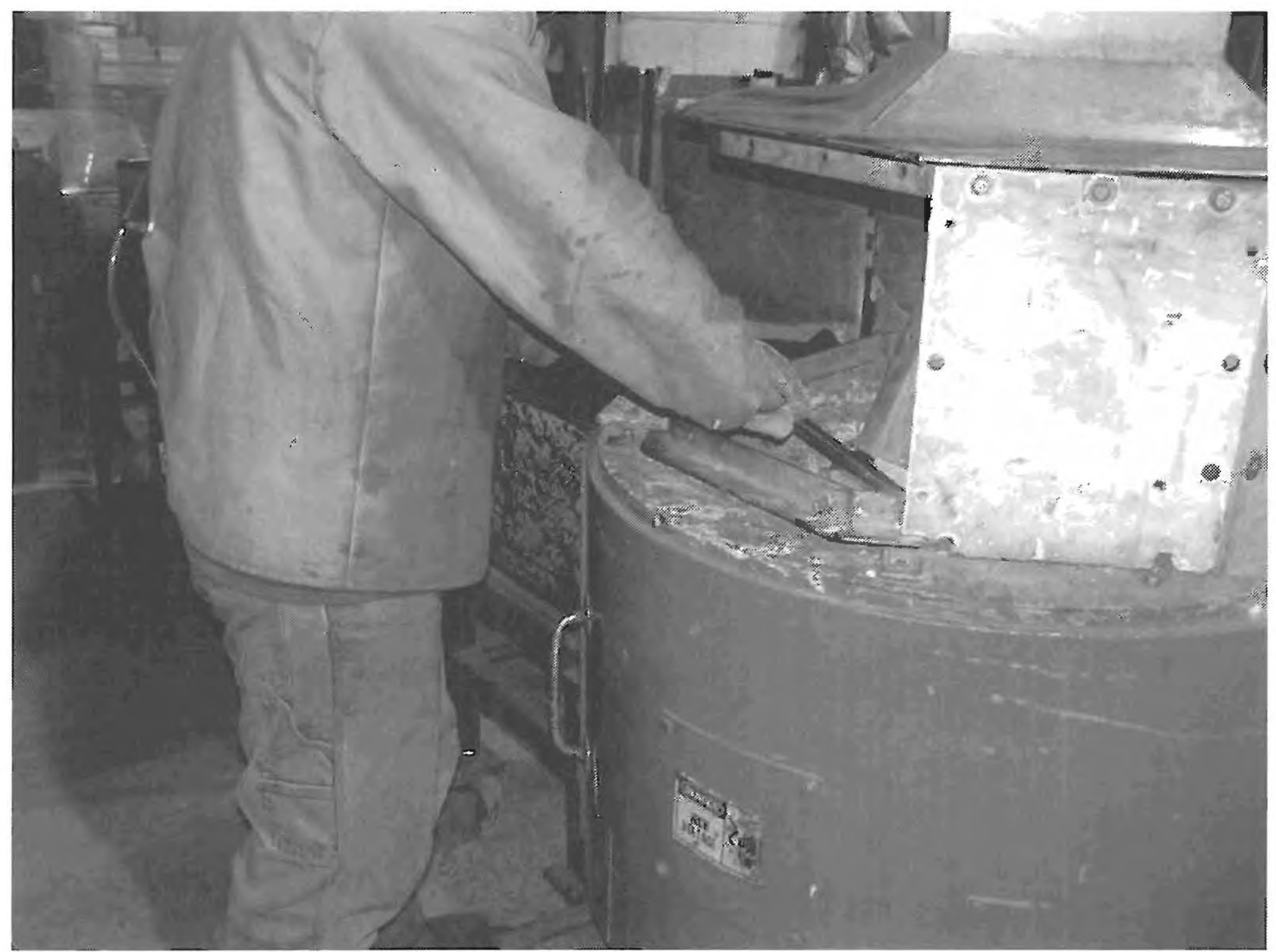

Photo 3 - Plant 1 furnace/ partially enclosing LEV hood. Furnace operator is removing slag from the molten metal. The hood on this furnace is removable to allow the crucible to be removed and transported to the mold pouring stations. 


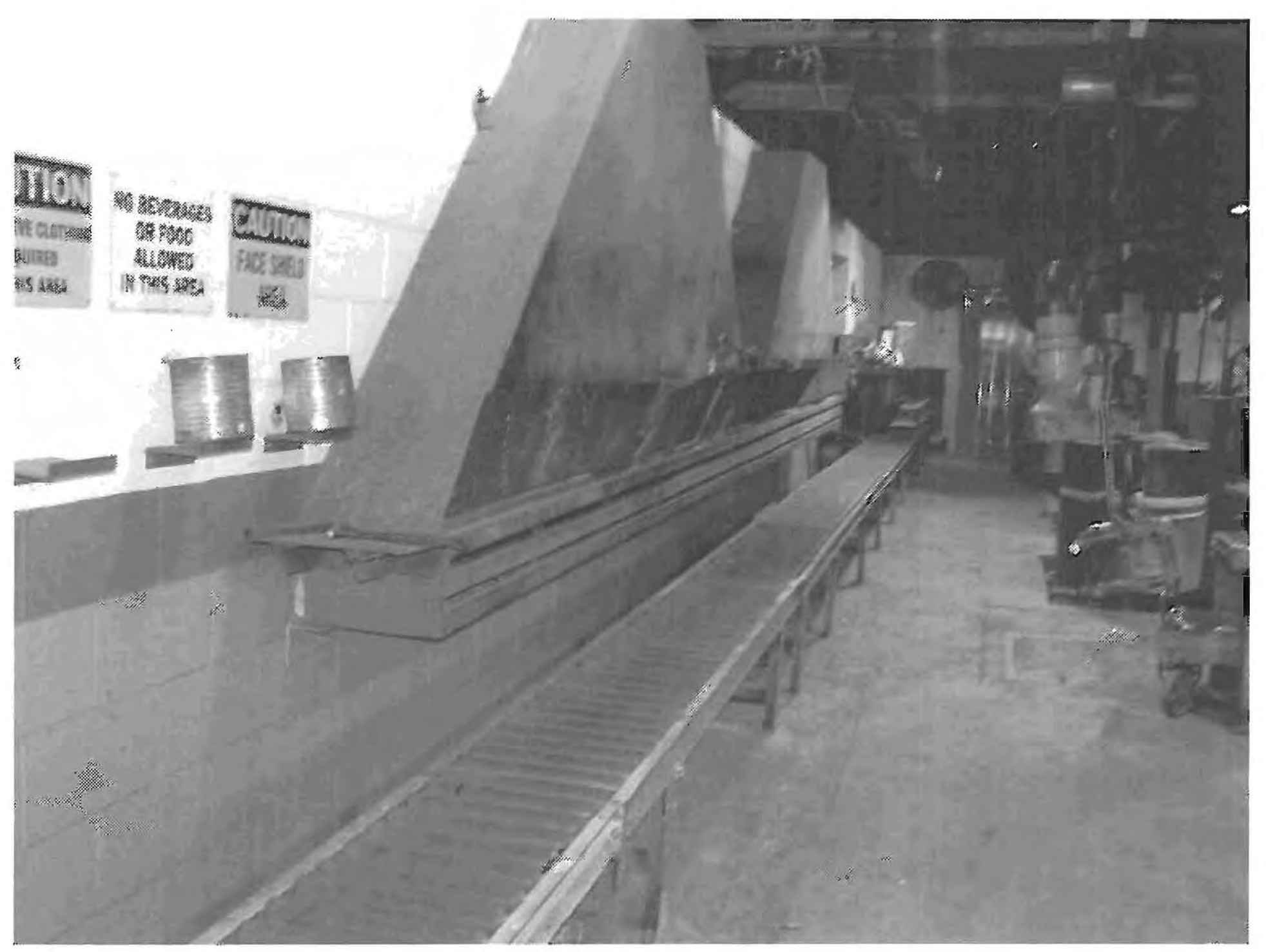

Photo 4 - Plant 1, slot ventilation at the pour rack. Molds in Plant 1 are placed on the roller conveyor for pouring of molten metal into the molds and subsequent transfer to the shake out area. 


\section{Appendix A}

FACILITY \#1 - Copper/Beryllium Foundry and Machine Shop

Personal Breathing Zone and General Area Air sample Result for Thirty-one Elements

\begin{tabular}{|c|c|c|c|c|c|c|c|c|c|c|c|c|c|c|c|c|}
\hline $\begin{array}{l}\text { Sample } \\
\text { Number }\end{array}$ & $\begin{array}{c}\text { A } \\
\text { Conc. } \\
\text { (ug/m3) }\end{array}$ & $\begin{array}{c}\text { sb } \\
\text { Conc. } \\
(\mu g / m 3)\end{array}$ & $\begin{array}{c}\mathrm{Ag} \\
\text { Conc. } \\
(\mu g / m 3)\end{array}$ & $\begin{array}{c}\text { Ba } \\
\text { Conc } \\
(\mu \mathrm{g} / \mathrm{m} 3)\end{array}$ & 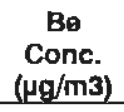 & $\begin{array}{c}c d \\
\text { Conc. } \\
(\mu g / m 3)\end{array}$ & $\begin{array}{c}\mathrm{Ca} \\
\text { Conc. } \\
(\mu g / \mathrm{m} 3)\end{array}$ & $\begin{array}{c}\mathrm{Cr}_{\mathrm{r}} \\
\text { Conc. } \\
(\mu g / \mathrm{m} 3)\end{array}$ & $\begin{array}{c}\text { co } \\
\text { Conc. } \\
(\mu g / m 3)\end{array}$ & $\begin{array}{c}\mathrm{Cu} \\
\text { Conc. } \\
(\mu \mathrm{g} / \mathrm{m} 3)\end{array}$ & $\begin{array}{c}F e \\
\text { Conc. } \\
(\mu \mathrm{g} / \mathrm{m} 3)\end{array}$ & $\begin{array}{c}\text { L } \\
\text { Conc. } \\
(\mu g / m 3)\end{array}$ & $\begin{array}{c}\mathrm{Pb} \\
\text { Conc. } \\
(\mu g / m 3)\end{array}$ & $\begin{array}{l}\text { Li Conc. } \\
(\mu \mathrm{g} / \mathrm{m} 3)\end{array}$ & $\begin{array}{c}\mathrm{Mg} \\
\mathrm{Cona} \\
(\mu \mathrm{g} / \mathrm{m} 3)\end{array}$ & $\begin{array}{c}M n \\
\text { Conc. } \\
(\mu \mathrm{g} / \mathrm{m} 3)\end{array}$ \\
\hline SMTF-7 & 31.8 & $<0.35$ & $<0.35$ & 0.12 & 2.54 & $<0.01$ & 54.3 & 0.39 & 1.91 & 168 & 27.7 & $<0.01$ & 0.42 & 0.027 & 26.0 & 0.49 \\
\hline SMTF-8 & 10.5 & $<0.35$ & $<0.35$ & 0.06 & 0.24 & $<0.01$ & 27.5 & 0.09 & 0.23 & 5.44 & 7.61 & $<0.01$ & $<0.18$ & 0.011 & 17.0 & 0.24 \\
\hline SMTF-9 & 5.09 & $<0.35$ & $<0.35$ & 0.04 & 0.57 & $<0.01$ & 35.7 & 0.17 & 0.56 & 9.36 & 14.0 & $<0.01$ & $<0.18$ & 0.005 & 11.1 & 0.19 \\
\hline SMTF-10 & 7.26 & $<0.36$ & $<0.36$ & 0.03 & 4.72 & 0.029 & 17.5 & 0.60 & 1.45 & 36.9 & 7.26 & 0.04 & $<0.18$ & 0.009 & 11.5 & 0.15 \\
\hline SMTF-11 & 16.2 & $<0.36$ & $<0.36$ & 0.08 & 5.52 & $<0.01$ & 37.8 & 0.28 & 2.58 & 50.4 & 15.6 & $<0.01$ & $<0.18$ & 0.015 & 17.4 & 0.28 \\
\hline SMTF. 12 & 30.2 & $<0.33$ & $<0.33$ & 0.05 & 1.59 & $<0.01$ & 23.6 & 0.21 & 0.48 & 23.1 & 13.2 & $<0.01$ & 0.27 & 0.010 & 14.8 & 0.21 \\
\hline SMTF-13 & 13.6 & $<0.68$ & $<0.68$ & 0.04 & 0.81 & $<0.02$ & 30.6 & 0.52 & 0.69 & 52.1 & 19.2 & $<0.02$ & $<0.34$ & $<0.005$ & 10.3 & 0.29 \\
\hline SMTF-14 & 42.7 & $<0.34$ & $<0.34$ & 0.15 & 1.14 & $<0.01$ & 53.0 & 0.19 & 18.80 & 55.8 & 28.5 & $<0.01$ & 1.48 & 0.037 & 17,1 & 2,11 \\
\hline SMTF-15 & 6.33 & $<0.35$ & $<0.35$ & 0.04 & 0.81 & $<0.01$ & 38.0 & 0.10 & 0.75 & 13.2 & 12.7 & $<0.01$ & $<0.17$ & 0.007 & 14.4 & 0.18 \\
\hline SMTF-16 & $<0.84$ & $<0.50$ & $<0.50$ & $<0.02$ & 0.07 & $<0.02$ & 12.6 & $<0.06$ & $<0.03$ & 3.53 & 2.27 & $<0,02$ & $<0.25$ & $<0.005$ & 3.95 & 0.04 \\
\hline SMTF-17 & $<0.85$ & $<0.51$ & $\leq 0.51$ & $<0.02$ & 0.08 & $<0.02$ & 13.6 & $<0.06$ & 0.05 & 4.34 & 2.21 & $\leq 0.02$ & $<0.26$ & $<0.005$ & 3.49 & 0.03 \\
\hline SMTF-18 & 38.6 & $<0.44$ & $<0.44$ & 0.07 & 1.60 & $<0.01$ & 39.3 & 0.33 & 0.57 & 25.5 & 17.5 & $<0.01$ & $<0.22$ & 0.013 & 18.2 & 0.27 \\
\hline SMTF-19 & 26.5 & $<0.41$ & $<0.41$ & 0.11 & 2.17 & 0.024 & 40.8 & 1.02 & 1.15 & 44.2 & 21.1 & $<0.01$ & 0.28 & 0.025 & 23.1 & 0.31 \\
\hline SMTF-20 & $<0.87$ & $<0.52$ & $<0.52$ & $<0.02$ & 0.06 & $<0.02$ & 10.4 & $<0.06$ & $<0.03$ & 3.72 & 1.39 & $<0.02$ & $<0.26$ & $<0.005$ & 2.25 & 0.02 \\
\hline SMWF-10 & 6.37 & $<0.38$ & $<0.38$ & 0.03 & 2.29 & 0.045 & 17.8 & 0.06 & 1.15 & 36.3 & 8.28 & $<0.01$ & 0.23 & $<0.005$ & 5.93 & 0.15 \\
\hline SMWF-11 & 4.54 & $<0.37$ & $<0.37$ & 0.10 & 0.25 & $<0.01$ & 18.4 & $<0.11$ & 0.29 & 4.66 & 6.75 & $<.01$ & $<0.18$ & $<0.007$ & 9.81 & 0.23 \\
\hline SMWF-12 & 8.21 & $<0.38$ & $<0.38$ & 0.06 & 0.33 & $<0.01$ & 31.0 & $<0.06$ & 0.45 & 6.95 & 9.48 & $<0.01$ & $<0.19$ & 0.010 & 15.8 & 0.25 \\
\hline SMWF-13 & 3.62 & $<0.35$ & $<0.35$ & 0.07 & 0.43 & $<0.01$ & 24.5 & 0.11 & 0.48 & 8.17 & 29.8 & 0.02 & $<0.18$ & $<0.006$ & 8.2 & 0.27 \\
\hline$S M W F=14$ & 17.7 & $<0.35$ & $<0.35$ & 0.08 & 1.30 & $<0.01$ & 40.1 & 0.12 & 0.34 & 31.3 & 14.2 & $<0.01$ & $<0.18$ & 0.018 & 14.8 & 0.35 \\
\hline SMWF-15 & 7.59 & $<0.35$ & $<0.35$ & 0.07 & 4.79 & $<0.01$ & 16.3 & 0.14 & 0.64 & 146 & 15.2 & $<0.01$ & 0.40 & $<0.007$ & 6.42 & 0.15 \\
\hline SMWF-16 & 3.48 & $<0.35$ & $<0.35$ & 0.02 & 0,46 & $<0.01$ & 16.3 & $<0.07$ & 0.64 & 17.4 & 11.0 & $<0.01$ & $<0.17$ & $<0.005$ & 5.52 & 0.13 \\
\hline SMWF-17 & 11.6 & $<0.37$ & $<0.37$ & 0.04 & 1.16 & 0.038 & 12.2 & 0.40 & 0.44 & 42.8 & 9.79 & $<0.01$ & 0.24 & 0.015 & 20.2 & 0.16 \\
\hline SMWF-18 & 17.7 & $<0.44$ & $<0.44$ & 0.03 & 1.40 & 0.017 & 15.5 & 0.37 & 0.52 & 43.6 & 10.3 & $<0.01$ & 0.39 & 0.010 & 13.3 & 0.13 \\
\hline SMWF-19 & 55.9 & $<0.40$ & $<0.40$ & 0.07 & 2.04 & 0.029 & 21.7 & 0.30 & 0.62 & 34.9 & 40.8 & 0.02 & 0.21 & 0.013 & 15.1 & 0.79 \\
\hline SMWF-21 & $<0.79$ & $<0.47$ & $<0.47$ & $<0.02$ & 0.13 & $<0.02$ & 7.56 & $<0.06$ & 0.09 & 6.14 & 1.81 & $<0.02$ & $<0.24$ & $<0.005$ & 1.81 & $<0.02$ \\
\hline SMWF-22 & $<1,11$ & $<0.67$ & $<0.67$ & $<0.02$ & 0.27 & $<0.02$ & 13.3 & $<0.06$ & 0.19 & 11.1 & 3.44 & $<0.02$ & $<0.39$ & $<0.005$ & 3.33 & 0.05 \\
\hline SMWF-23 & $<0.77$ & $<0.46$ & $<0.46$ & $<0.02$ & 0.15 & $<0.02$ & 9.29 & $<0.06$ & 0.08 & 6.04 & 2.63 & $<0.02$ & $\leq 0.23$ & $<0.005$ & 2.48 & 0.02 \\
\hline
\end{tabular}




\section{Appendix A - continued}

FACILITY \#1 - Copper/Beryllium Foundry and Machine Shop

Personal Breathing Zone and General Area Air sample Result for Thirty-one Elements

\begin{tabular}{|c|c|c|c|c|c|c|c|c|c|c|c|c|c|c|c|}
\hline $\begin{array}{l}\text { Sample } \\
\text { Number }\end{array}$ & $\begin{array}{c}\text { Mo } \\
\text { Conc. } \\
(\mu g / m 3)\end{array}$ & $\begin{array}{c}\text { NI } \\
\text { Conc. } \\
(\mu g / m 3)\end{array}$ & $\begin{array}{c}P \\
\text { Conc } \\
(\mu g / m 3)\end{array}$ & $\begin{array}{c}K \\
\text { Conc. } \\
(\mu \mathrm{g} / \mathrm{m} 3)\end{array}$ & $\begin{array}{c}\text { So } \\
\text { Conc. } \\
(\mu g / m 3)\end{array}$ & $\begin{array}{c}\mathrm{Ag} \\
\mathrm{Conc} . \\
(\mu \mathrm{g} / \mathrm{m} 3)\end{array}$ & $\begin{array}{c}\mathrm{Sr} \\
\text { Canc. } \\
(\mu g / m 3)\end{array}$ & $\begin{array}{c}\text { Te } \\
\text { Conc. } \\
(\mu \mathrm{g} / \mathrm{m} 3)\end{array}$ & $\begin{array}{c}\mathrm{Tl} \\
\text { Conc. } \\
(\mu \mathrm{g} / \mathrm{m} 3)\end{array}$ & $\begin{array}{c}\text { Sn } \\
\text { Conc. } \\
(\mu g / m 3)\end{array}$ & $\begin{array}{c}\text { TI } \\
\text { Conc. } \\
(\mu \mathrm{g} / \mathrm{m} 3)\end{array}$ & $\begin{array}{c}v \\
\text { Conc. } \\
(\mu g / m 3)\end{array}$ & $\begin{array}{c}Y \\
\text { Conc. } \\
(\mu g / m 3)\end{array}$ & $\begin{array}{c}\mathrm{Zn} \\
\text { Conc. } \\
(\mu \mathrm{g} / \mathrm{m} 3)\end{array}$ & $\begin{array}{c}\mathrm{Zr} \\
\text { Conc. } \\
(\mu g / \mathrm{m} 3)\end{array}$ \\
\hline SMTF-7 & $<0,06$ & 1.45 & $<0.58$ & 2.6 & $<1.73$ & 0.047 & 0.139 & $<0.23$ & $<0.40$ & $<0.29$ & 0.58 & $<0.012$ & $<0.002$ & 1.39 & $<0.23$ \\
\hline SMTF-B & $<0.06$ & $<0.06$ & $<0.58$ & 1.35 & $<1.76$ & $<0.006$ & 0.076 & $<0.23$ & $<0.41$ & $<0.29$ & 0.44 & $<0.012$ & $<0.002$ & $<0.12$ & $<0.23$ \\
\hline SMTF- 9 & $<0.06$ & 0.15 & $<0.59$ & 1.35 & $\leq 1.76$ & $<0.006$ & 0.059 & $<0.23$ & $<0.41$ & $<0.29$ & 0.12 & $<0.012$ & $<0.002$ & 0.48 & $<0.23$ \\
\hline SMTF-10 & $<0.06$ & 0.50 & $<0.60$ & 1.39 & $<1.81$ & 0.025 & 0.053 & $<0.24$ & $<0.42$ & $<0.30$ & 0.18 & 0.013 & 0.011 & 0.79 & $<0.24$ \\
\hline SMTF-11 & $<0.06$ & 0.72 & $<0.60$ & 1.86 & $<1.80$ & 0.009 & 0.096 & $<0.24$ & $<0.42$ & $<0.30$ & 0.26 & $<0.012$ & $<0.002$ & 0.78 & $<0.24$ \\
\hline SMTF-12 & $<0.05$ & $\underline{0.26}$ & $<0.55$ & 1.32 & $<1.64$ & $<0.005$ & 0.052 & $<0.21$ & $<0.38$ & $<0.27$ & 0.23 & $<0.011$ & $<0.002$ & 0.82 & $<0.22$ \\
\hline SMTF-13 & $<0.11$ & 0.45 & $<1 . \dagger 3$ & 1.47 & $<3,40$ & $<0.011$ & 0.082 & $\leq 0.45$ & $<0.79$ & $<0.57$ & 3.51 & $<0.023$ & $<0.003$ & $<0.23$ & $<0.45$ \\
\hline SMTF-14 & $<0.06$ & 0.68 & $<0.57$ & 2.62 & $<1.71$ & $<0.006$ & 0.154 & $<0.23$ & $<0.40$ & C0.28 & 0.85 & 0.023 & 0.002 & 1.94 & $<0.23$ \\
\hline SMTF-15 & $<0.06$ & 0.11 & $<0.58$ & 1.67 & $<1.73$ & $<0.006$ & 0.069 & $<0.23$ & $<0.40$ & $<0.29$ & 0.14 & $<0.012$ & $<0.002$ & $<0.12$ & $<0.23$ \\
\hline SMTF-16 & $<0.08$ & $<0.08$ & $<0.84$ & $\leq 0.50$ & $<2.52$ & $<0.008$ & 0.009 & $<0.34$ & $<0.59$ & $<0.42$ & 0.03 & $<0.017$ & $<0.003$ & $<0.17$ & $<0.34$ \\
\hline SMTF-17 & $<0.08$ & $<0.09$ & $<0.85$ & $<0.51$ & $<2.55$ & $<0.009$ & 0.009 & $<0.34$ & $<0.60$ & $<0.43$ & 0.03 & $<0.017$ & $<0.003$ & $<0.17$ & $<0.34$ \\
\hline SMTF-18 & $<0.07$ & 0.41 & $<0.73$ & 2.26 & $<2.15$ & $<0.007$ & 0.080 & $<0.29$ & $<0.51$ & $<0.36$ & 0.29 & $<0.015$ & $<0.002$ & 1.02 & $<0.29$ \\
\hline SMTF-19 & $<0.07$ & 0.82 & $<0.68$ & 2.65 & $<2.04$ & 0.027 & 0.129 & $<0.27$ & $<0.48$ & $<0.34$ & 0.30 & $<0.014$ & 0.011 & 1.29 & $<0.27$ \\
\hline SMTF-20 & $<0.09$ & $<0.09$ & $<0.87$ & $<0.52$ & $<2.60$ & $<0.009$ & $<0.009$ & $<0.35$ & $<0.61$ & $<0.43$ & $<0.02$ & $<0.017$ & $<0.003$ & $<0.17$ & $<0.35$ \\
\hline SMWF-10 & $<0.06$ & 0.83 & $<0.64$ & 1.59 & $<1.91$ & 0.009 & 0.030 & $<0.25$ & $<0.45$ & $<0.32$ & 0.13 & $<0.013$ & $<0.002$ & 0.76 & $<0.25$ \\
\hline SMWF-11 & $<0.06$ & $<0.06$ & $<0.61$ & 0.80 & $<1.84$ & $<0.006$ & 0.042 & $<0.25$ & $<0.43$ & $<0.31$ & 0.13 & $<0.012$ & $<0.002$ & $<0.13$ & $<0.25$ \\
\hline SMWF-12 & $<0.06$ & $\leq 0.06$ & $<0.63$ & 1.64 & $<1.00$ & $<0.006$ & 0.076 & $<0.25$ & $<0.44$ & $<0.32$ & 0.32 & $<0.013$ & $<0.002$ & $<0.13$ & $<0.25$ \\
\hline SMWF-13 & $<0.06$ & 0.11 & $<0.58$ & 1.17 & $<1.75$ & $<0.006$ & 0.045 & $<0.23$ & $<0.41$ & $<0.29$ & 0.11 & $<0.012$ & $<0.002$ & 0.50 & $<0.23$ \\
\hline SMWF-14 & $<0.06$ & 0.18 & $<0.59$ & 1.95 & $<1.77$ & $<0.006$ & 0.089 & $<0.24$ & $<0.41$ & $<0.30$ & 0.45 & $<0.012$ & $<0.002$ & 0.29 & $<0.24$ \\
\hline SMWF-15 & $<0.06$ & 0.32 & $<0.58$ & 1.23 & $<1.75$ & 0.035 & 0.050 & $<0.23$ & $<0.41$ & $<0.29$ & 0.15 & $<0.012$ & $<0.002$ & 0.76 & $<0.23$ \\
\hline SMWF -16 & $<0.06$ & 0.10 & $<0.58$ & 0.75 & $<1.74$ & $<0.006$ & 0.024 & $<0.23$ & $<0.41$ & $<0.29$ & 0.08 & $<0.012$ & $<0.002$ & $<0,12$ & $<0.23$ \\
\hline SMWF-17 & $<0.06$ & 0.86 & $<0.61$ & 0.92 & $<1.84$ & 0.029 & 0.043 & $<0.24$ & $<0.43$ & $<0.31$ & 0.13 & 0.016 & 0.009 & 1.04 & $<0.24$ \\
\hline SMWF-18 & $<0.07$ & 0.81 & $<0.74$ & 1.03 & $<2.21$ & 0.010 & 0.050 & $<0.30$ & $<0.52$ & $<0.37$ & 0.15 & $<0,015$ & $<0.002$ & 0.81 & $<0.30$ \\
\hline SMWF-19 & $<0.07$ & 0.47 & $<0.66$ & 1.71 & $<1.97$ & 0.009 & 0.059 & $<0.26$ & $<0.46$ & $<0.33$ & 0.25 & $<0.013$ & $<0.002$ & 1.38 & $<0.26$ \\
\hline SMWF-21 & $<0.08$ & $<0.08$ & $<0.79$ & $<0.47$ & $<2.36$ & $<0.008$ & 0.013 & $<0.31$ & $<0.55$ & $<0.39$ & 0.02 & $<0.016$ & $<0.002$ & $<0.16$ & $<0.31$ \\
\hline SMWF-22 & $<0.11$ & $<0.11$ & $<1.11$ & $\leq 0.67$ & $<3.33$ & $<0.011$ & 0.024 & $<0.44$ & $<0.78$ & $<0.55$ & 0.04 & $<0.022$ & $<0.003$ & $<0.22$ & $<0.44$ \\
\hline SMWF-23 & $<0.08$ & $<0.08$ & $<0.77$ & 0.66 & $<2.32$ & $<0.008$ & 0.015 & $<0.31$ & $<0.54$ & $<0.39$ & 0.03 & $<0.015$ & $<0.002$ & $<0.15$ & $<0.31$ \\
\hline
\end{tabular}




\section{Appendix B}

FACILITY \#1 - Copper/Beryllium Foundry and Machine Shop Surface Wipe Sample Results for Thirty-one Elements

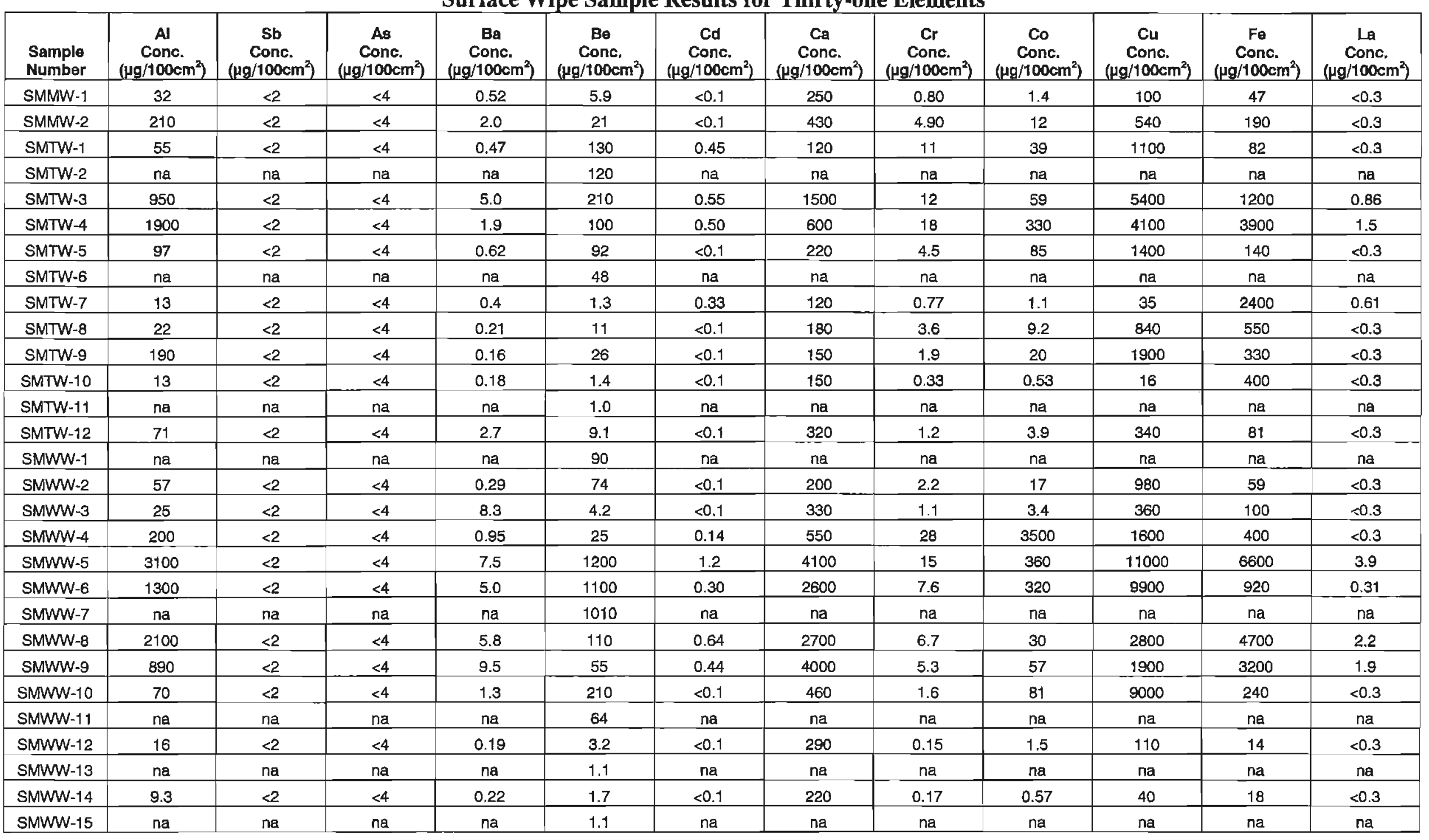




\section{Appendix B - continued}

FACILITY \#1 - Copper/Beryllium Foundry and Machine Shop Surface Wipe Sample Results for Thirty-one Elements

\begin{tabular}{|c|c|c|c|c|c|c|c|c|c|c|c|}
\hline $\begin{array}{l}\text { Semple } \\
\text { Number }\end{array}$ & $\begin{array}{c}\mathrm{Pb} \\
\text { Conc. } \\
\left(\mu g / 100 \mathrm{em}^{2}\right)\end{array}$ & $\begin{array}{c}\mathrm{LI} \\
\text { Conc. } \\
\left(\mu \mathrm{g} / 100 \mathrm{~cm}^{2}\right)\end{array}$ & $\begin{array}{c}\mathrm{Mg} \\
\text { Conc. } \\
\left(\mu \mathrm{g} / 100 \mathrm{~cm}^{2}\right)\end{array}$ & $\begin{array}{c}M n \\
\text { Conc. } \\
\left(\mu g / 100 \mathrm{~cm}^{2}\right)\end{array}$ & $\begin{array}{c}\text { Mo } \\
\text { Conc. } \\
\left(\mu g / 100 \mathrm{em}^{2}\right)\end{array}$ & $\begin{array}{c}N i \\
\text { Conc. } \\
\left(\mu g / 100 \mathrm{~cm}^{2}\right)\end{array}$ & $\begin{array}{c}P \\
\text { Conc. } \\
\left(\mu \mathrm{g} / 100 \mathrm{~cm}^{2}\right)\end{array}$ & $\begin{array}{c}K \\
\text { Conc. } \\
\left(\mu \mathrm{g} / 100 \mathrm{~cm}^{2}\right)\end{array}$ & $\begin{array}{c}\text { Se } \\
\text { Conc. } \\
\left(\mu \mathrm{g} / 100 \mathrm{~cm}^{2}\right)\end{array}$ & $\begin{array}{c}A g \\
\text { Conc. } \\
\left(\mu g / 100 \mathrm{~cm}^{2}\right)\end{array}$ & $\begin{array}{c}\text { Sr } \\
\text { Conc. } \\
\left(\mu \mathrm{g} / 100 \mathrm{~cm}^{2}\right)\end{array}$ \\
\hline SMMW-1 & 0.6 & $<0.09$ & 59 & 0.89 & $<0.2$ & $<3$ & $<30$ & 23 & $<5$ & $<0.03$ & 0.87 \\
\hline SMMW-2 & 1.8 & 0.19 & 310 & 3.3 & 0.21 & 6.1 & $<30$ & 40 & $<5$ & 0.12 & 1.8 \\
\hline SMTW-1 & 3 & 0.14 & 130 & 1.8 & 0.46 & 15 & $<30$ & 59 & $<5$ & 0.28 & 0.48 \\
\hline SMTW-2 & na & na & na & na & ne & na & ne & na & ne & na & na \\
\hline SMTW-3 & 16 & 1.5 & 1900 & 22 & 0.67 & 63 & $<30$ & 220 & $<5$ & 1.5 & 5.8 \\
\hline SMTW-4 & 11 & 0.32 & 210 & 31 & 3.5 & 74 & $<30$ & 64 & $<5$ & 0.93 & 1.6 \\
\hline SMTW-5 & 3.4 & 0.12 & 66 & 3.0 & 0.24 & 24 & $<30$ & 90 & $<5$ & 0.35 & 0.83 \\
\hline SMTW-6 & na & ne & na & $\mathrm{ne}$ & ne & na & na & na & na & na. & na \\
\hline SMTW-7 & 0.44 & $<0.09$ & 11 & 16 & 0.4 & $<3$ & $<30$ & 11 & $<5$ & $<0.03$ & 0.2 \\
\hline SMTW-8 & 2.0 & $<0.09$ & 16 & 6.9 & $<0.2$ & 6.7 & $<30$ & 11 & $<5$ & 0.18 & 0.59 \\
\hline SMTW-9 & 4.1 & $<0.09$ & $<4$ & 6.4 & $<0.2$ & 15 & $<30$ & $<7$ & $<5$ & 0.44 & 0.27 \\
\hline SMTW-10 & $<0.3$ & $<0.09$ & 12 & 2.6 & $<0.2$ & $<3$ & $<30$ & 25 & $<5$ & $<0.03$ & 0.15 \\
\hline SMTW-11 & na & na & na & na & na & na & na & na & na & na & na \\
\hline SMTW-12 & 1.0 & $<0.09$ & 140 & 2.6 & $<0.2$ & 4.1 & $<30$ & 170 & $<5$ & 0.076 & 1.6 \\
\hline SMWW-1 & na & na & na & na & na & na & na & na & na & no & na \\
\hline SMWW-2 & 2.3 & $<0.09$ & 34 & 1.1 & $<0.2$ & 9.3 & $<30$ & 22 & $<5$ & 0.25 & 0.25 \\
\hline SMWW-3 & 1.4 & $<0.09$ & 63 & 1.8 & $<0.2$ & 3.6 & $<30$ & 53 & $<5$ & 0.076 & 1.1 \\
\hline SMWW-4 & 21.0 & 0.16 & 100 & 41 & 0.76 & 37 & $<30$ & 62 & $<5$ & 0.43 & 1.5 \\
\hline SMWW-5 & 25.0 & 2.6 & 1100 & 88 & 1.5 & 220 & $<30$ & 410 & $<5$ & 2.4 & 9.6 \\
\hline SMWW-6 & 21 & 1.4 & 600 & 20 & 0.93 & 150 & $<30$ & 260 & $<5$ & 2.2 & 6.1 \\
\hline SMWW-7 & na & na & na & $\mathrm{na}$ & na & na & na & na & na & na & na \\
\hline SMWW-8 & 8.2 & 2.2 & 820 & 56 & 0.51 & 38 & $<30$ & 300 & $<5$ & 0.66 & 7.6 \\
\hline SMWW-9 & 6.8 & 4.6 & 2500 & 43 & 0.96 & 22 & $<30$ & 350 & $<5$ & 0.41 & 19 \\
\hline SMWW-10 & 16.0 & 0.20 & 45 & 3.1 & 0.20 & 49 & $<30$ & 89 & $<5$ & 1.9 & 0.82 \\
\hline SMWW-11 & na & na & na & na & na & na & na & na & na & ne & na \\
\hline SMWW-12 & 0.60 & $<0.09$ & $<4$ & 0.45 & $<0.2$ & $<3$ & $<30$ & 48 & $<5$ & $<0,03$ & 0.29 \\
\hline SMWW-13 & na & na & na & na & na & na & na & na & na & กа & na \\
\hline SMWW-14 & $<0.3$ & $<0.09$ & 11 & 0.41 & $<0.2$ & $<3$ & $<30$ & 13 & $<5$ & $<0.03$ & 0.40 \\
\hline SMWW-15 & na & na & na & $\mathrm{ne}$ & na & na & na & na & na & na & na \\
\hline
\end{tabular}




\begin{tabular}{|c|c|c|c|c|c|c|c|c|}
\hline \multicolumn{9}{|c|}{$\begin{array}{l}\text { FACILITY \#1 - Copper/Beryllium Foundry and Machine Shop } \\
\text { Surface Wipe Sample Results for Thirty-one Elements }\end{array}$} \\
\hline $\begin{array}{l}\text { Semple } \\
\text { Number }\end{array}$ & $\begin{array}{l}\text { To } \\
\text { Conc. } \\
\left.(\mu g / 100 \mathrm{em})^{2}\right)\end{array}$ & $\begin{array}{c}\text { Conc. } \\
\left(\mu \mathrm{g} / 100 \mathrm{~cm} \mathrm{~m}^{2}\right)\end{array}$ & $\begin{array}{c}\text { Sn } \\
\text { Conc. } \\
\left(\mu g / 100 \mathrm{~cm}^{2}\right)\end{array}$ & $\begin{array}{c}7 \\
\text { Conc. } \\
\left(\mu g / 100 \mathrm{~cm} m^{2}\right)\end{array}$ & $\begin{array}{l}\mathrm{V} \\
\text { Conc. } \\
\left(\mu g / 100 \mathrm{~cm}^{2}\right)\end{array}$ & $\begin{array}{c}\mathrm{Y} \\
\text { Conc. } \\
\left(\mu \mathrm{g} / 100 \mathrm{~cm}^{2}\right)\end{array}$ & $\begin{array}{l}\mathrm{Zn} \\
\text { Conc. } \\
\left(\mu \mathrm{g} / 100 \mathrm{~cm}^{2}\right)\end{array}$ & $\begin{array}{c}\mathrm{Zr} \\
\text { Conc. } \\
\left(\mathrm{\mu g} / 100 \mathrm{~cm}^{2}\right)\end{array}$ \\
\hline SMMW-1 & $<0.2$ & $<9$ & $<2$ & $<2$ & $<0.02$ & 0.013 & 12 & $<20$ \\
\hline SMMW-2 & $<0.2$ & $<9$ & 2.9 & 2.3 & 0.15 & 0.034 & 19 & $<20$ \\
\hline SMTW- 1 & $<0.2$ & $<9$ & $<2$ & 2.2 & 0.097 & 0.026 & 20 & $<20$ \\
\hline SMTW-2 & na & na & na & na & na & na & na & na \\
\hline SMTW-3 & 0.31 & $<9$ & $<2$ & 12 & 0.77 & 0.19 & 79 & $<20$ \\
\hline SMTW-4 & 0.26 & $<9$ & $<2$ & 4.7 & 1.6 & 0.058 & 77 & $<20$ \\
\hline SMTW-5 & $<0.2$ & $<9$ & $<2$ & 2.6 & 0.16 & 0.018 & 27 & $<20$ \\
\hline SMTW-6 & na & na & na & na & nа & na & na & na \\
\hline SMTW-7 & $<0.2$ & $<9$ & $<2$ & $<2$ & $<0.02$ & 0.041 & 8.1 & 20 \\
\hline SMTW-8 & $<0.2$ & $<9$ & $\leq 2$ & $<2$ & $<0.02$ & $<0.01$ & 9.9 & $<0$ \\
\hline SMTW-S & $<0.2$ & $<9$ & $<2$ & $<2$ & $<0.02$ & 0.011 & 16 & $<20$ \\
\hline SMTW-10 & $<0.2$ & $<9$ & 2 & $<2$ & $<0.02$ & $<0.01$ & 5.9 & $<20$ \\
\hline SMTW-11 & na & na & ne & na & na & na & na & กв \\
\hline SMTW-12 & $<0.2$ & $<9$ & 4.7 & $<2$ & 0.049 & 0.013 & 11 & $<20$ \\
\hline SMWW-1 & na & па & na & na & na & na & na & $n a$ \\
\hline SMWW-2 & $<0.2$ & $<9$ & $<2$ & $<2$ & 0.047 & 0.012 & 11 & $<20$ \\
\hline SMWW 3 & $<0.2$ & $<9$ & $<2$ & $<2$ & 0.046 & 0.015 & 11 & $<20$ \\
\hline SMWW-4 & 0.27 & $<9$ & $<2$ & $<2$ & 0.21 & 0.031 & 38 & $<20$ \\
\hline SMWW-5 & 0.73 & $<9$ & $<2$ & 39 & 1.0 & 0.35 & 410 & $<20$ \\
\hline SMWW-6 & 0.56 & $<9$ & $<2$ & 28 & 1.3 & 0.22 & 120 & $<20$ \\
\hline SMWW-7 & na & na & na & na & na & na & na & na \\
\hline SMWW-B & 0.40 & $<9$ & $<2$ & 24 & 0.39 & 0.32 & 61 & $<20$ \\
\hline SMWW-9 & 0.38 & $<9$ & $<2$ & 21 & 0.85 & 0.46 & 53 & $<20$ \\
\hline SMWW-10 & 0.24 & $<9$ & $<2$ & $<2$ & $<0.02$ & 0.034 & 67 & $<20$ \\
\hline SMWW-11 & na & na & na & na & na & ne & na & na \\
\hline SMWW-12 & $<0.2$ & $<9$ & $<2$ & $<2$ & $<0.02$ & $<0.01$ & 9.4 & $<20$ \\
\hline SMWW-13 & na & na & na & па & na & na & na & na \\
\hline SMWN-14 & $<0.2$ & $<9$ & $<2$ & $<$ & $<0.02$ & $<0.01$ & 6.4 & $<20$ \\
\hline SMWN-15 & na & na & na & па & na & na & na & na \\
\hline
\end{tabular}




\begin{tabular}{|c|c|c|c|c|c|c|c|c|c|c|c|c|c|}
\hline \multicolumn{14}{|c|}{$\begin{array}{l}\text { FACILITY \#1 - Copper/Beryllium Foundry and Machine Shop } \\
\text { Sioutas Cascade Impactor, Size-Selective Sample Results for Thirty-one Elements }\end{array}$} \\
\hline $\begin{array}{c}\text { Sample } \\
\text { Number }\end{array}$ & $\begin{array}{l}\text { Particle } \\
\text { Slze } \\
(\mu \mathrm{m})\end{array}$ & $\begin{array}{c}\text { Al } \\
\text { Conc. } \\
\left(\mu g / \mathrm{m}^{3}\right)\end{array}$ & $\begin{array}{c}\text { Sb } \\
\text { Conc. } \\
\left(\mu g / m^{3}\right)\end{array}$ & $\begin{array}{c}\text { AB } \\
\text { Conc. } \\
\left(\mu g / m^{3}\right)\end{array}$ & $\begin{array}{c}\text { Ba } \\
\text { Conc. } \\
\left(\mu g / \mathrm{m}^{3}\right)\end{array}$ & $\begin{array}{c}\mathrm{B \theta} \\
\text { Conc. } \\
\left(\mu \mathrm{g} / \mathrm{m}^{3}\right)\end{array}$ & $\begin{array}{c}\text { cd } \\
\text { conc. } \\
\left(\mu g / \mathrm{m}^{3}\right)\end{array}$ & $\begin{array}{c}\mathrm{Ca}_{\mathrm{a}} \\
\text { Conc. } \\
\left(\mu \mathrm{g} / \mathrm{m}^{3}\right)\end{array}$ & $\begin{array}{c}\mathrm{Cr} \\
\text { Conc. } \\
\left(\mu \mathrm{g} / \mathrm{m}^{3}\right)\end{array}$ & $\begin{array}{c}\text { Co } \\
\text { Conc. } \\
\left(\mu g / \mathrm{m}^{3}\right)\end{array}$ & $\begin{array}{c}\mathrm{cu} \\
\text { Conc. } \\
\left(\mu \mathrm{g} / \mathrm{m}^{3}\right)\end{array}$ & $\begin{array}{c}\mathrm{Fe} \\
\text { Conc. } \\
\left(\mu \mathrm{g} / \mathrm{m}^{3}\right)\end{array}$ & $\begin{array}{c}\text { La } \\
\text { Conc. } \\
\left(\mu \mathrm{g} / \mathrm{m}^{3}\right)\end{array}$ \\
\hline SMTF-1A & 2.5 & 20.1 & $<0.1$ & $<0.2$ & 0.06 & 0.90 & $<0.02$ & 26.1 & 0.34 & 0.52 & 28.1 & 13.3 & $<0.004$ \\
\hline SMTF-1B & 1.0 & 3.2 & $<0.1$ & $<0.2$ & 0.01 & 0.08 & $<0.02$ & 2.2 & $<0.02$ & 0.03 & 2.2 & 1.3 & $<0.004$ \\
\hline SMTF-1C & 0.50 & 0.6 & $<0.1$ & $<0.2$ & $<0.004$ & 0.01 & $<0.02$ & 0.4 & $<0.02$ & $<0.01$ & $<0.2$ & 0.1 & $<0.004$ \\
\hline SMTF-1D & 0.25 & 1.7 & $<0.1$ & $<0.2$ & $<0.004$ & 0.02 & $<0.02$ & $<0.4$ & $<0.02$ & $<0.01$ & $<0.2$ & 0.1 & $<0.004$ \\
\hline SMTF-1E & $<0.25$ & 0.5 & $<0.1$ & $<0.2$ & $<0.004$ & 0.02 & 0.02 & $<0.4$ & $<0.02$ & $<0.01$ & $<0.2$ & $<0.1$ & $<0.004$ \\
\hline SMTF-1 & Total & 26.1 & $<0.1$ & $<0.2$ & 0.07 & 1.03 & 0.02 & 28.8 & 0.1 & 0.56 & 30.3 & 14.8 & $<0.004$ \\
\hline SMTF-2A & 2.5 & 5.3 & $<0.1$ & $<0.2$ & 0.04 & 1.23 & $<0.02$ & 19.1 & 0.13 & 3.29 & 15.6 & 7.6 & $<0.004$ \\
\hline SMTF-2B & 1.0 & 1.9 & $<0.1$ & $<0.2$ & 0.01 & 0.39 & $<0.02$ & 4.9 & 0.04 & 0.68 & 4.3 & 2.0 & $<0.004$ \\
\hline SMTF-2C & 0.50 & 1.8 & $<0.1$ & $<0.2$ & 0.00 & 0.12 & $<0.02$ & 1.2 & $<0.02$ & 0.11 & 0.9 & 0.5 & $<0.004$ \\
\hline SMTF-2D & 0.25 & 0.5 & $<0.1$ & $<0.2$ & $<0.004$ & 0.13 & $<0.02$ & $<0.4$ & $<0.02$ & 0.03 & 0.3 & 0.1 & $<0.004$ \\
\hline SMTF-2E & $<0.25$ & $<0.4$ & $<0.1$ & $<0.2$ & $<0.004$ & 0.14 & 0.03 & $<0.4$ & 0.02 & 0.05 & 1.4 & 0.2 & $<0.004$ \\
\hline SMTF-2 & Total & 9.5 & $<0.1$ & $<0.2$ & 0.06 & 2.01 & 0.03 & 25.3 & 0.18 & 4.16 & 22.6 & 10.4 & $<0.004$ \\
\hline SMTF-3A & 2.5 & 8.0 & $<0.1$ & $<0.2$ & 0.04 & 0.73 & $<0.03$ & 20.0 & 0.16 & 0.67 & 56.7 & 13.4 & $<0.007$ \\
\hline SMTF-3B & 1.0 & $<0.7$ & $<0.1$ & $<0.2$ & $<0.007$ & 0.01 & $<0.03$ & $\leq 0.7$ & $<0.03$ & $<0.01$ & 0.3 & 0.2 & $<0.007$ \\
\hline SMTF-3C & 0.50 & 1.1 & $<0.1$ & $<0.2$ & $<0.007$ & $<0.007$ & $<0.03$ & $<0.7$ & $<0.03$ & $<0.01$ & $<0.3$ & $<0.2$ & $<0.007$ \\
\hline SMTF-3D & 0.25 & 0.9 & $<0.1$ & $<0.2$ & $<0.007$ & $<0.007$ & $<0.03$ & $<0.7$ & $<0.03$ & $<0.01$ & $<0.3$ & $<0.2$ & $<0.007$ \\
\hline SMTF-3E & $<0.25$ & $<0.7$ & $<0.1$ & $<0.2$ & $<0.007$ & $<0.007$ & $<0.03$ & $<0.7$ & $<0.03$ & $<0.01$ & $<0.3$ & $<0.2$ & $<0.007$ \\
\hline SMTF-3 & Total & 10.1 & $<0.1$ & $<0.2$ & 0.04 & 0.74 & $<0.03$ & 20.0 & 0.16 & 0.67 & 57.1 & 13.6 & $<0.007$ \\
\hline SMTF-4A & 2.5 & 6.5 & $<0.1$ & $<0.2$ & 0.04 & 0.61 & $<0.02$ & 12.9 & 0.15 & 0.34 & 24.2 & 5.7 & $<0.004$ \\
\hline SMTF-4B & 1.0 & 2.6 & $<0.1$ & $<0.2$ & 0.01 & 0.20 & $<0.02$ & 3.8 & 0.06 & 0.13 & 8.9 & 1.9 & $<0.004$ \\
\hline SMTF-4C & 0.50 & 1.2 & $<0.1$ & $<0.2$ & 0.01 & 0.07 & $<0.02$ & 1.1 & $<0.02$ & 0.05 & 3.0 & 0.6 & $<0.004$ \\
\hline SMTF-4D & 0.25 & 1.3 & $<0.1$ & $<0.2$ & 0.01 & 0.03 & $<0.02$ & $<0.4$ & $<0.02$ & 0.01 & 0.7 & 0.2 & $<0.004$ \\
\hline SMTF-4E & $<0.25$ & 0.9 & $<0.1$ & $<0.2$ & 0.01 & 0.07 & 0.04 & $<0.4$ & 0.03 & 0.03 & 2.0 & 0.5 & $<0.004$ \\
\hline SMTF-4 & Total & 12.5 & $<0.1$ & $<0.2$ & 0.07 & 0.98 & 0.04 & 17.8 & 0.24 & 0.56 & 38.8 & 8.8 & $<0.004$ \\
\hline
\end{tabular}




\begin{tabular}{|c|c|c|c|c|c|c|c|c|c|c|c|c|c|}
\hline \multicolumn{14}{|c|}{$\begin{array}{l}\text { FACILITY \#1 - Copper/Beryllium Foundry and Machine Shop } \\
\text { Sioutas Cascade Impactor, Size-Selective Sample Results for Thirty-one Elements }\end{array}$} \\
\hline $\begin{array}{l}\text { Sample } \\
\text { Number }\end{array}$ & $\begin{array}{l}\text { Particle } \\
\text { Slze } \\
(\text { Hm) }\end{array}$ & $\begin{array}{c}\text { AJ } \\
\text { Conc. } \\
\left(\mu g / m^{3}\right)\end{array}$ & $\begin{array}{c}\text { Sb } \\
\text { Conc. } \\
\left(\mu g / m^{3}\right)\end{array}$ & $\begin{array}{c}\text { As } \\
\text { Conc. } \\
\left(\mu g / m^{3}\right)\end{array}$ & $\begin{array}{c}\text { Be } \\
\text { Conc. } \\
\left(\mu g / m^{3}\right)\end{array}$ & $\begin{array}{c}\text { Bo } \\
\text { Conc. } \\
\left(\mu \mathrm{g} / \mathrm{m}^{3}\right)\end{array}$ & $\begin{array}{c}\text { Cd } \\
\text { Conc, } \\
\left(\mu g / \mathrm{m}^{3}\right)\end{array}$ & $\begin{array}{c}\mathrm{Ca} \\
\mathrm{Conc} \\
\left(\mathrm{\mu g} / \mathrm{m}^{3}\right)\end{array}$ & $\begin{array}{c}\text { Cr } \\
\text { Conc. } \\
\left(\mu \mathrm{g} / \mathrm{m}^{3}\right)\end{array}$ & $\begin{array}{c}\text { Co } \\
\text { Conc. } \\
\left(\mathrm{g} / \mathrm{m}^{3}\right)\end{array}$ & $\begin{array}{c}\text { cu } \\
\text { Conc. } \\
\left(\mu g / m^{3}\right)\end{array}$ & $\begin{array}{c}\text { Fe } \\
\text { Conc. } \\
\left(\mu g / m^{3}\right)\end{array}$ & $\begin{array}{c}\text { La } \\
\text { Conc. } \\
\left(\mu \mathrm{g} / \mathrm{m}^{2}\right) \\
\end{array}$ \\
\hline SMTF-5A & 2.5 & 2.8 & $<0.1$ & $<0.2$ & 0.03 & 0.22 & $<0.02$ & 16.5 & 0.02 & 0.33 & 4.9 & 6.3 & $<0.004$ \\
\hline SMTF-5B & 1.0 & 0.6 & $<0.1$ & $<0.2$ & 0.01 & 0,04 & $<0.02$ & 3.1 & $<0.02$ & 0.06 & 0.8 & 1.3 & $<0.004$ \\
\hline SMTF-SC & 0.50 & $<0.4$ & $<0.1$ & $<0.2$ & $<0.004$ & 0.01 & $<0.02$ & 0.5 & $<0.02$ & $<0.01$ & $<0.2$ & 0.2 & $<0.004$ \\
\hline SMTF-5D & 0.25 & 0.6 & $<0.1$ & $<0.2$ & $<0.004$ & $<0.004$ & $<0.02$ & $\infty 0.4$ & $<0.02$ & $<0.01$ & $<0.2$ & 0.1 & $<0.004$ \\
\hline SMTF-5E & $<0.25$ & $<0.4$ & $<0.1$ & $<0.2$ & $<0.004$ & $<0.004$ & $<0.02$ & $<0.4$ & $<0.02$ & $<0.01$ & $<0.2$ & $<0.1$ & $<0.004$ \\
\hline SMTF-5 & Total & 3.8 & $<0.1$ & $<0.2$ & 0.04 & 0.27 & $<0.02$ & 20.2 & 0.02 & 0.39 & 5.7 & 7.9 & $<0.004$ \\
\hline SMTF-6A & 2,5 & 4.0 & $<0.1$ & $\leq 0.2$ & 0.04 & 0.16 & $<0.02$ & 19.9 & $<0.02$ & 0.21 & 4,6 & 5.6 & $<0.004$ \\
\hline SMTF-6B & 1.0 & 1.6 & $<0.1$ & $<0.2$ & 0.01 & 0.05 & $<0.02$ & 5.9 & $<0.02$ & 0.05 & 1,1 & 1.6 & $<0.004$ \\
\hline SMTF-6C & 0.50 & 0.5 & $<0.1$ & $<0.2$ & 0.01 & 0.01 & $<0.02$ & 1.6 & $<0.02$ & $<0.01$ & $<0.2$ & 0.6 & $<0.004$ \\
\hline SMTF-6D & 0.25 & $<0.4$ & $<0.1$ & $<0.2$ & $<0.004$ & $<0.004$ & $<0.02$ & $<0.4$ & $<0,02$ & $<0.01$ & $<0.2$ & 0.6 & $<0.004$ \\
\hline SMTF-GE & $<0.25$ & $<0.4$ & $<0.1$ & $<0.2$ & 0.01 & 0.00 & $<0.02$ & $<0.4$ & $<0.02$ & $<0.01$ & $<0.2$ & 0.3 & $<0.004$ \\
\hline SMTF-6 & Total & 6.1 & $<0.1$ & $<0.2$ & 0.07 & 0.22 & $<0.02$ & 27,3 & $<0.02$ & 0.26 & 5.7 & 8.7 & $<0.004$ \\
\hline SMWF-1A & 2.5 & 1.8 & $<0.1$ & $<0.2$ & 0.01 & 0.12 & $<0.02$ & 8.0 & 0.04 & 0.31 & 3.1 & 4.9 & $<0.004$ \\
\hline SMWF-1B & 1.0 & 0.6 & $<0.1$ & $<0.2$ & $<0.004$ & 0.03 & $<0.02$ & 1.6 & $<0.02$ & 0.06 & 0.6 & 1.3 & $<0.004$ \\
\hline SMWF-1C & 0.50 & 0.4 & $<0.1$ & $<0.2$ & $<0.004$ & 0.01 & $<0.02$ & $<0.4$ & $<0.02$ & 0.01 & $<0.2$ & 0.4 & $<0.004$ \\
\hline SMWF-ID & 0.25 & 0.4 & $<0.1$ & $<0.2$ & $<0.004$ & $<0.004$ & $<0.02$ & $<0.4$ & 0.02 & $<0.01$ & $<0.2$ & 0.3 & $<0.004$ \\
\hline SMWF-1E & $<0.25$ & 1.1 & $<0.1$ & $<0.2$ & 0.08 & 0.01 & $<0.02$ & 185.4 & 0.02 & $<0.01$ & 0.2 & 0.8 & $<0.004$ \\
\hline SMWF-1 & Total & 4.4 & $<0.1$ & $<0.2$ & 0.10 & 0.17 & $<0.02$ & 194.9 & 0.09 & 0.38 & 3.9 & 7.7 & $<0.004$ \\
\hline SMWF-2A & 2.5 & 20.2 & $<0.1$ & $<0.2$ & 0.05 & 1.08 & $<0.02$ & 13.3 & 0.13 & 0.37 & 45.7 & 12.1 & 0.006 \\
\hline SMWF-2B & 1.0 & 7.5 & $<0.1$ & $<0.2$ & 0.02 & 0.29 & $<0.02$ & 3.7 & 0.05 & 0.08 & 11.4 & 3.9 & $<0.004$ \\
\hline SMWF-2C & 0.50 & 2.5 & $<0.1$ & $<0.2$ & 0.00 & 0.06 & $<0.02$ & 0.9 & 0.03 & 0.02 & 2.0 & 1.0 & $<0.004$ \\
\hline SMWF-2D & 0.25 & 0.9 & $<0.1$ & $<0.2$ & $<0.004$ & 0.02 & $<0.02$ & $<0.4$ & $<0.02$ & $<0.01$ & 0.5 & 0.4 & $<0.004$ \\
\hline SMWF-2E & $<0.25$ & 1.6 & $<0.1$ & $<0.2$ & 0.08 & 0.02 & $<0.02$ & 172.5 & 0.03 & $<0.01$ & 0.8 & 0.5 & $<0.004$ \\
\hline SMWF-2 & Total & 32.6 & $<0.1$ & $<0.2$ & 0.16 & 1.48 & $<0.02$ & 190.4 & 0.23 & 0.47 & 60.4 & 17.9 & 0.006 \\
\hline
\end{tabular}




\begin{tabular}{|c|c|c|c|c|c|c|c|c|c|c|c|c|c|}
\hline \multicolumn{14}{|c|}{$\begin{array}{l}\text { FACILITY \#1 - Copper/Beryllium Foundry and Machine Shop } \\
\text { Sioutas Cascade Impactor, Size-Selective Sample Results for Thirty-one Elements }\end{array}$} \\
\hline $\begin{array}{c}\text { Sample } \\
\text { Number }\end{array}$ & $\begin{array}{l}\text { Particle } \\
\text { Stze } \\
(\mu \mathrm{m})\end{array}$ & $\begin{array}{c}\text { Al } \\
\text { Conc, } \\
\left(\mu g / \mathrm{m}^{3}\right)\end{array}$ & $\begin{array}{c}\text { Sb } \\
\text { Conc. } \\
\left(\mu g / m^{3}\right)\end{array}$ & $\begin{array}{c}\text { As } \\
\text { Conc. } \\
\left(\mu g / \mathrm{m}^{3}\right)\end{array}$ & $\begin{array}{l}\text { Ba } \\
\text { Conc. } \\
\left(\mu g / \mathrm{m}^{3}\right)\end{array}$ & $\begin{array}{c}\mathrm{Be} \\
\text { Conc. } \\
\left(\mu \mathrm{g} / \mathrm{m}^{3}\right)\end{array}$ & $\begin{array}{c}\text { Cd } \\
\text { Conc. } \\
\left(\mu g / m^{3}\right)\end{array}$ & $\begin{array}{c}\text { Ce } \\
\text { Conc. } \\
\left(\mu g / m^{3}\right)\end{array}$ & $\begin{array}{c}\mathrm{Cr} \\
\text { Conc. } \\
\left(\mu \mathrm{g} / \mathrm{m}^{3}\right)\end{array}$ & $\begin{array}{c}\text { Co } \\
\text { Conc. } \\
\left(\mu g / \mathrm{m}^{3}\right)\end{array}$ & $\begin{array}{c}\mathrm{Cu} \\
\mathrm{Conc} \\
\left(\mathrm{\mu g} / \mathrm{m}^{3}\right)\end{array}$ & $\begin{array}{c}F \boldsymbol{G} \\
\text { Conc. } \\
\left(\mu g / \mathrm{m}^{3}\right)\end{array}$ & $\begin{array}{c}\text { La } \\
\text { Conc. } \\
\left(\mu \mathrm{g} / \mathrm{m}^{3}\right)\end{array}$ \\
\hline SMWF-3A & 2.5 & 12.5 & $<0.1$ & $\leq 0.2$ & 0.05 & 1.60 & $<0.02$ & 17.7 & 0.13 & 0.73 & 22.9 & 9.0 & $<0.004$ \\
\hline SMWF-3B & 1.0 & 2.9 & $<0.1$ & $<0.2$ & 0.01 & 0.37 & $<0.02$ & 2.9 & 0.02 & 0.12 & 4.2 & 1.7 & $<0.004$ \\
\hline SMWF-3C & 0.50 & 0.8 & $<0.1$ & $<0.2$ & $<0.004$ & 0.11 & $<0.02$ & 0.5 & $<0.02$ & 0.03 & 1.1 & 0.4 & $<0.004$ \\
\hline SMWF-3D & 0.25 & 1.1 & $<0.1$ & $<0.2$ & $<0.004$ & 0.11 & $<0.02$ & $<0.4$ & 0.03 & 0.02 & 0.7 & 0.2 & $<0.004$ \\
\hline SMWF-3E & $<0.25$ & $<0.4$ & $<0.1$ & $<0.2$ & $<0.004$ & 0.06 & 0.02 & $<0.4$ & 0.03 & $<0.01$ & 0.9 & $<0.1$ & $<0.004$ \\
\hline SMWF-3 & Total & 17.3 & $<0.1$ & $<0.2$ & 0.06 & 2.26 & 0.02 & 21.1 & 0.21 & 0.90 & 29.7 & 11.3 & $<0.004$ \\
\hline SMWF-4A & 2.5 & 20.3 & $<0.1$ & $<0.2$ & 0.07 & 0.87 & $<0.02$ & 20.3 & 0.11 & 5.48 & 38.6 & 14.4 & 0.010 \\
\hline SMWF-4C & 0.50 & 2.2 & $<0.1$ & $<0.2$ & 0.01 & 0.06 & $<0.02$ & 1.8 & $<0.02$ & 0.22 & 2.4 & 1.2 & $<0.004$ \\
\hline SMWF-4D & 0.25 & 0.6 & $<0.1$ & $<0.2$ & $<0.004$ & 0.02 & $<0.02$ & $<0.4$ & $<0.02$ & 0.04 & 0.4 & 0.3 & $<0.004$ \\
\hline SMWF-4E & $<0.25$ & 1.2 & $<0.1$ & $<0.2$ & 0.07 & 0.02 & $<0.02$ & 176.7 & $<0.02$ & 0.04 & 0.6 & 0.5 & $<0.004$ \\
\hline SMWF-4 & Total & 30.9 & $<0.1$ & $<0.2$ & 0.17 & 1.20 & $<0.02$ & 204.1 & 0.15 & 6.82 & 50.6 & 20.2 & 0.010 \\
\hline SMWF-5A & 2.5 & 12.1 & $<0.1$ & $<0.2$ & 0.20 & 6.05 & $<0.02$ & 16.9 & 0.25 & 1.46 & 229.6 & 17.3 & 0.005 \\
\hline SMWF-5B & 1.0 & 3.3 & $<0.1$ & $<0,2$ & 0.05 & 1.79 & $<0.02$ & 4.4 & 0.06 & 0.27 & 62.6 & 5.0 & $<0.004$ \\
\hline SMWF-5C & 0.50 & 1.2 & $<0.1$ & $<0.2$ & 0.01 & 0.65 & $<0.02$ & 1.2 & 0.03 & 0.09 & 23.0 & 1.5 & $<0.004$ \\
\hline SMWF-5D & 0.25 & 0.8 & $<0.1$ & $<0.2$ & 0.01 & 0.23 & $<0.02$ & 0.5 & $<0.02$ & 0.03 & 7.5 & 0.7 & $<0.004$ \\
\hline SMWF-5E & $<0.25$ & 1.4 & $<0.1$ & $<0.2$ & 0.09 & 0.25 & $<0.02$ & 194.1 & 0.02 & 0.04 & 8.8 & 1.1 & $<0.004$ \\
\hline SMWF-5 & Total & 18.8 & $<0.1$ & $<0.2$ & 0.36 & 8.97 & $<0.02$ & 217.1 & 0.36 & 1.90 & 331.4 & 25.7 & 0.005 \\
\hline SMWF-6A & 2.5 & 6.5 & $<0.1$ & $<0.2$ & 0.04 & 0.33 & 0.16 & 8.8 & 0.12 & 0.23 & 11.3 & 5.2 & 0.026 \\
\hline SMWF-6B & 1.0 & 2.7 & $<0.1$ & $<0.2$ & 0.03 & 0.10 & 0.12 & 2.1 & 0.04 & 0.06 & 3.3 & 1.5 & 0.004 \\
\hline SMWF-6E & $<0.25$ & 1.6 & $<0,1$ & $<0.2$ & 0.08 & 0.05 & $<0.02$ & 192.1 & $<0.02$ & 0.02 & 2.7 & 0.5 & $<0.004$ \\
\hline SMWF-6 & Total & 13.0 & $<0.1$ & $<0.2$ & 0.18 & 0.54 & 0.33 & 203.0 & 0.16 & 0.33 & 18.8 & 7.8 & 0.029 \\
\hline
\end{tabular}




\begin{tabular}{|c|c|c|c|c|c|c|c|c|c|c|c|c|c|}
\hline \multicolumn{14}{|c|}{$\begin{array}{l}\text { FACILITY \#1 - Copper/Beryllium Foundry and Machine Shop } \\
\text { Sioutas Cascade Impactor, Size-Selective Sample Results for Thirty-one Elements }\end{array}$} \\
\hline $\begin{array}{l}\text { Sample } \\
\text { Number } \\
\end{array}$ & $\begin{array}{l}\text { Particle } \\
\text { Slze } \\
(\mu \mathrm{m})\end{array}$ & $\begin{array}{c}\text { Al } \\
\text { Conc. } \\
\left(\mu g / \mathrm{m}^{3}\right)\end{array}$ & $\begin{array}{c}\text { Sb } \\
\text { Conc. } \\
\left(\mu g / m^{3}\right)\end{array}$ & $\begin{array}{c}\text { As } \\
\text { Conc. } \\
\left(\mu g / m^{3}\right)\end{array}$ & $\begin{array}{c}\text { Ba } \\
\text { Conc. } \\
\left(\mu g / m^{3}\right)\end{array}$ & $\begin{array}{c}\mathrm{Be} \\
\mathrm{Conc} \\
\left(\mu \mathrm{g} / \mathrm{m}^{3}\right) \\
\end{array}$ & $\begin{array}{c}\text { Cd } \\
\text { Conc. } \\
\left(\mu \mathrm{g} / \mathrm{m}^{3}\right)\end{array}$ & $\begin{array}{c}\mathrm{Ca} \\
\text { Conc. } \\
\left(\mathrm{\mu g} / \mathrm{m}^{3}\right)\end{array}$ & $\begin{array}{c}\text { Cr } \\
\text { Conc. } \\
\left(\mu g / \mathrm{m}^{3}\right)\end{array}$ & $\begin{array}{c}\text { Co } \\
\text { Conc. } \\
\left(\mu g / \mathrm{m}^{3}\right)\end{array}$ & $\begin{array}{c}\mathrm{Cu} \\
\mathrm{Conc} \\
\left(\mu \mathrm{g} / \mathrm{m}^{3}\right)\end{array}$ & $\begin{array}{c}F_{\theta} \\
\text { Conc. } \\
\left(\mu g / m^{3}\right)\end{array}$ & $\begin{array}{c}\text { La } \\
\text { Conc. } \\
\left(\mu g / \mathrm{m}^{3}\right)\end{array}$ \\
\hline SMWF-7A & 2.5 & 3.6 & $<0.1$ & $<0.2$ & 0.02 & 2.57 & $<0.02$ & 5.6 & 0.30 & 0.84 & 23.6 & 4.7 & $<0.004$ \\
\hline SMWF-7B & 1.0 & 0.9 & $<0.1$ & $<0.2$ & 0.01 & 0.56 & $<0.02$ & 0.9 & 0.05 & 0.15 & 4.9 & 0.9 & $<0.004$ \\
\hline SMWF-7C & 0.50 & $<0.4$ & $<0.1$ & $<0.2$ & $<0.004$ & 0.16 & $<0.02$ & $<0.4$ & $<0.02$ & 0.03 & 1.0 & 0.2 & $<0.004$ \\
\hline SMWF-7D & 0.25 & 0.5 & $<0.1$ & $<0.2$ & $<0.004$ & 0.12 & 0.03 & $<0.4$ & $<0.02$ & $<0.01$ & 0.7 & 0.3 & $<0.004$ \\
\hline SMWF-7E & $<0.25$ & 1.1 & $<0.1$ & $<0.2$ & 0.10 & 0.26 & $<0.02$ & 235.6 & 0.02 & 0.03 & 1.5 & 0.4 & $<0.004$ \\
\hline SMWF-7 & Total & 6.2 & $<0.1$ & $<0.2$ & 0.12 & 3.67 & 0.03 & 242.0 & 0.37 & 1.05 & 31.7 & 6.6 & $<0.004$ \\
\hline SMWF-8A & 2.5 & $<0.5$ & $<0.1$ & $<0,3$ & $<0.005$ & 0.04 & $<0.02$ & 2.9 & $<0.02$ & 0.03 & 1.7 & 0.9 & $<0.005$ \\
\hline SMWF-BB & 1.0 & $<0.5$ & $<0.1$ & $<0.3$ & $<0.005$ & 0.01 & $<0.02$ & $<0.5$ & $<0.02$ & $<0.01$ & 0.3 & 0.2 & $<0.005$ \\
\hline SMWF-8C & 0.50 & $<0.5$ & $<0.1$ & $<0,3$ & $<0.005$ & $<0.005$ & $<0.02$ & $<0.5$ & $<0.02$ & $<0.01$ & $<0.2$ & $<0.1$ & $<0.005$ \\
\hline SMWF-8D & 0.25 & $<0.5$ & $<0.1$ & $<0.3$ & $<0.005$ & $<0.005$ & $<0.02$ & $<0.5$ & $<0.02$ & $<0.01$ & $<0.2$ & $<0.1$ & $<0.005$ \\
\hline SMWF-BE & $<0.25$ & 0.8 & $<0.1$ & $<0.3$ & 0.10 & $<0.005$ & $<0.02$ & 240.4 & $<0.02$ & $<0.01$ & $<0.2$ & 0.3 & $<0.005$ \\
\hline SMWF-8 & Total & 0.8 & $<0.1$ & $<0.3$ & 0.10 & 0.05 & $<0.02$ & 243.3 & $<0.02$ & 0.03 & 2.0 & 1.4 & $<0.005$ \\
\hline
\end{tabular}




\section{Appendix C-continued}

FACILITY \#1 - Copper/Beryllium Foundry and Machine Shop

Sioutas Cascade Impactor, Size-Selective Sample Results for Thirty-one Elements

\begin{tabular}{|c|c|c|c|c|c|c|c|c|c|c|c|c|c|}
\hline $\begin{array}{c}\text { Sample } \\
\text { Number }\end{array}$ & $\begin{array}{c}\text { Partlcle } \\
\text { Size } \\
(\mu \mathrm{m}) \\
\end{array}$ & $\begin{array}{c}\mathrm{Pb} \\
\text { Conc. } \\
\left(\mu g / m^{3}\right)\end{array}$ & $\begin{array}{c}\mathrm{LI} \\
\mathrm{Conc} \\
\left(\mu \mathrm{g} / \mathrm{m}^{3}\right)\end{array}$ & $\begin{array}{c}\mathrm{Mg} \\
\mathrm{Conc} \\
\left(\mu \mathrm{g} / \mathrm{m}^{3}\right)\end{array}$ & $\begin{array}{c}\text { Mn } \\
\text { Conc. } \\
\left(\mu \mathrm{g} / \mathrm{m}^{3}\right)\end{array}$ & $\begin{array}{c}\text { Mo } \\
\text { Conc. } \\
\left(\mu g / m^{2}\right)\end{array}$ & $\begin{array}{c}\text { NI } \\
\text { Conc. } \\
\left(\mu \mathrm{g} / \mathrm{m}^{3}\right)\end{array}$ & $\begin{array}{c}\text { P } \\
\text { Conc. } \\
\left\langle\mu g / m^{3}\right)\end{array}$ & $\begin{array}{c}K \\
\text { Conc. } \\
\left(\mu g / m^{3}\right)\end{array}$ & $\begin{array}{c}5 \theta \\
\text { Conc. } \\
\left(\mu g / \mathrm{m}^{3}\right)\end{array}$ & $\begin{array}{c}\mathrm{Ag} \\
\text { Conc. } \\
\left(\mu \mathrm{g} / \mathrm{m}^{3}\right)\end{array}$ & $\begin{array}{c}S r \\
\text { Conc. } \\
\left(\mu \mathrm{g} / \mathrm{m}^{3}\right)\end{array}$ & $\begin{array}{c}\text { Te } \\
\text { Conc. } \\
\left(\mu \mathrm{g} / \mathrm{m}^{3}\right)\end{array}$ \\
\hline SMTF-1A & 2.5 & 4.0 & $<0.2$ & 18.7 & 0.24 & 0.03 & 0.46 & $<0,2$ & 1.4 & $<1$ & 0.008 & 0.08 & $<0.01$ \\
\hline SMTF-1B & 1.0 & 5.2 & $<0.2$ & 1.2 & 0.01 & $<0.02$ & $<0.02$ & $<0.2$ & $<0 . \underline{2}$ & $<1$ & $<0.002$ & 0.01 & $<0.01$ \\
\hline SMTF-1C & 0.50 & 5.8 & $<0.2$ & $<0.1$ & $<0.01$ & $<0.02$ & $<0.02$ & $<0.2$ & $<0.2$ & $<1$ & $<0.002$ & $<0.004$ & $<0.01$ \\
\hline SMTF-1D & 0.25 & 4.6 & $<0.2$ & $<0.1$ & $<0.01$ & $<0.02$ & $<0.02$ & $<0.2$ & $<0.2$ & $<1$ & $<0.002$ & $<0.004$ & $<0.01$ \\
\hline SMTF-1E & $<0.25$ & $<0.1$ & $<0.2$ & $<0.1$ & $<0.01$ & $<0.02$ & $<0.02$ & $<0.2$ & 0.2 & $<1$ & $<0.002$ & $<0.004$ & $<0.01$ \\
\hline SMTF-1 & Total & 19.7 & $<0.2$ & 19.9 & 0.25 & 0.03 & 0.46 & $<0.2$ & 1.6 & $<1$ & 0.008 & 0.08 & $<0.01$ \\
\hline
\end{tabular}

\begin{tabular}{|c|c|c|c|c|c|c|c|c|c|c|c|c|c|}
\hline SMTF-2A & 2.5 & 7.0 & $<0.2$ & 7.6 & 0.39 & 0.02 & 0.25 & $<0.2$ & 0.9 & $<1$ & 0.002 & 0.05 & $<0.01$ \\
\hline SMTF-2B & 1.0 & 2.1 & $<0.2$ & 2.0 & 0.08 & $<0.02$ & 0.06 & $<0.2$ & 0.3 & $<1$ & $<0.002$ & 0.01 & $<0.01$ \\
\hline SMTF-2C & 0.50 & 4.9 & $<0.2$ & 0.4 & 0.01 & $<0.02$ & $<0.02$ & $<0.2$ & $<0.2$ & $<1$ & $<0.002$ & $<0.004$ & $<0.01$ \\
\hline SMTF-2D & 0.25 & 1.2 & $<0.2$ & $<0.1$ & $<0.01$ & $<0.02$ & $<0.02$ & $<0.2$ & $<0.2$ & $<1$ & $<0.002$ & $<0.004$ & $<0.01$ \\
\hline SMTF-2E & $<0.25$ & $<0.1$ & $<0.2$ & 0.1 & 0.01 & $<0.02$ & $<0.02$ & $<0.2$ & 0.3 & $<1$ & 0.004 & $<0.004$ & $<0.01$ \\
\hline SMTF-2 & Total & 15.2 & $<0.2$ & 10.1 & 0.49 & 0.02 & 0.31 & $<0.2$ & 1.5 & $<1$ & 0.006 & 0.06 & $<0.01$ \\
\hline
\end{tabular}

\begin{tabular}{|c|c|c|c|c|c|c|c|c|c|c|c|c|c|}
\hline SMTF-3A & 2.5 & 4.3 & $<0.3$ & 7.3 & 0.19 & $<0.03$ & 0.53 & $<0.3$ & 1.2 & $<1$ & 0.016 & 0.06 & $<0.01$ \\
\hline SMTF-3B & 1.0 & 5.0 & $<0.3$ & $<0.2$ & $<0.01$ & $<0.03$ & $<0.03$ & $<0.3$ & $<0.3$ & $<1$ & $<0.003$ & $<0.007$ & $<0.01$ \\
\hline SMTF-3C & 0.50 & 1.8 & $<0.3$ & $<0.2$ & $<0.01$ & $<0.03$ & $<0.03$ & $<0.3$ & $<0.3$ & $<1$ & $<0.003$ & $<0.007$ & $<0.01$ \\
\hline SMTF-3D & 0.25 & 4.0 & $<0.3$ & $<0.2$ & $<0.01$ & $<0.03$ & $<0.03$ & $<0.3$ & $<0.3$ & $<1$ & $<0.003$ & $<0.007$ & $<0.01$ \\
\hline SMTF-3E & $<0.25$ & $<0.1$ & $<0.3$ & $<0.2$ & 0.01 & $<0.03$ & $<0.03$ & $<0.3$ & $<0.3$ & $<1$ & $<0.003$ & $<0.007$ & $<0.01$ \\
\hline SMTF 3 & Total & 15.2 & $<0.3$ & 7.3 & 0.20 & $<0.03$ & 0.53 & $<0.3$ & 1.2 & $<1$ & 0.016 & 0.06 & $<0.01$ \\
\hline
\end{tabular}

\begin{tabular}{|c|c|c|c|c|c|c|c|c|c|c|c|c|c|}
\hline SMTF-4A & 2.5 & 2.2 & $<0.2$ & 13.1 & 0.09 & $<0.02$ & 0.38 & $<0.2$ & 0.6 & $<1$ & 0.005 & 0.03 & $<0.01$ \\
\hline SMTF-4B & 1.0 & 1.5 & $<0.2$ & 3.8 & 0.02 & $<0.02$ & 0.14 & $<0.2$ & 0.3 & $<1$ & $<0.002$ & 0.01 & $\leq 0.01$ \\
\hline SMTF-4C & 0.50 & 5.2 & $<0.2$ & 1.2 & $<0.01$ & $<0.02$ & 0.05 & $<0.2$ & $<0.2$ & $<1$ & $<0.002$ & $<0.004$ & $<0.01$ \\
\hline SMTF-4D & 0.25 & 1.8 & $<0.2$ & 0.3 & $<0.01$ & $<0.02$ & $<0.02$ & $<0.2$ & $<0.2$ & $<1$ & $<0.002$ & $<0.004$ & $<0.01$ \\
\hline SMTF-4E & $<0.25$ & $<0.1$ & $<0.2$ & 0.5 & 0.02 & $<0.02$ & 0.02 & $<0.2$ & $<0.2$ & $<1$ & 0.008 & 0.01 & $<0.01$ \\
\hline SMTF-4 & Total & 10.8 & $<0.2$ & 18.9 & 0.13 & $<0.02$ & 0.59 & $<0.2$ & 0.9 & $<1$ & 0.013 & 0.05 & $<0.01$ \\
\hline
\end{tabular}




\begin{tabular}{|c|c|c|c|c|c|c|c|c|c|c|c|c|c|}
\hline \multicolumn{14}{|c|}{$\begin{array}{l}\text { FACILITY \#1 - Copper/Beryllium Foundry and Machine Shop } \\
\text { Sioutas Cascade Impactor, Size-Selective Sample Results for Thirty-one Elements }\end{array}$} \\
\hline $\begin{array}{l}\text { Sample } \\
\text { Number }\end{array}$ & $\begin{array}{l}\text { Particle } \\
\text { Slze } \\
(\mu m)\end{array}$ & $\begin{array}{c}\text { Pb } \\
\text { Conc. } \\
\left(\mu g / \mathrm{m}^{3}\right)\end{array}$ & $\begin{array}{l}\text { LI } \\
\text { Conc. } \\
\left(\mu g / m^{3}\right)\end{array}$ & $\begin{array}{c}\mathrm{Mg} \\
\text { Conc. } \\
\left(\mu g / \mathrm{m}^{2}\right)\end{array}$ & $\underset{\text { Conc. }}{\operatorname{Mn}}$ & $\begin{array}{c}\text { Mo } \\
\text { Conc. } \\
\left(\mathrm{Hg} / \mathrm{m}^{3}\right)\end{array}$ & $\begin{array}{c}\text { NI } \\
\text { Conc. } \\
\left(\mu g / \mathrm{m}^{3}\right)\end{array}$ & $\begin{array}{c}\text { P } \\
\text { Conc. } \\
\left(\mu g / m^{3}\right)\end{array}$ & $\begin{array}{c}K \\
\text { Conc. } \\
\left(\mu g / m^{3}\right)\end{array}$ & $\begin{array}{c}\text { Se } \\
\text { Conc. } \\
\left(\mu g / m^{3}\right)\end{array}$ & $\begin{array}{c}\mathrm{Ag} \\
\text { Conc. } \\
\left(\mu \mathrm{g} / \mathrm{m}^{3}\right)\end{array}$ & $\begin{array}{c}\mathrm{Sr} \\
\text { Conc. } \\
\left(\mu \mathrm{g} / \mathrm{m}^{2}\right)\end{array}$ & $\begin{array}{c}\text { Te } \\
\text { Conc. } \\
\left(\mu \mathrm{g} / \mathrm{m}^{3}\right) \\
\end{array}$ \\
\hline SMTF-5A & 2.5 & 0.4 & $<0.2$ & 5.7 & 0.09 & $<0.02$ & 0.06 & $<0.2$ & 0.6 & $<1$ & $<0.002$ & 0.03 & $<0.01$ \\
\hline SMTF-5B & 1.0 & 1.1 & $<0.2$ & 1.0 & 0.01 & $<0.02$ & $<0.02$ & $<0.2$ & $<0.2$ & $<1$ & $<0.002$ & 0.01 & $<0.01$ \\
\hline SMTF-5C & 0.50 & 1.0 & $<0.2$ & 0.2 & $<0.01$ & $<0.02$ & $<0.02$ & $<0.2$ & $<0.2$ & $<1$ & $<0.002$ & $<0.004$ & $<0.01$ \\
\hline SMTF-5D & 0.25 & 5.7 & $<0.2$ & 0.2 & $<0.01$ & $<0.02$ & $<0.02$ & $<0.2$ & $<0.2$ & $<1$ & $<0.002$ & $<0.004$ & $<0.01$ \\
\hline SMTF-5E & $<0.25$ & $<0.1$ & $<0.2$ & $<0.1$ & $<0.01$ & $<0.02$ & $<0.02$ & $<0.2$ & $<0.2$ & $<1$ & $<0.002$ & $<0.004$ & $<0.01$ \\
\hline SMTF-5 & Total & 8.1 & $<0.2$ & 7.1 & 0.10 & $<0.02$ & $<0.02$ & $<0.2$ & $<0.2$ & $<1$ & $<0.002$ & 0.04 & $<0.01$ \\
\hline SMTF-6A & 2.5 & 0.3 & $<0.2$ & 11.1 & 0.15 & $<0.02$ & 0.06 & $<0.2$ & 0.7 & $<1$ & $<0.002$ & 0.06 & $<0.01$ \\
\hline SMTF-6B & 1.0 & 0.2 & $<0.2$ & 3.6 & 0.05 & $<0.02$ & $<0.02$ & $<0.2$ & 0.2 & $<1$ & $<0.002$ & 0.02 & $<0.01$ \\
\hline SMTF-6C & 0.50 & 0.2 & $<0.2$ & 1.1 & $<0.01$ & $<0.02$ & $<0.02$ & $<0.2$ & $<0.2$ & $<1$ & $<0.002$ & 0.00 & $<0.01$ \\
\hline SMTF-6D & 0.25 & 0.3 & $<0.2$ & 0.2 & $<0.01$ & $<0.02$ & $<0.02$ & $<0.2$ & $<0.2$ & $<1$ & $<0.002$ & $<0.004$ & $<0.01$ \\
\hline SMTF-6E & $<0.25$ & $<0.1$ & $<0.2$ & 0.2 & 0.01 & $<0.02$ & $<0.02$ & $<0.2$ & $<0.2$ & $<1$ & 0.004 & $<0.004$ & $<0.01$ \\
\hline SMTF-6 & Total & 0.9 & $<0.2$ & 16.1 & 0.21 & $<0.02$ & 0.06 & $<0.2$ & 0.9 & $<1$ & 0.004 & 0.08 & $<0.01$ \\
\hline SMWF-1A & 2.5 & 1.2 & $<0.2$ & 2.5 & 0.07 & $<0.02$ & 0.04 & $<0.2$ & 0.3 & $<1$ & 0.002 & 0.02 & $<0.01$ \\
\hline SMWF-1B & 1.0 & 1.0 & $<0.2$ & 0.6 & 0.01 & $<0.02$ & $<0.02$ & $<0.2$ & $<0.2$ & $<1$ & $<0.002$ & $<0.004$ & $<0.01$ \\
\hline SMWF-1C & 0.50 & 5.5 & $<0.2$ & 0.2 & $<0.01$ & $<0.02$ & $<0.02$ & $<0.2$ & $<0.2$ & $<1$ & $<0.002$ & $<0.004$ & $<0.01$ \\
\hline SMWF-1D & 0.25 & 3.7 & $<0.2$ & $<0.1$ & $<0.01$ & $<0.02$ & $<0.02$ & $<0.2$ & $<0.2$ & $<1$ & $<0.002$ & $<0.004$ & $<0.01$ \\
\hline SMWF-1E & $<0.25$ & $<0.1$ & $<0.2$ & 1.4 & 0.02 & $<0.02$ & $<0.02$ & 107 & 0.6 & $<1$ & $<0.002$ & 0.13 & $<0.01$ \\
\hline SMWF-1 & Total & 11.4 & $<0,2$ & 4.6 & 0.10 & $<0.02$ & 0.04 & 107 & 0.9 & $<1$ & 0.002 & 0.15 & $<0.01$ \\
\hline SMWF-2A & 2.5 & 1.2 & $<0.2$ & 6.2 & 0.17 & $<0.02$ & 0.23 & $<0.2$ & 0.7 & $\leq 1$ & 0.011 & 0.08 & 0.08311 \\
\hline SMWF-2B & 1.0 & 1.5 & $<0.2$ & 1.8 & 0.05 & 0.02 & 0.05 & $<0.2$ & 0.2 & $<1$ & $<0.002$ & 0.02 & 0.08311 \\
\hline SMWF-2C & 0.50 & 2.5 & $<0.2$ & 0.4 & $<0.01$ & $<0.02$ & $<0.02$ & $<0.2$ & $<0.2$ & $<1$ & $<0.002$ & 0.01 & 0.08311 \\
\hline SMWF-2D & 0.25 & 0.6 & $<0.2$ & $<0.1$ & $<0.01$ & $<0.02$ & $<0.02$ & $<0.2$ & $<0.2$ & $<1$ & $<0.002$ & $<0.004$ & 0.08311 \\
\hline SMWF-2E & $<0.25$ & $<0.1$ & $<0.2$ & 1.0 & 0.03 & $<0.02$ & $<0.02$ & 87 & 0.4 & $<1$ & $<0.002$ & 0.12 & 0.09 \\
\hline SMWF-2 & Total & 5.8 & $<0.2$ & 9.5 & 0.25 & 0.02 & 0.28 & 87 & 1.4 & $<1$ & 0.011 & 0.22 & 0.09 \\
\hline
\end{tabular}




\begin{tabular}{|c|c|c|c|c|c|c|c|c|c|c|c|c|c|}
\hline \multicolumn{14}{|c|}{$\begin{array}{l}\text { FACILITY \#1 - Copper/Beryllium Foundry and Machine Shop } \\
\text { Sioutas Cascade Impactor, Size-Selective Sample Results for Thirty-one Elements }\end{array}$} \\
\hline $\begin{array}{l}\text { Sample } \\
\text { Number }\end{array}$ & $\begin{array}{l}\text { Partlcle } \\
\text { Size } \\
(\mu \mathrm{m})\end{array}$ & $\begin{array}{c}\text { Pb } \\
\text { Conc. } \\
\left(\mu g / \mathrm{m}^{3}\right)\end{array}$ & $\begin{array}{l}\text { Lunc. } \\
\text { Cog/mi }\end{array}$ & $\begin{array}{c}\text { Mg } \\
\text { Conc. } \\
\left(\mu g / m^{3}\right)\end{array}$ & $\begin{array}{c}\text { Mn } \\
\text { Conc. } \\
\left(\mu \mathrm{g} / \mathrm{m}^{3}\right)\end{array}$ & $\begin{array}{c}\text { Mo } \\
\text { Conc. } \\
\left(\mu \mathrm{g} / \mathrm{m}^{3}\right)\end{array}$ & $\begin{array}{c}\text { N! } \\
\text { Conc. } \\
\left(\mu \mathrm{g} / \mathrm{m}^{3}\right)\end{array}$ & $\begin{array}{c}P \\
\text { Conc. } \\
\left(\mu \mathrm{g} / \mathrm{m}^{3}\right)\end{array}$ & $\begin{array}{c}\mathrm{K} \\
\text { Conc. } \\
\left(\mu \mathrm{g} / \mathrm{m}^{3}\right)\end{array}$ & $\begin{array}{c}\text { Se } \\
\text { Conc. } \\
\left(\mu \mathrm{g} / \mathrm{m}^{3}\right)\end{array}$ & $\begin{array}{c}\mathrm{Ag} \\
\text { Conc. } \\
\left(\mu \mathrm{g} / \mathrm{m}^{3}\right)\end{array}$ & $\begin{array}{c}\text { Sr } \\
\text { Conc. } \\
\left(\mu g / \mathrm{m}^{3}\right)\end{array}$ & $\begin{array}{c}\text { Te } \\
\text { Conc. } \\
\left.\text { (ug/m } / m^{3}\right)\end{array}$ \\
\hline SMWF-3A & 2.5 & 0.5 & $\leq 0.2$ & 7.5 & 0.20 & 0.02 & 0.40 & $<0.2$ & 0.9 & $<1$ & 0.007 & 0.05 & $<0.01$ \\
\hline SMWF-3B & 1.0 & 0.5 & $<0.2$ & 1.2 & 0.03 & $<0.02$ & 0.07 & $<0.2$ & $<0.2$ & $<1$ & $<0.002$ & 0.01 & $<0.01$ \\
\hline SMWF-3C & 0.50 & 0.6 & $<0.2$ & 0.3 & $<0.01$ & $<0.02$ & $<0.02$ & $<0.2$ & $<0.2$ & $<1$ & $<0.002$ & $<0.004$ & $<0.01$ \\
\hline SMWF-3D & 0.25 & 0.9 & $<0.2$ & 0.1 & $<0.01$ & $<0.02$ & $<0.02$ & $<0.2$ & $<0.2$ & $<1$ & $<0.002$ & $<0.004$ & $<0.01$ \\
\hline SMWF-3E & $<0.25$ & $<0.1$ & $<0.2$ & $<0.1$ & $<0.01$ & $<0.02$ & $<0.02$ & $<0.2$ & $<0.2$ & $<1$ & $<0.002$ & $<0.004$ & $<0.01$ \\
\hline SMWF-3 & Total & 2.4 & $<0.2$ & 9.1 & 0.23 & 0.02 & 0.47 & $<0.2$ & 0.9 & $<1$ & 0.007 & 0.05 & $<0.01$ \\
\hline SMWF-4A & 2.5 & 2.6 & $<0.2$ & 6.9 & 0.69 & $<0.02$ & 0.30 & $<0.2$ & 1.1 & $<1$ & 0.004 & 0.09 & $<0.01$ \\
\hline SMWF-4B & 1.0 & 3.0 & $<0.2$ & 1.8 & 0.15 & $<0.02$ & 0.06 & $<0.2$ & 0.3 & $<1$ & $<0.002$ & 0.02 & $<0.01$ \\
\hline SMWF-4C & 0.50 & 1.2 & $<0.2$ & 0.6 & 0.04 & $<0.02$ & $<0.02$ & $<0.2$ & $<0.2$ & $<1$ & $<0.002$ & 0.01 & $<0.01$ \\
\hline SMWF-4D & 0.25 & 1.4 & $<0.2$ & 0.1 & $<0.01$ & $<0.02$ & $<0.02$ & $<0.2$ & $<0.2$ & $<1$ & $<0.002$ & $<0.004$ & $<0.01$ \\
\hline SMWF-4E & $<0.25$ & $<0.1$ & $<0.2$ & 1.1 & 0.03 & $<0.02$ & $<0.02$ & 89 & $<0.2$ & $<1$ & $<0.002$ & 0.10 & 0.09 \\
\hline SNWF-4 & Total & 8.3 & $<0.2$ & 10.6 & 0.92 & $<0.02$ & 0.36 & 89 & 1.3 & $<1$ & 0.004 & 0.22 & 0.09 \\
\hline SMWF-5A & 2.5 & 4.6 & $<0.2$ & 10.4 & 0.18 & $<0.02$ & 0.88 & $<0.2$ & 1.1 & $<1$ & 0.048 & 0.06 & $<0.01$ \\
\hline SMWF-5B & 1.0 & 0.9 & $<0.2$ & 2.5 & 0.04 & $<0.02$ & 0.15 & $<0.2$ & 0.2 & $<1$ & 0.009 & 0.02 & $<0.01$ \\
\hline SMWF-5C & 0.50 & 3.3 & $<0.2$ & 0.8 & $<0.01$ & $<0.02$ & 0.03 & $<0.2$ & $<0.2$ & $<1$ & $<0.002$ & $<0.004$ & $<0.01$ \\
\hline SMWF-5D & 0.25 & 1.6 & $<0.2$ & 0.3 & 0.01 & $<0.02$ & $<0.02$ & $<0.2$ & $<0.2$ & $<1$ & $<0.002$ & $<0.004$ & $<0.01$ \\
\hline SMWF-5E & $<0.25$ & $<0.1$ & $<0.2$ & 1.6 & 0.05 & $<0.02$ & $<0.02$ & 102 & $<0.2$ & $<1$ & $<0.002$ & 0.12 & 0.10 \\
\hline SMWF-5 & Total & 10.4 & $<0.2$ & 15.7 & 0.28 & $<0.02$ & 1.06 & 102 & 1.3 & $<1$ & 0.057 & 0.20 & 0.10 \\
\hline SMWF-6A & 2.5 & 0.9 & $<0.2$ & 5.0 & 0.07 & $<0.02$ & 0.23 & $<0.2$ & 0.4 & $\leq 1$ & $<0.002$ & 0.04 & $<0.01$ \\
\hline SMWF-6B & 1.0 & 1.3 & $\leq 0.2$ & 1.3 & 0.01 & $<0.02$ & 0.05 & $<0.2$ & $<0.2$ & $<1$ & $<0.002$ & 0.01 & 0.11 \\
\hline SMWF-6C & 0.50 & 1.8 & $<0.2$ & 0.3 & $<0.01$ & $<0.02$ & $<0.02$ & $<0.2$ & $<0.2$ & $<1$ & $<0.002$ & $<0.004$ & $<0.01$ \\
\hline SMWF-6D & 0.25 & 0.5 & $<0.2$ & 0.2 & $<0.01$ & $<0.02$ & $<0.02$ & $<0.2$ & $<0.2$ & $<1$ & $<0.002$ & $<0.004$ & $<0.01$ \\
\hline SMWF-6E & $<0.25$ & 0.1 & $<0.2$ & 1.2 & 0.02 & $<0.02$ & $<0.02$ & 108 & 0.2 & $<1$ & $<0.002$ & 0.12 & 0.11 \\
\hline SMWF-6 & Totel & 4.6 & $<0.2$ & 8.0 & 0.11 & $<0.02$ & 0.28 & 108 & 0.6 & $<1$ & $<0.002$ & 0.17 & 0.22 \\
\hline
\end{tabular}




\begin{tabular}{|c|c|c|c|c|c|c|c|c|c|c|c|c|c|}
\hline \multicolumn{14}{|c|}{$\begin{array}{l}\text { FACILITY \#1 - Copper/Beryllium Foundry and Machine Shop } \\
\text { Sioutas Cascade Impactor, Size-Selective Sample Results for Thirty-one Elements }\end{array}$} \\
\hline $\begin{array}{l}\text { Sample } \\
\text { Number }\end{array}$ & $\begin{array}{l}\text { Particle } \\
\text { Slze } \\
(\mu \mathrm{m})\end{array}$ & $\begin{array}{c}\text { Pb } \\
\text { Conc. } \\
\left(\mu g / \mathrm{m}^{3}\right)\end{array}$ & $\begin{array}{c}\text { LI } \\
\text { Conc. } \\
\left(\mu g / m^{3}\right)\end{array}$ & $\begin{array}{l}\text { Mg } \\
\text { Conc. } \\
\left(\mu g / \mathrm{m}^{3}\right)\end{array}$ & $\begin{array}{l}\text { Mn } \\
\text { Conc. } \\
\left(\mu g / \mathrm{m}^{3}\right)\end{array}$ & $\begin{array}{c}\text { Mo } \\
\text { Conc, } \\
\left(\mathrm{pg} / \mathrm{m}^{3}\right)\end{array}$ & $\begin{array}{c}\text { NI } \\
\text { Conc. } \\
\left(\mu g / m^{3}\right)\end{array}$ & $\underset{\substack{P \\
\left(\mu g / m^{2}\right)}}{c}$ & $\begin{array}{c}K \\
\text { Conc. } \\
\left(\mu g / \mathrm{m}^{3}\right)\end{array}$ & $\begin{array}{c}\text { Se } \\
\text { Conc. } \\
\left(\mu g / m^{3}\right)\end{array}$ & $\begin{array}{c}\mathrm{Ag} \\
\mathrm{Conc} \\
\left(\mu \mathrm{g} / \mathrm{m}^{3}\right)\end{array}$ & $\begin{array}{c}\text { Sr } \\
\text { Conc. } \\
\left(\mu g / \mathrm{m}^{3}\right)\end{array}$ & $\begin{array}{c}\text { Te } \\
\text { Conc } \\
\left(\mu g / \mathrm{m}^{3}\right)\end{array}$ \\
\hline SMWF-7A & 2.5 & 1.0 & $<0.2$ & 6.0 & 0.06 & $<0.02$ & 0.30 & $<0.2$ & 0.3 & $<1$ & $<0.002$ & 0.02 & $<0.01$ \\
\hline SMWF-7B & 1.0 & 0.1 & $<0.2$ & 1.1 & $<0.01$ & $<0.02$ & 0.03 & $<0.2$ & $<0.2$ & $<1$ & $<0.002$ & $<0.004$ & 0.13 \\
\hline SMWF-7C & 0.50 & 0.2 & $<0.2$ & 0.2 & $<0.01$ & $<0.02$ & $<0.02$ & $<0.2$ & $<0.2$ & $<1$ & $<0.002$ & $<0.004$ & 0.09 \\
\hline SMWF-7D & 0.25 & 1.0 & $<0.2$ & $<0.1$ & $<0.01$ & $<0.02$ & $<0.02$ & $<0.2$ & $<0.2$ & $<1$ & $<0.002$ & $<0.004$ & $<0.01$ \\
\hline SMWF-7E & $<0.25$ & 0.1 & $<0.2$ & 1.4 & 0.02 & $<0.02$ & $<0.02$ & 120 & $<0.2$ & $<1$ & $<0.002$ & 0.14 & 0.09 \\
\hline SMWF-7 & Total & 2.4 & $<0.2$ & 8.7 & 0.08 & $<0.02$ & 0.33 & 120 & 0.3 & $<1$ & $<0.002$ & 0.16 & 0.32 \\
\hline SMWF-8A & 2.5 & 0.2 & $<0.3$ & 0.9 & $<0.01$ & $<0.02$ & $<0.03$ & $<0.3$ & $<0.3$ & $<1$ & $<0.003$ & $<0.005$ & $<0.11$ \\
\hline SMWF-8B & 1.0 & 1.3 & $<0.3$ & 0.5 & $<0.01$ & $<0.02$ & $<0.03$ & $<0.3$ & $<0.3$ & $<1$ & $<0.003$ & $<0.005$ & 0.11 \\
\hline SMWF-8C & 0.50 & 1.3 & $<0.3$ & $<0.2$ & $<0.01$ & $<0.02$ & $<0.03$ & $<0.3$ & $<0.3$ & $<1$ & $<0.003$ & $<0.005$ & 0.15 \\
\hline SMWF-BD & 0.25 & 1.1 & $<0.3$ & $<0.2$ & $<0.01$ & $<0.02$ & $<0.03$ & $<0.3$ & $<0.3$ & $<1$ & $<0.003$ & 0.005342 & 0.11 \\
\hline SMWF-8E & $<0.25$ & $<0.1$ & $<0.3$ & 1.3 & 0.02 & $<0.02$ & $<0.03$ & 128 & $<0.3$ & $<1$ & $<0.003$ & 0.14 & $<0.11$ \\
\hline SMWF-8 & Total & 3.8 & $<0.3$ & 2.8 & 0.02 & $<0.02$ & $<0.03$ & 128 & $<0.3$ & $<1$ & $<0.003$ & 0.14 & 0.37 \\
\hline
\end{tabular}




\begin{tabular}{|c|c|c|c|c|c|c|c|c|}
\hline \multicolumn{9}{|c|}{$\begin{array}{l}\text { FACILITY \#1 - Copper/Beryllium Foundry and Machine Shop } \\
\text { Sioutas Cascade Impactor, Size-Selective Sample Results for Thirty-one Elements }\end{array}$} \\
\hline Sample Number & $\begin{array}{l}\text { Partlcle } \\
\text { Size } \\
(\mu \mathrm{m})\end{array}$ & $\begin{array}{l}\text { TI } \\
\text { Conc, } \\
\left(\mu g / \mathrm{m}^{3}\right)\end{array}$ & $\begin{array}{l}\text { Sn } \\
\text { Conc. } \\
\left(\mu g / \mathrm{m}^{3}\right)\end{array}$ & $\begin{array}{l}\text { TI } \\
\text { Conc. } \\
\left(\mu \mathrm{g} / \mathrm{m}^{3}\right)\end{array}$ & $\begin{array}{c}\mathrm{V} \\
\text { Conc. } \\
\left(\mu g / \mathrm{m}^{3}\right)\end{array}$ & $\begin{array}{c}Y \\
\text { Conc. } \\
\left(\mathrm{rg} / \mathrm{m}^{3}\right)\end{array}$ & $\begin{array}{c}\mathrm{Zn} \\
\text { Conc. } \\
\left(\mu g / \mathrm{m}^{3}\right)\end{array}$ & $\begin{array}{c}\text { Zr Conc. } \\
\left(\mu \mathrm{g} / \mathrm{m}^{3}\right)\end{array}$ \\
\hline SMTF-1A & 2.5 & $<0.16$ & $<0.12$ & 0.26 & 0.008 & 0.005 & 0.74 & 0.10 \\
\hline SMTF-1B & 1.0 & $<0.16$ & 0.1 & 0.02 & $<0.004$ & $<0.002$ & $<0.10$ & $<0.06$ \\
\hline SMTF-1C & 0.50 & $<0.16$ & 0.2 & 0.00 & $<0.004$ & 0.003 & $<0.10$ & $<0.06$ \\
\hline SMTF-1D & 0.25 & $<0.16$ & $<0.12$ & 0.00 & $<0.004$ & $<0.002$ & 0.14 & $<0.06$ \\
\hline SMTF-价 & $<0.25$ & $<0.16$ & $<0.12$ & $<0.002$ & $<0.004$ & $<0.002$ & 0.46 & $<0.06$ \\
\hline SMTF-1 & Total & $<0.16$ & 0.3 & 0.29 & $<0.004$ & 0.008 & 1.34 & 0.10 \\
\hline SMTF-2A & 2.5 & $<0.16$ & $<0.12$ & 0.12 & 0.006 & 0.005 & 0.47 & $<0.06$ \\
\hline SMTF-2B & 1.0 & $<0.16$ & $<0.12$ & 0.04 & $<0.004$ & $<0.002$ & $<0.10$ & $<0.06$ \\
\hline SMTF-2C & 0.50 & $<0.16$ & $<0.12$ & 0.01 & $<0.004$ & $<0.002$ & $<0.10$ & $<0.06$ \\
\hline SMTF-2D & 0.25 & $<0.16$ & $<0.12$ & 0.00 & $<0.004$ & $<0.002$ & $<0.10$ & $<0.06$ \\
\hline SMTF-2E & $<0.25$ & $<0.16$ & $<0.12$ & 0.01 & $<0.004$ & 0.002 & 0.23 & $<0.06$ \\
\hline SMTF-2 & Total & $<0.16$ & $<0.12$ & 0.18 & 0.006 & 0.007 & 0.70 & $<0.06$ \\
\hline SMTF-3A & 2.5 & $<0.27$ & $<0.20$ & 0.18 & $<0.007$ & $<0.003$ & 0.53 & $<0.10$ \\
\hline SMTF-3B & 1.0 & $<0.27$ & $<0.20$ & 0.01 & $<0.007$ & $<0.003$ & $<0.17$ & $<0.10$ \\
\hline SMTF-3C & 0.50 & $<0.27$ & $<0.20$ & $<0.003$ & $<0.007$ & $<0.003$ & $<0.17$ & $<0.10$ \\
\hline SMTF-3D & 0.25 & $<0.27$ & $<0.20$ & $<0.003$ & $<0.007$ & $<0.003$ & $<0.17$ & $<0.10$ \\
\hline SMTF-3E & $<0.25$ & $<0.27$ & $<0.20$ & $<0.003$ & $<0.007$ & $<0.003$ & $<0.17$ & $<0.10$ \\
\hline SMTF-3 & Total & $<0.27$ & $<0.20$ & 0.18 & $<0.007$ & $<0.003$ & 0.53 & $<0.10$ \\
\hline SMTF-4A & 2.5 & $<0.16$ & $<0.12$ & 0.10 & $<0.004$ & 0.016 & 0.42 & $<0.06$ \\
\hline SMTF-4B & 1.0 & $<0.16$ & $<0.12$ & 0,03 & $<0.004$ & 0.019 & 0.17 & $<0.06$ \\
\hline SMTF-4C & 0.50 & $<0.16$ & $<0.12$ & 0.01 & $<0.004$ & 0.048 & 0.12 & $<0.06$ \\
\hline SMTF-4D & 0.25 & $<0.16$ & $\leq 0.12$ & 0.00 & $<0.004$ & 0.170 & 0.42 & $<0.06$ \\
\hline SMTF-4E & $<0.25$ & $<0.16$ & $<0.12$ & 0.01 & 0.005 & 0.008 & 0.42 & $<0.06$ \\
\hline SMTF-4 & Total & $<0.16$ & $<0.12$ & 0.16 & 0.005 & 0.261 & 1.56 & $<0.06$ \\
\hline
\end{tabular}




\begin{tabular}{|c|c|c|c|c|c|c|c|c|}
\hline \multicolumn{9}{|c|}{$\begin{array}{l}\text { FACILITY \#1 - Copper/Beryllium Foundry and Machine Shop } \\
\text { Sioutas Cascade Impactor, Size-Selective Sample Results for Thirty-one Elements }\end{array}$} \\
\hline Sample Number & $\begin{array}{l}\text { Perticle } \\
\text { Slze } \\
(\mu \mathrm{m})\end{array}$ & $\begin{array}{c}\text { TI } \\
\text { Conc. } \\
\left(\mu g / \mathrm{m}^{3}\right)\end{array}$ & $\begin{array}{c}\text { Sn } \\
\text { Conc. } \\
\left(\mu g / \mathrm{m}^{3}\right)\end{array}$ & $\underset{\mathrm{Conc}}{\mathrm{TI}}$ & $\begin{array}{l}\text { Conc. } \\
\left(\mu g / \mathrm{m}^{3}\right)\end{array}$ & $\begin{array}{c}Y \\
\text { Conc. } \\
\left(\mu g / \mathrm{m}^{3}\right)\end{array}$ & $\begin{array}{c}\mathrm{Zn} \\
\text { Conc. } \\
\left(\mu \mathrm{g} / \mathrm{m}^{3}\right)\end{array}$ & $\begin{array}{c}\mathrm{Zr} \text { Conc. } \\
\left(\mu \mathrm{g} / \mathrm{m}^{3}\right)\end{array}$ \\
\hline SMTF-5A & 2.5 & $<0.16$ & $<0.12$ & 0.06 & $<0.004$ & $<0.002$ & 0.24 & $<0.06$ \\
\hline SMTF-5B & 1.0 & $<0.16$ & $<0.12$ & 0.01 & $<0.004$ & $<0.002$ & $<0.10$ & $<0.06$ \\
\hline SMTF-5C & 0.50 & $<0.16$ & $<0.12$ & 0.00 & $<0.004$ & $<0.002$ & $<0.10$ & $<0.06$ \\
\hline SMTF-5D & 0.25 & $<0.16$ & $<0.12$ & $<0.002$ & $<0.004$ & $<0.002$ & $<0.10$ & $<0.06$ \\
\hline SMTF-5E & $<0.25$ & $<0.16$ & $\leq 0.12$ & $<0.002$ & $<0.004$ & $<0.002$ & $<0.10$ & $<0.06$ \\
\hline SMTF-5 & Total & $<0.16$ & $<0.12$ & 0.07 & $<0.004$ & $<0.002$ & 0.24 & $<0.06$ \\
\hline SMTF-6A & 2.5 & $<0.17$ & $<0.13$ & 0.15 & 0.005 & 0.004 & 0.18 & $<0.06$ \\
\hline SMTF-6B & 1.0 & $<0.17$ & $<0.13$ & 0.06 & $<0.004$ & 0.002 & $<0.10$ & $<0.06$ \\
\hline SMTF-6C & 0.50 & $<0.17$ & $<0.13$ & 0.02 & $<0.004$ & $<0.002$ & $<0.10$ & $<0.06$ \\
\hline SMTF-6D & 0.25 & $<0.17$ & $<0.13$ & 0.01 & $<0.004$ & 0.004 & $<0.10$ & $<0.06$ \\
\hline SMTF-6E & $<0.25$ & $<0.17$ & 0.1 & 0.01 & 0.008 & 0.005 & $<0.10$ & $<0.06$ \\
\hline SMTF-6 & Total & $<0.17$ & 0.1 & 0.26 & 0.013 & 0.015 & 0.18 & $<0.06$ \\
\hline SMWF-1A & 2.5 & $<0.16$ & $<0.12$ & 0.04 & $<0.004$ & 0.008 & 0.13 & $<0.06$ \\
\hline SMWF-1B & 1.0 & $<0.16$ & $<0.12$ & 0.01 & $<0.004$ & $<0.002$ & $<0.10$ & $<0.06$ \\
\hline SMWF-1C & 0.50 & $<0.16$ & 0.1 & 0.01 & $<0.004$ & 0.002 & $<0.10$ & $<0.06$ \\
\hline SMWF-1D & 0.25 & $<0.16$ & 0.1 & 0.01 & $<0.004$ & 0.004 & $<0.10$ & $<0.06$ \\
\hline SMWF-1E & $<0.25$ & $<0.16$ & $<0.12$ & 0.03 & $<0.004$ & 0.004 & $<0.10$ & $<0.06$ \\
\hline SMWF-1 & Total & $<0.16$ & 0.3 & 0.10 & $<0.004$ & 0.018 & 0.13 & $<0.06$ \\
\hline SMWF-2A & 2.5 & $<0.17$ & $<0.12$ & 0.48 & 0.011 & 0.004 & 0.44 & $<0.06$ \\
\hline SMWF-2B & 1.0 & $<0.17$ & 0.1 & 0.19 & 0.005 & 0.002 & $<0.10$ & $<0.06$ \\
\hline SMWF-2C & 0.50 & $<0.17$ & $<0.12$ & 0.05 & $<0.004$ & 0.004 & $<0.10$ & $<0.06$ \\
\hline SMWF-2D & 0.25 & $<0.17$ & $<0.12$ & 0.02 & $<0.004$ & 0.003 & $<0.10$ & $<0.06$ \\
\hline SMWF-2E & $<0.25$ & $<0.17$ & $<0.12$ & 0.05 & 0.006 & 0.004 & $<0.10$ & $<0.06$ \\
\hline SMWF-2 & Total & $<0.17$ & 0.1 & 0.78 & 0.023 & 0.017 & 0.44 & $<0.06$ \\
\hline
\end{tabular}




\begin{tabular}{|c|c|c|c|c|c|c|c|c|}
\hline \multicolumn{9}{|c|}{$\begin{array}{l}\text { Appendix C - continued } \\
\text { FACILITY \#1 - Copper/Beryllium Foundry and Machine Shop } \\
\text { Sioutas Cascade Impactor, Size-Selective Sample Results for Thirty-one Elements }\end{array}$} \\
\hline Sample Number & $\begin{array}{c}\text { Partlcio } \\
\text { size } \\
(\mu \mathrm{m})\end{array}$ & $\begin{array}{c}\mathrm{Conc}_{\mathrm{H}_{3}} \\
\left(\mu \mathrm{g} / \mathrm{m}^{3}\right)\end{array}$ & $\begin{array}{c}\mathrm{Sn} \\
\text { Conci } \\
\left(\mu \mathrm{g} / \mathrm{m}^{3}\right)\end{array}$ & $\begin{array}{c}\mathrm{Tl} \\
\text { Conc, } \\
\left(\mathrm{\mu g} / \mathrm{m}^{3}\right)\end{array}$ & 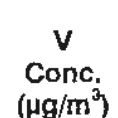 & 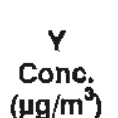 & $\begin{array}{c}\mathrm{Zn} \\
\mathrm{Conc} \\
\left(\mu \mathrm{g} / \mathrm{m}^{3}\right)\end{array}$ & $\begin{array}{c}\operatorname{Zr} \text { Conc. } \\
\left(\mu g / m^{3}\right)\end{array}$ \\
\hline SMWF-3A & 2.5 & $<0.17$ & $<0.12$ & 0.21 & 0.007 & $<0.002$ & 0.44 & $<0.06$ \\
\hline SMWF-3B & 1.0 & $<0.17$ & 0.1 & 0.04 & $<0.004$ & $<0.002$ & $<0.10$ & $<0.06$ \\
\hline SMWF-3C & 0.50 & $<0.17$ & $\leq 0.12$ & 0.01 & $<0.004$ & $<0.002$ & $<0.10$ & $<0.06$ \\
\hline SMWF-3D & 0.25 & $<0.17$ & $<0.12$ & 0.01 & $<0.004$ & $<0.002$ & $\overline{0.14}$ & $<0.06$ \\
\hline SMWF-3E & $<0.25$ & $<0.17$ & $<0.12$ & $<0.002$ & $<0.004$ & $<0.002$ & 0.46 & $<0.06$ \\
\hline SMWF-3 & Total & $<0.17$ & 0.1 & 0.27 & 0.007 & $<0.002$ & 1.03 & $<0.06$ \\
\hline
\end{tabular}

\begin{tabular}{|c|c|c|c|c|c|c|c|c|}
\hline SMWF-4A & 2.5 & $<0.16$ & $<0.12$ & 0.53 & 0.008 & 0.003 & 0.79 & $<0.06$ \\
\hline SMWF-4B & 1.0 & $<0.16$ & $<0.12$ & 0.17 & $<0.004$ & $<0.002$ & 0.13 & $<0.06$ \\
\hline SMWF-4C & 0.50 & $<0.16$ & $<0.12$ & 0.06 & $<0.004$ & $<0.002$ & $<0.10$ & $<0.06$ \\
\hline SMWF-4D & 0.25 & $<0.16$ & $<0.12$ & 0.01 & $<0.004$ & $<0.002$ & $<0.10$ & $<0.06$ \\
\hline SMWF-4E & $<0.26$ & $<0.16$ & $<0.12$ & 0.03 & $<0.004$ & 0.011 & $<0.10$ & $<0.06$ \\
\hline \hline SMWF-4 & Total & $<0.16$ & $<0.12$ & 0.81 & 0.008 & 0.014 & 0.93 & $<0.06$ \\
\hline
\end{tabular}

\begin{tabular}{|c|c|c|c|c|c|c|c|c|}
\hline SMWF-5A & 2.5 & $<0.17$ & $<0.13$ & 0.27 & $<0.004$ & 0.004 & 1.54 & 0.15 \\
\hline SMWF-5B & 1.0 & $<0.17$ & $<0.13$ & 0.07 & $<0.004$ & $<0.002$ & 0.35 & $<0.06$ \\
\hline SMWF-5C & 0.50 & $<0.17$ & $<0.13$ & 0.02 & $<0.004$ & $<0.002$ & $<0.10$ & $<0.06$ \\
\hline SMWF-5D & 0.25 & $<0.17$ & $<0.13$ & 0.01 & $<0.004$ & $<0.002$ & $<0.10$ & $<0.06$ \\
\hline SMWF-5E & $<0.25$ & $<0.17$ & $<0.13$ & 0.04 & $<0.004$ & 0.002 & $<0.10$ & $<0.06$ \\
\hline \hline SMWF-5 & Total & $<0.17$ & $<0.13$ & 0.42 & $<0.004$ & $<0.002$ & 1.90 & 0.15 \\
\hline
\end{tabular}

\begin{tabular}{|c|c|c|c|c|c|c|c|c|}
\hline SMWF-6A & 2.5 & $<0.16$ & $<0.12$ & 0.10 & $<0.004$ & 0.615 & 1.77 & $<0.06$ \\
\hline SMWF-6B & 1.0 & $<0.16$ & $<0.12$ & 0.03 & $<0.004$ & 0.519 & 1.36 & $<0.06$ \\
\hline SMWF-6C & 0.50 & $<0.16$ & $<0.12$ & 0.01 & $<0.004$ & 0.211 & 0.40 & $<0.06$ \\
\hline SMWF-60 & 0.25 & $<0.16$ & $<0.12$ & 0.00 & $<0.004$ & 0.171 & 0.48 & $<0.06$ \\
\hline SMWF-6E & $<0.25$ & $<0.16$ & $<0.12$ & 0.03 & $<0.004$ & 0.014 & 0.23 & $<0.06$ \\
\hline \hline SMWF-6 & Total & $<0.16$ & $<0.12$ & 0.16 & $<0.004$ & 1.530 & 4.25 & $<0.06$ \\
\hline
\end{tabular}




\begin{tabular}{|c|c|c|c|c|c|c|c|c|}
\hline \multicolumn{9}{|c|}{$\begin{array}{l}\text { FACILITY \#1 - Copper/Beryllium Foundry and Machine Shop } \\
\text { Sioutas Cascade Impactor, Size-Selective Sample Results for Thirty-one Elements }\end{array}$} \\
\hline Semple Number & $\begin{array}{l}\text { Particle } \\
\text { Size } \\
(\mu \mathrm{m})\end{array}$ & $\begin{array}{c}\text { T1 } \\
\text { Conc. } \\
\left(\mu g / \mathrm{m}^{3}\right)\end{array}$ & $\begin{array}{c}\text { Sn } \\
\text { Conc. } \\
\left(\mu \mathrm{g} / \mathrm{m}^{3}\right)\end{array}$ & $\begin{array}{c}\mathrm{Ti} \\
\text { Conc. } \\
\left(\mu \mathrm{g} / \mathrm{m}^{3}\right)\end{array}$ & $\begin{array}{c}v \\
\text { Conc. } \\
\left(\mu g / m^{3}\right)\end{array}$ & $\begin{array}{c}Y \\
\text { Conc. } \\
\left(\mu \mathrm{g} / \mathrm{m}^{3}\right)\end{array}$ & $\begin{array}{c}\mathrm{Zn} \\
\text { Conc. } \\
\left(\mu \mathrm{g} / \mathrm{m}^{3}\right)\end{array}$ & Zr Conc. $\left(\mu \mathrm{g} / \mathrm{m}^{3}\right)$ \\
\hline SMWF-7A & 2.5 & $<0.17$ & $<0.13$ & 0.09 & $<0.004$ & 0.019 & 0.30 & $<0.06$ \\
\hline SMWF-7B & 1.0 & $<0.17$ & $<0.13$ & 0.02 & $<0.004$ & 0.054 & 0.21 & $<0.06$ \\
\hline SMWF-7C & 0.50 & $<0.17$ & $<0.13$ & 0.00 & $<0.004$ & 0.039 & $<0.10$ & $<0.06$ \\
\hline SMWF-7D & 0.25 & $<0.17$ & $<0.13$ & $<0.002$ & $<0.004$ & 0.139 & 0.43 & $<0.06$ \\
\hline SMWF-7E & $<0.25$ & $<0.17$ & $<0.13$ & 0.03 & $<0.004$ & 0.003 & 0.19 & $<0.06$ \\
\hline SMWF-7 & Total & $<0.17$ & $<0.13$ & 0.14 & $\leq 0.004$ & 0.254 & 1.12 & $<0.06$ \\
\hline SMWF-BA & 2.5 & $<0.21$ & $<0.16$ & 0.01 & $<0.005$ & $<0.003$ & $<0.13$ & $<0.08$ \\
\hline SMWF-8B & 1.0 & $<0.21$ & $<0.16$ & $<0.003$ & $\leq 0.005$ & $<0.003$ & $<0.13$ & $<0.08$ \\
\hline SMWF-8C & $\underline{0.50}$ & $<0.21$ & $<0.16$ & $<0.003$ & $<0.005$ & $<0,003$ & $<0.13$ & $<0.08$ \\
\hline SMWF-8D & 0.25 & $<0.21$ & $<0.16$ & $<0.003$ & $<0.005$ & $<0.003$ & $<0.13$ & $<0.08$ \\
\hline SMWF-8E & $<0.25$ & $<0.21$ & $<0.16$ & 0.03 & $<0.005$ & 0.007 & $<0.13$ & $<0.08$ \\
\hline SMWF-8 & Total & $<0.21$ & $<0.16$ & 0.04 & $<0.005$ & 0.007 & $<0.13$ & $<0.08$ \\
\hline
\end{tabular}




\begin{tabular}{|c|c|c|c|c|c|c|c|c|c|c|c|c|c|c|}
\hline \multicolumn{15}{|c|}{$\begin{array}{l}\text { FACILITY \#1 - Copper/Beryllium Foundry and Machine Shop } \\
\text { MOUDI Impactor Size-Selective Air Sample Results for Total Particulate and Thirty-one Elements }\end{array}$} \\
\hline $\begin{array}{l}\text { Sample } \\
\text { Number }\end{array}$ & $\begin{array}{l}\text { Particle } \\
\text { Size } \\
(\mu \mathrm{m})\end{array}$ & $\begin{array}{l}\text { Particulate } \\
\text { Conc. } \\
\left(\mathbf{m g} / \mathrm{m}^{3}\right)\end{array}$ & $\begin{array}{c}\text { Al } \\
\text { Conc. } \\
\left(\mu \mathrm{g} / \mathrm{m}^{3}\right)\end{array}$ & $\begin{array}{c}\text { Sb } \\
\text { Conc. } \\
\left(\mu g / m^{3}\right)\end{array}$ & $\begin{array}{c}\text { As } \\
\text { Conc. } \\
\left(\mu \mathrm{g} / \mathrm{m}^{3}\right)\end{array}$ & $\begin{array}{l}\text { Ba } \\
\text { Conc. } \\
\left(\mu g / m^{3}\right)\end{array}$ & $\begin{array}{c}\text { Be } \\
\text { Conc. } \\
\left(\mu g / m^{3}\right)\end{array}$ & $\begin{array}{c}\text { Cd } \\
\text { Conc. } \\
\left(\mu g / m^{3}\right)\end{array}$ & $\begin{array}{c}\text { Ca } \\
\text { Conc. } \\
\left(\mu g / m^{3}\right)\end{array}$ & $\begin{array}{c}\mathrm{Cr} \\
\text { Conc. } \\
\left(\mu g / m^{3}\right)\end{array}$ & $\begin{array}{c}\text { Co } \\
\text { Conc. } \\
\left(\mu g / m^{3}\right)\end{array}$ & $\begin{array}{c}\text { Cu } \\
\text { Conc. } \\
\left(\mu \mathrm{g} / \mathrm{m}^{3}\right)\end{array}$ & $\begin{array}{c}F \theta \\
\text { Conc. } \\
\left(\mu g / m^{3}\right)\end{array}$ & $\begin{array}{c}\mathrm{La} \\
\text { Conc. } \\
\left(\mu \mathrm{g} / \mathrm{m}^{3}\right)\end{array}$ \\
\hline $\mathrm{A} 1-0$ & $>18$ & 0.11 & 0.49 & $<0.02$ & 0.05 & $<0.002$ & 0.033 & $<0.001$ & $<0.70$ & 0.008 & 0.013 & 0.77 & 0.22 & 0.001 \\
\hline $\mathrm{A} 1-1$ & 10 & 0.15 & 0.40 & $<0.02$ & $<0.04$ & $<0.002$ & 0.014 & $<0.001$ & $<0.70$ & $<0.004$ & 0.008 & 0.47 & $<0.14$ & $<0.001$ \\
\hline $\mathrm{A} 1-2$ & 5.6 & 0.10 & 0.56 & $<0.02$ & 0.05 & $<0.002$ & 0.030 & $<0.001$ & 0.84 & 0.007 & 0.010 & 0.63 & 0.48 & $<0.001$ \\
\hline $\mathrm{A} 1-3$ & 3.2 & 0.07 & 0.20 & $<0.02$ & 0.07 & $<0.002$ & 0.007 & $<0.001$ & $<0.7 \overline{0}$ & $<0.004$ & $<0.006$ & 0.22 & $<0.14$ & $<0.001$ \\
\hline$A 1-4$ & 1.8 & 0.19 & 0.41 & $<0.02$ & $<0.04$ & 0.003 & 0.023 & $<0.001$ & 2.16 & 0.006 & $<0.006$ & 0.38 & 0.16 & $<0.001$ \\
\hline$A 1-5$ & 1.0 & 0.11 & 0.28 & $<0.02$ & $<0.04$ & $<0.002$ & 0.005 & $<0.001$ & $<0.70$ & 0.010 & $<0.006$ & 0.13 & $<0.14$ & $<0.001$ \\
\hline $\mathrm{A} 1-6$ & 0.56 & 0.12 & 0.37 & $<0.02$ & $<0.04$ & $<0.002$ & $<0.003$ & 0.002 & $<0.70$ & 0.012 & $<0.006$ & 0.03 & $<0.14$ & $<0.001$ \\
\hline A1-7 & $\overline{0.32}$ & 0.05 & 0.24 & $<0.02$ & $<0.04$ & 0.019 & $<0.003$ & $<0.001$ & 1.53 & 0.008 & $<0.006$ & 0.02 & $<0.14$ & $<0.001$ \\
\hline$A 1-8$ & 0.18 & 0.06 & 0.18 & $<0.02$ & $<0.04$ & $<0.002$ & $<0.003$ & 0.002 & 1.25 & 0.006 & $<0.006$ & 0.02 & $<0.14$ & $<0.001$ \\
\hline A1-9 & 0.1 & 0.13 & 0.11 & $<0.02$ & $<0.04$ & $<0.002$ & $<0.003$ & $<0.001$ & $<0.70$ & 0.013 & $<0.006$ & 0.02 & $<0.14$ & $<0.001$ \\
\hline A1-10 & 0.056 & 0.08 & 0.10 & $<0.02$ & $<0.04$ & $<0.002$ & $<0.003$ & $<0.001$ & $<0.70$ & 0.008 & $<0.006$ & 0.01 & $<0.14$ & $<0.001$ \\
\hline $\mathrm{A} 1-\mathrm{F}$ & $<0.056$ & 0.01 & 0.17 & $<0.02$ & $<0.04$ & $<0.002$ & $<0.003$ & $<0.001$ & $<0.70$ & $<0.004$ & $<0.006$ & 0.11 & $<0.14$ & $<0.001$ \\
\hline A1-total & & 1.18 & 3.50 & $<0.02$ & 0.17 & 0.023 & 0.111 & 0.004 & 5.78 & 0.077 & 0.031 & 2.82 & 0.86 & 0.001 \\
\hline B1-0 & $>18$ & 0.04 & 0.69 & $<0.02$ & $<0.04$ & $<0.002$ & 0.070 & $<0.001$ & 1,34 & $<0.004$ & 0.066 & 1.42 & 0.45 & $<0.001$ \\
\hline B1-1 & 10 & 0.02 & 0.21 & $<0.02$ & $<0.04$ & $<0.002$ & 0.022 & $<0.001$ & 1.34 & $<0.004$ & 0.028 & 0.57 & $<0.15$ & $<0.001$ \\
\hline B1-2 & 5.6 & 0.03 & 0.31 & $<0.02$ & 0.05 & 0.003 & 0.041 & $<0.001$ & 2.31 & $<0.004$ & 0.043 & 0.75 & 0.31 & $<0.001$ \\
\hline B1-3 & 3.2 & 0.04 & 0.43 & $<0.02$ & 0.05 & 0.004 & 0.053 & $<0.001$ & 2.68 & $<0.004$ & 0.063 & 1.04 & 0.60 & $<0.001$ \\
\hline B1-4 & 1.8 & 0.01 & 0.13 & $<0.02$ & $<0.04$ & $<0.002$ & 0.010 & 0.010 & $<0.75$ & $<0.004$ & $<0.007$ & 0.16 & $<0.15$ & $<0.001$ \\
\hline By -5 & 1.0 & 0.02 & 0.21 & $<0.02$ & $<0.04$ & $<0.002$ & 0.019 & $<0.001$ & 0.89 & $<0.004$ & 0.015 & 0.40 & $<0.15$ & $<0.001$ \\
\hline B1-6 & 0.56 & 0.08 & 0.18 & $<0.02$ & $<0.04$ & $<0.002$ & 0.006 & $<0.001$ & $<0.75$ & $<0.004$ & $<0.007$ & 0.10 & $<0.15$ & $<0.001$ \\
\hline B1-7 & 0.32 & 0.01 & 0.11 & $<0.02$ & $<0.04$ & $<0.002$ & 0.008 & $<0.001$ & $<0.75$ & $<0.004$ & $<0.007$ & 0.07 & $<0.15$ & $<0.001$ \\
\hline B1-8 & 0.18 & 0.01 & $<0.04$ & $<0.02$ & $<0.04$ & $<0.002$ & 0.007 & 0.002 & $<0.75$ & $<0.004$ & $<0.007$ & 0.10 & $<0.15$ & $<0.001$ \\
\hline B1-9 & 0.1 & 0.07 & $<0.04$ & $<0.02$ & $<0.04$ & $<0.002$ & $<0.004$ & $<0.001$ & $<0.75$ & 0.005 & $<0.007$ & 0.11 & $<0.15$ & $<0.001$ \\
\hline B1-10 & 0.056 & 0.03 & $<0.04$ & $<0.02$ & $<0.04$ & $<0.002$ & $<0.004$ & $<0.001$ & $<0.75$ & $<0.004$ & $<0.007$ & 0.01 & $<0.15$ & $<0.001$ \\
\hline $\mathrm{B} 1-\mathrm{F}$ & $<0.056$ & 0.02 & $<0.04$ & $<0.02$ & $<0.04$ & $<0.002$ & $<0.004$ & $<0.001$ & $<0.75$ & $<0.004$ & $<0.007$ & 0.02 & $<0.15$ & $<0.001$ \\
\hline B1-total & & 0.36 & 2.26 & $<0.02$ & 0.10 & 0.007 & 0.237 & 0.011 & 8.58 & 0.005 & 0.216 & 4.76 & 1.36 & $<0.001$ \\
\hline
\end{tabular}




\section{Appendix D - continued}

FACILITY \#1 - Copper/Beryllium Foundry and Machine Shop MOUDI Impactor Size-Selective Air Sample Results for Total Particulate and Thirty-one Elements

\begin{tabular}{|c|c|c|c|c|c|c|c|c|c|c|c|c|c|c|}
\hline $\begin{array}{l}\text { Sample } \\
\text { Number }\end{array}$ & $\begin{array}{c}\text { Particle } \\
\text { Size } \\
(\mu \mathrm{m})\end{array}$ & $\begin{array}{c}\text { Particulate } \\
\text { Conc. } \\
\left(\mathrm{mg} / \mathrm{m}^{3}\right)\end{array}$ & $\begin{array}{c}\text { Al } \\
\text { Conc. } \\
\left(\mu \mathrm{g} / \mathrm{m}^{3}\right)\end{array}$ & $\begin{array}{c}\text { Sb } \\
\text { Conc. } \\
\left(\mu \mathrm{g} / \mathrm{m}^{3}\right)\end{array}$ & $\begin{array}{c}\text { As } \\
\text { Conc. } \\
\left(\mu \mathrm{g} / \mathrm{m}^{3}\right)\end{array}$ & $\begin{array}{c}\text { Ba } \\
\text { Conc. } \\
\left(\mu g / \mathrm{m}^{3}\right)\end{array}$ & $\begin{array}{c}\mathrm{Be} \\
\mathrm{Conc} \\
\left(\mu \mathrm{g} / \mathrm{m}^{3}\right)\end{array}$ & $\begin{array}{c}\text { Cd } \\
\text { Conc. } \\
\left(\mu \mathrm{g} / \mathrm{m}^{3}\right)\end{array}$ & $\begin{array}{c}\text { Ca } \\
\text { Conc. } \\
\left(\mu g / m^{3}\right)\end{array}$ & $\begin{array}{c}\mathrm{Cr} \\
\text { Conc. } \\
\left(\mu \mathrm{g} / \mathrm{m}^{3}\right)\end{array}$ & $\begin{array}{c}\text { Co } \\
\text { Conc. } \\
\left(\mu g / \mathrm{m}^{3}\right)\end{array}$ & $\begin{array}{c}\text { Cu } \\
\text { Conc. } \\
\left(\mu g / m^{3}\right)\end{array}$ & $\begin{array}{c}\text { Fe } \\
\text { Conc. } \\
\left(\mu g / m^{3}\right)\end{array}$ & $\begin{array}{c}\text { La } \\
\text { Conc. } \\
\left.\text { ( } \mu \mathrm{g} / \mathrm{m}^{3}\right)\end{array}$ \\
\hline C1-0 & $>18$ & 0.09 & 0.61 & $<0.02$ & $<0.05$ & 0.003 & 0.126 & $<0.002$ & 1.65 & 0.008 & 0.102 & 10.22 & 3.14 & $<0.001$ \\
\hline C1-1 & 10 & 0.07 & 0.79 & $<0.02$ & $<0.05$ & 0.004 & 0.079 & $<0.002$ & 2.12 & 0.009 & 0.069 & 6.60 & 2.91 & $<0.001$ \\
\hline C1-2 & 5.6 & 0.06 & 0.48 & $<0.02$ & $<0.05$ & $<0.002$ & 0.037 & $<0.002$ & 2.20 & $<0.005$ & 0.031 & 2.75 & 1.81 & $<0.001$ \\
\hline C1-3 & 3.2 & 0.04 & 0.72 & $<0.02$ & $<0.05$ & 0.004 & 0.039 & $<0.002$ & 2.59 & $<0.005$ & 0.035 & 3.07 & 2.20 & $<0.001$ \\
\hline C1-4 & 1.8 & 0.02 & 0.31 & $<0.02$ & $<0.05$ & $<0.002$ & 0.017 & $<0.002$ & 1.10 & $<0.005$ & 0.015 & 1.42 & 0.78 & $<0.001$ \\
\hline $\mathrm{C} 1-5$ & 1.0 & 0.02 & 0.18 & $<0.02$ & $<0.05$ & 0.003 & 0.009 & $<0.002$ & 3.22 & $<0.005$ & $<0.007$ & 0.61 & 0.40 & $<0.001$ \\
\hline C1-6 & 0.56 & 0.01 & $<0.05$ & $<0.02$ & $<0.05$ & $<0.002$ & $<0.004$ & $<0.002$ & $<0.79$ & $<0.005$ & $<0.007$ & 0.07 & $\leq 0.16$ & $<0.001$ \\
\hline C1-7 & 0.32 & 0.01 & $<0.05$ & $<0.02$ & $<0.05$ & $<0.002$ & $<0.004$ & $<0.002$ & $<0.79$ & $<0.005$ & $<0.007$ & 0.04 & $<0.16$ & $<0.001$ \\
\hline C1-8 & 0.18 & 0.00 & $<0.05$ & $<0.02$ & $<0.05$ & $<0.002$ & $<0.004$ & $<0.002$ & 0.86 & $<0.005$ & $<0.007$ & 0.03 & $<0.16$ & $<0.001$ \\
\hline C1-9 & 0.1 & 0.01 & $<0.05$ & $<0.02$ & $<0.05$ & $<0.002$ & $<0.004$ & $<0.002$ & $<0.79$ & $<0.005$ & $<0.007$ & $<0.005$ & $<0.16$ & $<0.001$ \\
\hline$C 1-10$ & 0.056 & 0.00 & $<0.05$ & $<0.02$ & $<0.05$ & $<0.002$ & $<0.004$ & $<0.002$ & $<0.79$ & $<0.005$ & $<0.007$ & $<0.005$ & $<0.16$ & $<0.001$ \\
\hline $\mathrm{C} 1-\mathrm{F}$ & $<0.056$ & 0.03 & $<0.05$ & $<0.02$ & $<0.05$ & $<0.002$ & $<0.004$ & $<0.002$ & $<0.79$ & $<0.005$ & $<0.007$ & $<0.005$ & $<0.16$ & $<0.001$ \\
\hline C1-total & & 0.35 & 3.10 & $<0.02$ & $<0.05$ & 0.014 & 0.306 & $<0.002$ & 13.76 & 0.016 & 0.252 & 24.80 & 11.24 & $<0.001$ \\
\hline & & & & & & & & & & & & & & \\
\hline A2-0 & $>18$ & 0.05 & 0.45 & $<0.02$ & $<0.04$ & $<0.002$ & 0.031 & $<0.001$ & 0.90 & $<0.004$ & 0.014 & 0.90 & 0.19 & $<0.001$ \\
\hline A2-1 & 10 & 0.02 & 0.37 & $<0.02$ & $<0.04$ & $<0.002$ & 0.023 & $<0.001$ & $<0.70$ & $<0.004$ & 0.010 & 0.68 & $<0.14$ & $<0.001$ \\
\hline A2-2 & 5.6 & 0.03 & 0.37 & $<0.02$ & $<0.04$ & 0.002 & 0.018 & $<0.001$ & 2.00 & $<0.004$ & 0.010 & 0.61 & 0.14 & $<0.001$ \\
\hline A2-3 & 3.2 & 0.02 & 0.25 & $<0.02$ & $<0.04$ & $<0.002$ & 0.019 & $<0.001$ & 0.76 & $<0.004$ & 0.007 & 0.52 & $<0.14$ & $<0.001$ \\
\hline A2-4 & 1.8 & 0.02 & 0.12 & $<0.02$ & $<0.04$ & 0.002 & 0.014 & $<0.001$ & $<0.69$ & $<0.004$ & $<0.006$ & 0.30 & $<0.14$ & $<0.001$ \\
\hline A2-5 & 1.0 & 0.02 & 0.05 & $<0.02$ & $<0.04$ & $<0.002$ & $<0.003$ & $<0.001$ & $<0.69$ & $<0.004$ & $<0.006$ & 0.12 & $<0 . \overline{14}$ & $<0.001$ \\
\hline A2-6 & 0.56 & 0.03 & 0.13 & $<0.02$ & $<0.04$ & $<0.002$ & $<0.003$ & $<0.001$ & 1.04 & $<0.004$ & $<0.006$ & 0.07 & $<0.14$ & $<0.001$ \\
\hline A2-7 & 0.32 & 0.02 & 0.09 & $<0.02$ & $<0.04$ & $<0.002$ & $<0.003$ & $<0.001$ & $<0.69$ & $<0.004$ & $<0.006$ & 0.04 & $<0.14$ & $<0.001$ \\
\hline A2-8 & 0.18 & 0.02 & $<0.04$ & $<0.02$ & $<0.04$ & $<0.002$ & $<0.003$ & $<0.001$ & 0.83 & $<0.004$ & $<0.006$ & 0.05 & $<0.14$ & $<0.001$ \\
\hline A2-9 & 0.1 & 0.01 & $<0.04$ & $<0.02$ & $<0.04$ & $<0.002$ & $<0.003$ & 0.002 & 1.24 & $<0.004$ & $<0.006$ & 0.07 & $<0.14$ & $<0.001$ \\
\hline A2-10 & 0.056 & 0.02 & $<0.04$ & $<0.02$ & $<0.04$ & $<0.002$ & $<0.003$ & $<0.001$ & $<0.69$ & $<0.004$ & $<0.006$ & 0.01 & $<0.14$ & $<0.001$ \\
\hline A2- $F$ & $<0.056$ & 0.01 & 0.08 & $<0.02$ & $<0.04$ & $<0.002$ & $<0.003$ & 0.003 & $<0.69$ & 0.013 & $<0.006$ & 0.08 & $<0.14$ & $<0.001$ \\
\hline A2-total & & 0.27 & 1.89 & $<0.02$ & $<0.04$ & 0.004 & 0.106 & 0.005 & 6.76 & 0.013 & 0.041 & 3.44 & 0.33 & $<0.001$ \\
\hline
\end{tabular}




\section{Appendix D - continued}

FACILITY \#1 - Copper/Beryllium Foundry and Machine Shop MOUDI Impactor Size-Selective Air Sample Results for Total Particulate and Thirty-one Elements

\begin{tabular}{|c|c|c|c|c|c|c|c|c|c|c|c|c|c|c|}
\hline $\begin{array}{l}\text { Sample } \\
\text { Number }\end{array}$ & $\begin{array}{c}\text { Particle } \\
\text { Size } \\
(\mu \mathrm{m})\end{array}$ & $\begin{array}{c}\text { Particulate } \\
\text { Conc. } \\
\left.\text { (mg/m } / \mathrm{m}^{3}\right)\end{array}$ & $\begin{array}{c}\text { Al } \\
\text { Conc. } \\
\left(\mu \mathrm{g} / \mathrm{m}^{3}\right)\end{array}$ & $\begin{array}{c}\text { Sb } \\
\text { Conc. } \\
\left(\mu g / m^{3}\right)\end{array}$ & $\begin{array}{c}\text { As } \\
\text { Conc. } \\
\left(\mu \mathrm{g} / \mathrm{m}^{3}\right)\end{array}$ & $\begin{array}{c}\text { Ba } \\
\text { Conc. } \\
\left(\mu g / m^{3}\right)\end{array}$ & $\begin{array}{c}\text { Be } \\
\text { Conc. } \\
\left(\mu g / m^{3}\right)\end{array}$ & $\begin{array}{c}\text { Cd } \\
\text { Conc. } \\
\left(\mu g / m^{3}\right)\end{array}$ & $\begin{array}{c}\text { Ca } \\
\text { Conc. } \\
\left(\mu g / m^{3}\right)\end{array}$ & $\begin{array}{c}\mathrm{Cr} \\
\text { Conc. } \\
\left(\mu \mathrm{g} / \mathrm{m}^{3}\right)\end{array}$ & $\begin{array}{c}\text { Co } \\
\text { Conc. } \\
\left(\mu \mathrm{g} / \mathrm{m}^{3}\right)\end{array}$ & $\begin{array}{c}\mathrm{Cu} \\
\text { Conc. } \\
\left(\mu \mathrm{g} / \mathrm{m}^{3}\right)\end{array}$ & $\begin{array}{c}F e \\
\text { Conc. } \\
\left(\mu g / m^{3}\right)\end{array}$ & $\begin{array}{c}\text { La } \\
\text { Conc. } \\
\left(\mu \mathrm{g} / \mathrm{m}^{3}\right)\end{array}$ \\
\hline B2-0 & $>18$ & na & 0.13 & $<0.02$ & $<0.04$ & $<0.002$ & 0.026 & $<0.001$ & 1.05 & $<0.004$ & 0.023 & 0.61 & $<0.14$ & $<0.001$ \\
\hline B2-1 & 10 & na & $0 . \overline{19}$ & $<0.02$ & $<0.04$ & $<0.002$ & 0.032 & $<0.001$ & 0.98 & $<0.004$ & 0.039 & 0.84 & $<0.14$ & $<0.001$ \\
\hline B2-2 & 5.6 & na & 0.14 & $<0.02$ & $<0.04$ & $<0.002$ & 0.020 & $<0.001$ & 0.70 & $<0.004$ & 0.038 & 0.56 & $<0.14$ & $<0.001$ \\
\hline B2-3 & 3.2 & na & $<0.04$ & $<0.02$ & $<0.04$ & $<0.002$ & 0.012 & $<0.001$ & $<0.70$ & $<0.004$ & 0.019 & 0.36 & $<0.14$ & $<0.001$ \\
\hline B2-4 & 1.8 & na & $<0.04$ & $<0.02$ & $<0.04$ & $<0.002$ & 0.004 & $<0.001$ & 0.77 & $<0.004$ & $<0.006$ & 0.10 & $<0.14$ & $<0.001$ \\
\hline B2-5 & 1.0 & na & $<0.04$ & $<0.02$ & $<0.04$ & $<0.002$ & 0.004 & $<0.001$ & $<0.70$ & $<0.004$ & $<0.006$ & 0.11 & $<0.14$ & $<0.001$ \\
\hline B2-6 & 0.56 & na & 0.06 & $<0.02$ & $<0.04$ & $<0.002$ & 0.005 & $<0.001$ & 1.62 & $<0.004$ & $<0.006$ & 0.08 & $<0.14$ & $<0.001$ \\
\hline B2-7 & 0.32 & na & 0.05 & $<0.02$ & $<0.04$ & $<0.002$ & 0.006 & $<0.001$ & 0.98 & $<0.004$ & $<0.006$ & 0.09 & $<0.14$ & $<0.001$ \\
\hline B2-8 & 0.18 & na & $<0.04$ & $<0.02$ & $<0.04$ & $<0.002$ & 0.005 & $<0.001$ & $<0.70$ & $<0.004$ & $<0.006$ & 0.12 & $<0.14$ & $<0.001$ \\
\hline B2-9 & 0.1 & na & $<0.04$ & $<0.02$ & $<0.04$ & $<0.002$ & $<0.004$ & $<0.001$ & 0.77 & $<0.004$ & $<0.006$ & 0.06 & $<0.14$ & $<0.001$ \\
\hline B2-10 & 0.056 & na & $<0.04$ & $<0.02$ & $<0.04$ & $<0.002$ & $<0.004$ & $<0.001$ & $<0.70$ & $<0.004$ & $<0.006$ & 0.01 & $<0.14$ & $<0.001$ \\
\hline B2-F & $<0.056$ & na & $<0.04$ & $<0.02$ & $<0.04$ & $<0.002$ & $<0.004$ & 0.001 & $<0.70$ & $<0.004$ & $<0.006$ & 0.01 & $<0.14$ & $<0.001$ \\
\hline B2-total & & na & 0.57 & $<0.02$ & $<0.04$ & $<0.002$ & 0.116 & 0.001 & 6.89 & $<0.004$ & 0.120 & 2.95 & $<0.14$ & $<0.001$ \\
\hline & & & & & & & & & & & & & & \\
\hline $\mathrm{C} 2-0$ & $>18$ & 0.05 & 0.52 & $<0.02$ & $<0.04$ & 0.003 & 0.331 & $<0.001$ & 1.08 & $<0.004$ & 0.070 & 12.96 & 1.37 & $<0.001$ \\
\hline C2-1 & 10 & 0.04 & 0.24 & $<0.02$ & $<0.04$ & $<0.002$ & 0.187 & $<0.001$ & $<0.72$ & $<0.004$ & 0.037 & 7.13 & 0.72 & $<0.001$ \\
\hline $\mathrm{C} 2-2$ & 5.6 & 0.05 & 0.59 & $<0.02$ & $<0.04$ & 0.003 & 0.166 & $<0.001$ & 1.30 & $<0.004$ & 0.033 & 5.90 & 1.58 & $<0.001$ \\
\hline $\mathrm{C} 2-3$ & 3.2 & 0.03 & 0.53 & $<0.02$ & $<0.04$ & 0.002 & 0.094 & $<0.001$ & 1.44 & $<0.004$ & 0.019 & 3.24 & 0.86 & $<0.001$ \\
\hline C2-4 & 1.8 & 0.02 & 0.23 & $<0.02$ & $<0.04$ & $<0.002$ & 0.029 & $<0.001$ & 0.94 & $<0.004$ & $<0.006$ & 1.01 & 0.86 & $<0.001$ \\
\hline $\mathrm{C} 2-5$ & 1.0 & 0.02 & 0.29 & $<0.02$ & $<0.04$ & $<0.002$ & 0.045 & $<0.001$ & 0.72 & $<0.004$ & 0.009 & 1.44 & 1.01 & $<0.001$ \\
\hline $\mathrm{C} 2-6$ & 0.56 & 0.01 & 0.08 & $<0.02$ & $<0.04$ & $<0.002$ & 0.012 & $<0.001$ & $<0.72$ & $<0.004$ & $<0.006$ & 0.37 & 0.19 & $<0.001$ \\
\hline C2-7 & 0.32 & 0.01 & $<0.04$ & $<0.02$ & $<0.04$ & $<0.002$ & 0.004 & $<0.001$ & $<0.72$ & $<0.004$ & $<0.006$ & 0.11 & 0.33 & $<0.001$ \\
\hline C2-8 & 0.18 & 0.00 & $<0.04$ & $<0.02$ & $<0.04$ & $<0.002$ & $<0.004$ & $<0.001$ & $<0.72$ & $<0.004$ & $<0.006$ & 0.07 & 0.15 & $<0.001$ \\
\hline C2-9 & 0.1 & 0.00 & $<0.04$ & $<0.02$ & $<0.04$ & $<0.002$ & $<0.004$ & $<0.001$ & $<0.72$ & $<0.004$ & $<0.006$ & 0.05 & 0.50 & $<0.001$ \\
\hline $\mathrm{C} 2-10$ & 0.056 & 0.01 & $<0.04$ & $<0.02$ & $<0.04$ & $<0.002$ & $<0.004$ & $<0.001$ & $<0.72$ & $<0.004$ & $<0.006$ & 0.01 & $<0.14$ & $<0.001$ \\
\hline $\mathrm{C} 2-\mathrm{F}$ & $<0.056$ & 0.02 & $<0.04$ & $<0.02$ & $<0.04$ & $<0.002$ & $<0.004$ & $<0.001$ & $<0.72$ & $<0.004$ & $<0.006$ & 0.04 & $<0.14$ & $<0.001$ \\
\hline C2-total & & 0.27 & 2.48 & $<0.02$ & $<0.04$ & 0.008 & 0.867 & $<0.001$ & 5.47 & $<0.004$ & 0.169 & 32.32 & 7.58 & $<0.001$ \\
\hline
\end{tabular}




\begin{tabular}{|c|c|c|c|c|c|c|c|c|c|c|c|c|}
\hline \multicolumn{13}{|c|}{$\begin{array}{l}\text { FACILITY \#1 - Copper/Beryllium Foundry and Machine Shop } \\
\text { MOUDI Impactor Size-Selective Air Sample Results for Total Particulate and Thirty-one Elements }\end{array}$} \\
\hline $\begin{array}{l}\text { Sample } \\
\text { Number }\end{array}$ & $\begin{array}{l}\text { Particle } \\
\text { Size } \\
(\mu \mathrm{m})\end{array}$ & $\begin{array}{c}\mathrm{Pb} \\
\text { Conc. } \\
\left(\mu \mathrm{g} / \mathrm{m}^{3}\right)\end{array}$ & $\begin{array}{c}\mathrm{Li} \\
\text { Conc. } \\
\left(\mu g / \mathrm{m}^{3}\right)\end{array}$ & $\begin{array}{c}M g \\
\text { Conc. } \\
\left(\mu g / m^{3}\right)\end{array}$ & $\begin{array}{c}\text { Mn } \\
\text { Conc. } \\
\left(\mu g / m^{3}\right)\end{array}$ & $\begin{array}{l}\text { Mo } \\
\text { Conc. } \\
\left(\mu g / \mathrm{m}^{3}\right)\end{array}$ & $\begin{array}{c}\mathrm{Ni} \\
\text { Conc. } \\
\left(\mu \mathrm{g} / \mathrm{m}^{3}\right)\end{array}$ & $\begin{array}{c}P \\
\text { Conc, } \\
\left\langle\mu g / m^{3}\right)\end{array}$ & $\begin{array}{c}K \\
\text { Conc. } \\
\left(\mu \mathrm{g} / \mathrm{m}^{3}\right)\end{array}$ & $\begin{array}{c}\text { Se } \\
\text { Conc. } \\
\left(\mu g / m^{3}\right)\end{array}$ & $\begin{array}{c}\mathrm{Ag} \\
\text { Conc. } \\
\left(\mu \mathrm{g} / \mathrm{m}^{3}\right)\end{array}$ & $\begin{array}{c}\mathrm{Sr} \\
\text { Conc. } \\
\left(\mu \mathrm{g} / \mathrm{m}^{3}\right)\end{array}$ \\
\hline $\mathrm{A} 1-0$ & $>18$ & $<0.014$ & $<0.005$ & 0.37 & $<0.006$ & $<0.007$ & $\leq 0.03$ & $<0.63$ & $<0.07$ & $<0.14$ & $<0.001$ & $<0.004$ \\
\hline$\overline{A 1-1}$ & 10 & $<0.014$ & $<0.005$ & 0.30 & $<0.006$ & $<0.007$ & $\leq 0.03$ & $<0.63$ & $<0.07$ & $<0.14$ & $<0.001$ & $<0.004$ \\
\hline$A 1-2$ & 5.6 & $<0.014$ & $<0.005$ & 0.46 & $<0.006$ & $<0.007$ & $<0.03$ & $<0.63$ & $<0.07$ & $<0.14$ & $<0.001$ & $<0.004$ \\
\hline A1-3 & 3.2 & $<0.014$ & $<0.005$ & $<0.21$ & $<0.006$ & $<0.007$ & $<0.03$ & $<0.63$ & $<0.07$ & $<0.14$ & $<0.001$ & $<0.004$ \\
\hline$A 1-4$ & 1.8 & $<0.014$ & $<0.005$ & 0.63 & $<0.006$ & $<0.007$ & $<0.03$ & 1.18 & $<0.07$ & $<0.14$ & $<0.001$ & 0.008 \\
\hline$A 1-5$ & 1.0 & $<0.014$ & $<0.005$ & $<0.21$ & $<0.006$ & $<0.007$ & $<0.03$ & $<0.63$ & $<0.07$ & $<0.14$ & $<0.001$ & $<0.004$ \\
\hline A1-6 & 0.56 & $<0.014$ & $<0.005$ & $<0.21$ & $<0.006$ & $<0.007$ & $<0.03$ & $<0.63$ & $<0.07$ & $<0.14$ & $<0.001$ & $<0.004$ \\
\hline $\mathrm{A} 1-7$ & 0.32 & $<0.014$ & $<0.005$ & 0.45 & $<0.006$ & $<0.007$ & $<0.03$ & 0.97 & $<0.07$ & $<0.14$ & $<0.001$ & 0.007 \\
\hline$A 1-8$ & 0.18 & $<0.014$ & $<0.005$ & 0.31 & $<0.006$ & $<0.007$ & $<0.03$ & 0.97 & $<0.07$ & $<0.14$ & $<0.001$ & 0.006 \\
\hline A1-9 & 0.1 & $<0.014$ & $<0.005$ & $<0.21$ & $<0.006$ & $<0.007$ & $<0.03$ & $<0.63$ & $<0.07$ & $<0.14$ & $<0.001$ & $<0.004$ \\
\hline$A 1-10$ & 0.056 & $<0.014$ & $<0.005$ & $<0.21$ & $<0.006$ & $<0.007$ & $<0.03$ & $<0.63$ & $<0.07$ & $<0.14$ & $<0.001$ & $<0.004$ \\
\hline A1-F & $<0.056$ & $<0.014$ & $<0.005$ & $<0.21$ & $<0.006$ & $<0.007$ & $<0.03$ & $<0.63$ & $<0.07$ & $<0.14$ & $<0.001$ & $<0.004$ \\
\hline A1-total & & $<0.014$ & $<0.005$ & 2.51 & $<0.006$ & $<0.007$ & $<0.03$ & 3.13 & $<0.07$ & $<0.14$ & $<0.001$ & 0.020 \\
\hline B1 & $>18$ & & & & & & & & & & & 34 \\
\hline B1-1 & 10 & $<0.015$ & & & $<0.007$ & & & & & & & \\
\hline B1-2 & 5.6 & $<0.015$ & $<0.005$ & 0.69 & $<0.007$ & $<0.007$ & $<0.04$ & $<0.67$ & $<0.07$ & $<0.15$ & $<0.001$ & 0.009 \\
\hline B1-3 & 3.2 & 0.016 & $<0.005$ & 0.75 & 0.007 & $<0.007$ & $<0.04$ & $<0.67$ & 0.10 & $<0.15$ & $<0.001$ & 0.006 \\
\hline B1-4 & 1.8 & $<0.015$ & $<0.005$ & $<0.22$ & $<0.007$ & $<0.007$ & $<0.04$ & $<0.67$ & $<0.07$ & $<0.15$ & $<0.001$ & $<0.004$ \\
\hline B1-5 & 1.0 & $<0.0$ & $<0.005$ & 0.25 & $<0.007$ & $<0.007$ & $<0.04$ & $<0.67$ & $<0.07$ & $<0.15$ & $<0.001$ & $<0.004$ \\
\hline B1-6 & 0.56 & $<0.015$ & $<0.005$ & $<0.22$ & $<0.007$ & $<0.007$ & $<0.04$ & $<0.67$ & $<0.07$ & $<0.15$ & $<0.001$ & $<0.004$ \\
\hline B1-7 & 0.32 & $<0.015$ & $<0.005$ & $<0.22$ & $<0.007$ & $<0.007$ & $<0.04$ & $<0.67$ & $<0.07$ & $<0.15$ & $<0.001$ & $<0.004$ \\
\hline B1-8 & 0.18 & $<0.015$ & $<0.005$ & $<0.22$ & $<0.007$ & $<0.007$ & $<0.04$ & $<0.67$ & $<0.07$ & $<0.15$ & $<0.001$ & $<0.004$ \\
\hline B1-9 & 0.1 & $<0.015$ & $<0.005$ & $<0.22$ & $<0.007$ & $<0.007$ & 1.19 & $<0.67$ & $<0.07$ & $<0.15$ & $<0.001$ & $<0.004$ \\
\hline B1-10 & 0.056 & $<0.015$ & $<0.005$ & $<0.22$ & $<0.007$ & $<0.007$ & 0.46 & $<0.67$ & $<0.07$ & $<0.15$ & $<0.001$ & $<0.004$ \\
\hline $\mathrm{B} 1-\mathrm{F}$ & $<0.056$ & $<0.015$ & $<0.005$ & $<0.22$ & $<0.007$ & $<0.007$ & $<0.04$ & $<0.67$ & $<0.07$ & $<0.15$ & $<0.001$ & $<0.004$ \\
\hline B1-total & & 0.016 & $<0.005$ & 2.62 & 0.007 & $<0.007$ & 1.66 & $<0.67$ & 0.10 & $<0.15$ & $<0.001$ & 0.015 \\
\hline
\end{tabular}




\begin{tabular}{|c|c|c|c|c|c|c|c|c|c|c|c|c|}
\hline \multicolumn{13}{|c|}{$\begin{array}{l}\text { FACILITY \#1 - Copper/Beryllium Foundry and Machine Shop } \\
\text { MOUDI Impactor Size-Selective Air Sample Results for Total Particulate and Thirty-one Elements }\end{array}$} \\
\hline $\begin{array}{l}\text { Sample } \\
\text { Number }\end{array}$ & $\begin{array}{l}\text { Particle } \\
\text { Size } \\
(\mu \mathrm{m})\end{array}$ & $\begin{array}{c}\mathrm{Pb} \\
\text { Conc. } \\
\left(\mu g / m^{3}\right)\end{array}$ & $\begin{array}{c}\text { Li } \\
\text { Conc. } \\
\left(\mu \mathrm{g} / \mathrm{m}^{3}\right)\end{array}$ & $\begin{array}{c}\mathrm{Mg} \\
\text { Conc. } \\
\left(\mu \mathrm{g} / \mathrm{m}^{3}\right)\end{array}$ & $\begin{array}{l}\text { Mn } \\
\text { Conc. } \\
\left(\mu g / m^{3}\right)\end{array}$ & $\begin{array}{c}\text { Mo } \\
\text { Conc. } \\
\left(\mu \mathrm{g} / \mathrm{m}^{3}\right)\end{array}$ & $\begin{array}{c}\mathrm{Ni} \\
\text { Conc. } \\
\left(\mu \mathrm{g} / \mathrm{m}^{3}\right)\end{array}$ & $\begin{array}{c}\text { P } \\
\text { Conc. } \\
\left(\mu \mathrm{g} / \mathrm{m}^{3}\right)\end{array}$ & $\begin{array}{c}\mathrm{K} \\
\text { Conc. } \\
\left(\mu \mathrm{g} / \mathrm{m}^{3}\right)\end{array}$ & $\begin{array}{c}\text { Se } \\
\text { Conc. } \\
\left(\mu \mathrm{g} / \mathrm{m}^{3}\right)\end{array}$ & $\begin{array}{c}\mathrm{Ag} \\
\text { Conc. } \\
\left(\mu \mathrm{g} / \mathrm{m}^{3}\right)\end{array}$ & $\begin{array}{c}\mathrm{Sr} \\
\text { Conc. } \\
\left(\mu \mathrm{g} / \mathrm{m}^{3}\right)\end{array}$ \\
\hline C1-0 & $>18$ & 0.016 & $<0.006$ & 0.61 & 0.031 & $<0.008$ & 0.10 & $<0.71$ & $<0.07$ & $<0.15$ & $<0.002$ & $<0.005$ \\
\hline C1-1 & 10 & $<0.016$ & $<0.006$ & 0.73 & 0.030 & $<0.008$ & 0.09 & $<0.71$ & 0.09 & $<0.15$ & $<0.002$ & $<0.005$ \\
\hline $\mathrm{C} 1-2$ & 5.6 & $<0.016$ & $<0.006$ & 0.61 & 0.018 & $<0.008$ & $<0.04$ & $<0.71$ & $<0.08$ & $<0.15$ & $<0.002$ & 0.005 \\
\hline C1-3 & 3.2 & $<0.016$ & $<0.006$ & 0.69 & 0.022 & $<0.008$ & $<0.04$ & $<0.71$ & 0.12 & $<0.15$ & $<0.002$ & 0.005 \\
\hline $\mathrm{C}_{1-4}$ & 1.8 & $<0.016$ & $<0.006$ & 0.31 & 0.009 & $<0.008$ & $<0.04$ & $<0.71$ & $<0.08$ & $<0.15$ & $<0.002$ & $<0.005$ \\
\hline C1-5 & 1.0 & $<0.016$ & $<0.006$ & 0.75 & $<0.007$ & $<0.008$ & $<0.04$ & 1.42 & $<0.08$ & $<0.15$ & & \\
\hline$C_{1-6}$ & 0.56 & $<0.016$ & $<0.006$ & $<0.24$ & $<0.007$ & $<0.008$ & $<0.04$ & $<0.71$ & $<0.08$ & $<0.15$ & $<0.002$ & $<0.005$ \\
\hline C1-7 & 0.32 & $<0.016$ & $<0.006$ & $<0.24$ & $<0.007$ & $<0.008$ & $<0.04$ & $<0.71$ & $<0.08$ & $<0.15$ & $<0.002$ & $<0.005$ \\
\hline$C 1-8$ & 0.18 & $<0.016$ & $<0.006$ & 0.24 & $<0.007$ & $<0.008$ & $<0.04$ & 0.72 & $<0.08$ & $<0.15$ & 0.009 & $<0.005$ \\
\hline C1-9 & 0.1 & $<0.016$ & $<0.006$ & $<0.24$ & $<0.007$ & $<0.008$ & $<0.04$ & $<0.71$ & $<0.08$ & $<0.15$ & $<0.002$ & $<0.005$ \\
\hline C1-10 & 0.056 & $<0.016$ & $<0.006$ & $<0.24$ & $<0.007$ & $<0.008$ & $<0.04$ & $<0.71$ & $<0.08$ & $<0.15$ & $<0.002$ & $<0.005$ \\
\hline $\mathrm{C} 1-\mathrm{F}$ & $<0.056$ & $<0.016$ & $<0.006$ & $<0.24$ & $<0.007$ & $<0.008$ & $<0.04$ & $<0.71$ & $<0.08$ & $<0.15$ & $<0.002$ & $<0.005$ \\
\hline C1-total & & 0.016 & $<0.006$ & 3.94 & 0.110 & $<0.008$ & 010 & 2.14 & 021 & $<0.15$ & 0.009 & 0.022 \\
\hline A2-0 & $>18$ & 7 & 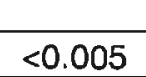 & 0 & $<0.006$ & & $<0.00$ & $<0.02$ & $<0.07$ & $<0.14$ & 0.001 & $<0.004$ \\
\hline A2-1 & 10 & $<0.014$ & $<0.005$ & 0.30 & $<0.006$ & $<0.007$ & $<0.03$ & $<0.62$ & $<0.07$ & $<0.14$ & $<0.001$ & $<0.004$ \\
\hline A2-2 & 5.6 & $<0.014$ & $<0.005$ & 0.66 & $<0.006$ & $<0.007$ & $<0.03$ & 1.17 & $<0.07$ & $<0.14$ & $<0.001$ & 0.008 \\
\hline A2-3 & 3.2 & $<0.014$ & $<0.005$ & 0.33 & $<0.006$ & $<0.007$ & $<0.03$ & $<0.62$ & $<0.07$ & $<0.14$ & $<0.001$ & $<0.004$ \\
\hline A2-4 & 1.8 & $<0.014$ & $<0.005$ & $<0.21$ & $<0.006$ & $<0.007$ & $<0.03$ & $<0.62$ & $<0.07$ & $<0.14$ & 0.002 & $<0.004$ \\
\hline A2-5 & 1.0 & $<0.014$ & $<0.005$ & $<0.21$ & $<0.006$ & $<0.007$ & $<0.03$ & $<0.62$ & $<0.07$ & $<0.14$ & $<0.001$ & $<0.004$ \\
\hline A2-6 & 0.56 & $<0.014$ & $<0.005$ & 0.23 & $<0.006$ & $<0.007$ & $<0.03$ & 0.76 & $<0.07$ & $<0.14$ & $<0.001$ & $<0.004$ \\
\hline A2-7 & 0.32 & $<0.014$ & $<0.005$ & $<0.21$ & $<0.006$ & $<0.007$ & $<0.03$ & $<0.62$ & $<0.07$ & $<0.14$ & $<0.001$ & $<0.004$ \\
\hline A2-8 & 0.18 & $<0.014$ & $<0.005$ & $<0.21$ & $<0.006$ & $<0.007$ & $<0.03$ & $<0.62$ & $<0.07$ & $<0.14$ & $<0.001$ & $<0.004$ \\
\hline A2-9 & 0.1 & $<0.014$ & $<0.005$ & 0.31 & $<0.006$ & $<0.007$ & $<0.03$ & 0.90 & $<0.07$ & $<0.14$ & $<0.001$ & 0.004 \\
\hline$A 2-10$ & 0.056 & $<0.014$ & $<0.005$ & $<0.21$ & $<0.006$ & $<0.007$ & $<0.03$ & $<0.62$ & $<0.07$ & $<0.14$ & $<0.001$ & $<0.004$ \\
\hline A2-F & $<0.056$ & $<0.014$ & $<0.005$ & $<0.21$ & $<0.006$ & $<0.007$ & $<0.03$ & $<0.62$ & $<0.07$ & $<0.14$ & $<0.001$ & $<0.004$ \\
\hline A2-total & & $<0.014$ & $<0.005$ & 2.31 & $<0.006$ & $<0.007$ & $<0.03$ & 2.83 & $<0.07$ & $<0.14$ & 0.002 & 0.012 \\
\hline
\end{tabular}




\begin{tabular}{|c|c|c|c|c|c|c|c|c|c|c|c|c|}
\hline \multicolumn{13}{|c|}{$\begin{array}{l}\text { FACILITY \#1 - Copper/Beryllium Foundry and Machine Shop } \\
\text { MOUDI Impactor Size-Selective Air Sample Results for Total Particulate and Thirty-one Elements }\end{array}$} \\
\hline $\begin{array}{l}\text { Sample } \\
\text { Number }\end{array}$ & $\begin{array}{l}\text { Particle } \\
\text { Size } \\
\text { (um) } \\
\end{array}$ & $\begin{array}{c}\mathrm{Pb} \\
\mathrm{Conc} \\
\left(\mu \mathrm{g} / \mathrm{m}^{3}\right)\end{array}$ & $\begin{array}{c}\text { Li } \\
\text { Conc. } \\
\left(\mu g / m^{3}\right)\end{array}$ & $\begin{array}{c}\mathrm{Mg} \\
\text { Conc. } \\
\left(\mu \mathrm{g} / \mathrm{m}^{3}\right)\end{array}$ & $\begin{array}{l}\text { Mn } \\
\text { Conc. } \\
\left(\mu g / m^{3}\right)\end{array}$ & $\begin{array}{c}\text { Mo } \\
\text { Conc. } \\
\left(\mu g / m^{3}\right)\end{array}$ & $\begin{array}{c}\mathrm{Ni} \\
\text { Conc. } \\
\left(\mu \mathrm{g} / \mathrm{m}^{3}\right)\end{array}$ & $\begin{array}{c}P \\
\text { Conc. } \\
\left(\mu g / m^{3}\right)\end{array}$ & $\begin{array}{c}K \\
\text { Conc. } \\
\left(\mu g / m^{3}\right)\end{array}$ & $\begin{array}{c}\text { Se } \\
\text { Conc. } \\
\left(\mu \mathrm{g} / \mathrm{m}^{3}\right)\end{array}$ & $\begin{array}{c}\mathrm{Ag} \\
\text { Conc. } \\
\left(\mu \mathrm{g} / \mathrm{m}^{3}\right)\end{array}$ & $\begin{array}{c}\mathrm{Sr} \\
\text { Conc. } \\
\left(\mu \mathrm{g} / \mathrm{m}^{3}\right)\end{array}$ \\
\hline $\mathrm{B} 2-0$ & $>18$ & $<0.014$ & $<0.005$ & 0.30 & $<0.006$ & $<0.007$ & $<0.04$ & $<0.63$ & $<0.07$ & $<0.14$ & $<0.001$ & $<0.004$ \\
\hline B2-1 & 10 & $<0.014$ & $<0.005$ & 0.32 & $<0.006$ & $<0.007$ & $<0.04$ & $<0.63$ & $<0.07$ & $<0.14$ & $<0.001$ & $<0.004$ \\
\hline B2-2 & 5.6 & $<0.014$ & $<0.005$ & 0.23 & $<0.006$ & $<0.007$ & $<0.04$ & $<0.63$ & $<0.07$ & $<0.14$ & $<0.001$ & $<0.004$ \\
\hline B2-3 & 3.2 & $<0.014$ & $<0.005$ & $<0.21$ & $<0.006$ & $<0.007$ & $<0.04$ & $<0.63$ & $<0.07$ & $<0.14$ & $<0.001$ & $<0.004$ \\
\hline B2-4 & 1.8 & $<0.014$ & $<0.005$ & 0.22 & $<0.006$ & $<0.007$ & $<0.04$ & $<0.63$ & $<0.07$ & $<0.14$ & $<0.001$ & $<0.004$ \\
\hline B2-5 & 1.0 & $<0.014$ & $<0.005$ & $<0.21$ & $<0.006$ & $<0.007$ & $<0.04$ & $<0.63$ & $<0.07$ & $<0.14$ & $<0.001$ & $<0.004$ \\
\hline B2-6 & 0.56 & $<0.014$ & $<0.005$ & 0.40 & $<0.006$ & $<0.007$ & $<0.04$ & 1.05 & $<0.07$ & $<0.14$ & $<0.001$ & 0.006 \\
\hline B2-7 & 0.32 & $<0.014$ & $<0.005$ & 0.23 & $<0.006$ & $<0.007$ & $<0.04$ & $<0.63$ & $<0.07$ & $<0.14$ & $<0.001$ & $<0.004$ \\
\hline B2-8 & $0 . \overline{18}$ & $<0.014$ & $<0.005$ & $<0.21$ & $<0.006$ & $<0.007$ & $<0.04$ & $<0.63$ & $<0.07$ & $<0.14$ & $<0.001$ & $<0.004$ \\
\hline B2-9 & 0.1 & $<0.014$ & $<0.005$ & $<0.21$ & $<0.006$ & $<0.007$ & $<0.04$ & $<0.63$ & $<0.07$ & $<0.14$ & $<0.001$ & $<0.004$ \\
\hline$B 2-10$ & 0.056 & $<0.014$ & $<0.005$ & $<0.21$ & $<0.006$ & $<0.007$ & 0.25 & $<0.63$ & $<0.07$ & $<0.14$ & $<0.001$ & $<0.004$ \\
\hline B2-F & $<0.056$ & $<0.014$ & $<0.005$ & $<0.21$ & $<0.006$ & $<0.007$ & $<0.04$ & $<0.63$ & $<0.07$ & $<0.14$ & $<0.001$ & $<0.004$ \\
\hline B2-total & & $<0.014$ & $<0.005$ & 1.69 & $<0.006$ & $<0.007$ & 0.25 & 1.05 & $<0.07$ & $<0.14$ & $<0.001$ & 0.006 \\
\hline & $>18$ & & & & & & & & & & & \\
\hline $\mathrm{C} 2-1$ & 10 & $<0.014$ & $<0.005$ & 0.23 & $<0.006$ & $<0$ & $<0.04$ & $<0.65$ & $<0.07$ & $<0.14$ & & $<0.004$ \\
\hline C2-2 & 5.6 & 0.015 & $<0.005$ & 0.46 & 0.013 & $<0.007$ & $<0.04$ & $<0.65$ & $<0.07$ & $<0.14$ & $<0.001$ & $<0.004$ \\
\hline $\mathrm{C} 2-3$ & 3.2 & $<0.014$ & $<0.005$ & 0.43 & 0.007 & $<0.007$ & $<0.04$ & $<0.65$ & $<0.07$ & $<0.14$ & $<0.001$ & $<0.004$ \\
\hline $\mathrm{C} 2-4$ & 1.8 & $<0.014$ & $<0.005$ & 0.27 & $<0.006$ & $<0.007$ & 0.10 & $<0.65$ & $<0.07$ & $<0.14$ & $<0.001$ & $<0.004$ \\
\hline C2-5 & 1.0 & $<0.014$ & $<0.005$ & 0.22 & 0.007 & $<0.007$ & $<0.04$ & $<0.65$ & $<0.07$ & $<0.14$ & $<0.001$ & $<0.004$ \\
\hline $\mathrm{C} 2-6$ & 0.56 & $<0.014$ & $<0.005$ & $<0.22$ & $<0.006$ & $<0.007$ & $<0.04$ & $<0.65$ & $<0.07$ & $<0.14$ & $<0.001$ & $<0.004$ \\
\hline $\mathrm{C} 2-7$ & 0.32 & $<0.014$ & $<0.005$ & $<0.22$ & $<0.006$ & $<0.007$ & $<0.04$ & $<0.65$ & $<0.07$ & $<0.14$ & $<0.001$ & $<0.004$ \\
\hline C2-8 & 0.18 & $<0.014$ & $<0.005$ & $<0.22$ & $<0.006$ & $<0.007$ & $<0.04$ & $<0.65$ & $<0.07$ & $<0.14$ & $<0.001$ & $<0.004$ \\
\hline C2-9 & $0 . \overline{1}$ & $<0.014$ & $<0.005$ & $<0.22$ & $<0.006$ & $<0.007$ & 0.05 & $<0.65$ & $<0.07$ & $<0.14$ & $<0.001$ & $<0.004$ \\
\hline $\mathrm{C} 2-10$ & $0.0 \overline{56}$ & $<0.014$ & $<0.005$ & $<0.22$ & $<0.006$ & $<0.007$ & $<0.04$ & $<0.65$ & $<0.07$ & $<0.14$ & $<0.001$ & $<0.004$ \\
\hline $\mathrm{C} 2-\mathrm{F}$ & $<0.056$ & $<0.014$ & $<0.005$ & $<0.22$ & $<0.006$ & $<0.007$ & $<0.04$ & $<0.65$ & $<0.07$ & $<0.14$ & $<0.001$ & $<0.004$ \\
\hline C2-total & & 0.015 & $<0.005$ & 2.06 & 0.041 & $<0.007$ & 0.20 & $<0.65$ & $<0.07$ & $<0.14$ & $<0.001$ & $<0.004$ \\
\hline
\end{tabular}




\section{Appendix D - continued}

FACILITY \#1 - Copper/Beryllium Foundry and Machine Shop MOUDI Impactor Size-Selective Air Sample Results for Total Particulate and Thirty-one Elements

\begin{tabular}{|c|c|c|c|c|c|c|c|c|c|}
\hline $\begin{array}{l}\text { Sample } \\
\text { Number }\end{array}$ & 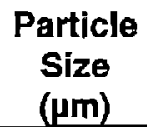 & $\begin{array}{c}\text { Te } \\
\text { Conc. } \\
\left(\mu \mathrm{g} / \mathrm{m}^{3}\right)\end{array}$ & $\begin{array}{c}\text { TI } \\
\text { Conc. } \\
\left(\mu g / \mathrm{m}^{3}\right)\end{array}$ & $\begin{array}{c}\text { Sn } \\
\text { Conc. } \\
\left(\mu g / m^{3}\right)\end{array}$ & $\begin{array}{c}\text { Ti } \\
\text { Conc. } \\
\left(\mu g / \mathrm{m}^{3}\right)\end{array}$ & $\begin{array}{c}V \\
\text { Conc. } \\
\left(\mathrm{\mu g} / \mathrm{m}^{3}\right)\end{array}$ & $\begin{array}{c}Y \\
\text { Conc. } \\
\left(\mu g / m^{3}\right)\end{array}$ & $\begin{array}{c}\mathrm{Zn} \\
\text { Conc. } \\
\left(\mu \mathrm{g} / \mathrm{m}^{3}\right)\end{array}$ & $\begin{array}{c}\mathrm{Zr} \text { Conc. } \\
\left(\mu \mathrm{g} / \mathrm{m}^{3}\right)\end{array}$ \\
\hline A1-0 & $>18$ & $<0.04$ & $<0.03$ & $<0.42$ & 0.004 & $<0.001$ & 0.0008 & $<0.07$ & $<0.04$ \\
\hline A1-1 & 10 & $<0.04$ & $<0.03$ & $<0.42$ & 0.003 & $<0.001$ & 0.0003 & $<0.07$ & $<0.04$ \\
\hline$A 1-2$ & 5.6 & $<0.04$ & $<0.03$ & $<0.42$ & 0.005 & $<0.001$ & $<0.0003$ & $<0.07$ & $<0.04$ \\
\hline A1-3 & 3.2 & $<0.04$ & $<0.03$ & 0.97 & 0.002 & $<0.001$ & $<0.0003$ & $<0.07$ & $<0.04$ \\
\hline A1-4 & 1.8 & $<0.04$ & $<0.03$ & 5.64 & 0.004 & $<0.001$ & $<0.0003$ & $<0.07$ & $<0.04$ \\
\hline A1-5 & 1.0 & $<0.04$ & $<0.03$ & 1.81 & 0.001 & $<0.001$ & $<0.0003$ & $<0.07$ & $<0.04$ \\
\hline A1-6 & 0.56 & $<0.04$ & $<0.03$ & 0.84 & $<0.001$ & $<0.001$ & $<0.0003$ & $<0.07$ & $<0.04$ \\
\hline A1-7 & 0.32 & $<0.04$ & $<0.03$ & 6.26 & 0.001 & $<0.001$ & $<0.0003$ & $<0.07$ & $<0.04$ \\
\hline A1-8 & 0.18 & $<0.04$ & $<0.03$ & 6.26 & 0.001 & $<0.001$ & $<0.0003$ & $<0.07$ & $<0.04$ \\
\hline A1-9 & 0.1 & $<0.04$ & $<0.03$ & 0.46 & $<0.001$ & $<0.001$ & $<0.0003$ & $<0.07$ & $<0.04$ \\
\hline$A 1-10$ & 0.056 & $<0.04$ & $<0.03$ & 0.58 & $<0.001$ & $<0.001$ & $<0.0003$ & $<0.07$ & $<0.04$ \\
\hline$A 1-F$ & $<0.056$ & $<0.04$ & $<0.03$ & $<0.42$ & $<0.001$ & $<0.001$ & $<0.0003$ & $<0.07$ & $<0.04$ \\
\hline A1-total & & $<0.04$ & $<0.03$ & 22.82 & 0.021 & $<0.001$ & 0.0011 & $<0.07$ & $<0.04$ \\
\hline B1-0 & $>18$ & $<0.04$ & $<0.04$ & 0.50 & 0.007 & $<0.001$ & $<0.0004$ & $<0.07$ & $<0.04$ \\
\hline $\mathrm{B} 1-1$ & 10 & $<0.04$ & $<0.04$ & 1.57 & 0.003 & $<0.001$ & $<0.0004$ & $<0.07$ & $<0.04$ \\
\hline B1-2 & 5.6 & $<0.04$ & $<0.04$ & 2.61 & 0.006 & $<0.001$ & $<0.0004$ & $<0.07$ & $<0.04$ \\
\hline B1-3 & 3.2 & $<0.04$ & $<0.04$ & 1.12 & 0.016 & $<0.001$ & $<0.0004$ & $<0.07$ & $<0.04$ \\
\hline B1-4 & 1.8 & $<0.04$ & $<0.04$ & $<0.45$ & 0.002 & $<0.001$ & $<0.0004$ & $<0.07$ & $<0.04$ \\
\hline B1-5 & 1.0 & $<0.04$ & $<0.04$ & $<0.45$ & 0.004 & $<0.001$ & $<0.0004$ & $<0.07$ & $<0.04$ \\
\hline $\mathrm{B} 1-6$ & 0.56 & $<0.04$ & $<0.04$ & $<0.45$ & 0.002 & $<0.001$ & $<0.0004$ & $<0.07$ & $<0.04$ \\
\hline $\mathrm{B} 1-7$ & 0.32 & $<0.04$ & $<0.04$ & 1.42 & 0.001 & $<0.001$ & $<0.0004$ & $<0.07$ & $<0.04$ \\
\hline B1-8 & 0.18 & $<0.04$ & $<0.04$ & 1.64 & 0.001 & $<0.001$ & $<0.0004$ & $<0.07$ & $<0.04$ \\
\hline B1-9 & 0.1 & $<0.04$ & $<0.04$ & $<0.45$ & 0.001 & $<0.001$ & $<0.0004$ & $<0.07$ & $<0.04$ \\
\hline $\mathrm{B} 1-10$ & 0.056 & $<0.04$ & $<0.04$ & 0.73 & $<0.001$ & $<0.001$ & $<0.0004$ & $<0.07$ & $<0.04$ \\
\hline $\mathrm{B} 1-\mathrm{F}$ & $<0.056$ & $<0.04$ & $<0.04$ & $<0.45$ & $<0.001$ & $<0.001$ & $<0.0004$ & $<0.07$ & $<0.04$ \\
\hline B1-total & & $<0.04$ & $<0.04$ & 9.58 & 0.042 & $<0.001$ & $<0.0004$ & $<0.07$ & $<0.04$ \\
\hline
\end{tabular}




\begin{tabular}{|c|c|c|c|c|c|c|c|c|c|}
\hline \multicolumn{10}{|c|}{$\begin{array}{l}\text { Appendix D - continued } \\
\text { FACILITY \#1 - Copper/Beryllium Foundry and Machine Shop } \\
\text { MOUDI Impactor Size-Selective Air Sample Results for Total Particulate and Thirty-one Elements }\end{array}$} \\
\hline $\begin{array}{l}\text { Sample } \\
\text { Number }\end{array}$ & $\begin{array}{l}\text { Particle } \\
\text { Size } \\
(\mu \mathrm{m})\end{array}$ & $\begin{array}{l}\text { Te } \\
\text { Conc. } \\
\left(\mu g / m^{3}\right)\end{array}$ & $\begin{array}{c}\text { TI } \\
\text { Conc. } \\
\left(\mu g / m^{3}\right)\end{array}$ & $\begin{array}{c}\text { Sn } \\
\text { Conc. } \\
\left(\mu g / \mathrm{m}^{3}\right)\end{array}$ & $\begin{array}{c}\text { Ti } \\
\text { Conc. } \\
\left(\mu g / m^{3}\right)\end{array}$ & $\begin{array}{c}V \\
\text { Conc. } \\
\left(\mu \mathrm{g} / \mathrm{m}^{3}\right)\end{array}$ & $\begin{array}{c}Y \\
\text { Conc } \\
\left(\mu g / \mathrm{m}^{3}\right)\end{array}$ & $\begin{array}{c}\text { Zn } \\
\text { Conc. } \\
\left(\mu g / \mathrm{m}^{3}\right)\end{array}$ & $\begin{array}{c}\mathrm{Zr} \text { Conc. } \\
\left(\mu \mathrm{g} / \mathrm{m}^{3}\right)\end{array}$ \\
\hline $\mathrm{C} 1-0$ & $>18$ & $<0.05$ & $<0.04$ & $<0.47$ & 0.013 & $<0.002$ & $<0.0004$ & $<0.08$ & $<0.05$ \\
\hline C1-1 & 10 & $<0.05$ & $<0.04$ & $<0.47$ & 0.016 & $<0.002$ & $<0.0004$ & $<0.08$ & $<0.05$ \\
\hline C1-2 & 5.6 & $<0.05$ & $<0.04$ & 0.94 & 0.009 & $<0.002$ & $<0.0004$ & $<0.08$ & $<0.05$ \\
\hline C1-3 & 3.2 & $<0.05$ & $<0.04$ & $<0.47$ & 0.012 & $<0.002$ & $<0.0004$ & $<0.08$ & $<0.05$ \\
\hline C1-4 & 1.8 & $<0.05$ & $<0.04$ & $<0.47$ & 0.006 & $<0.002$ & $<0.0004$ & $<0.08$ & $<0.05$ \\
\hline C1-5 & 1.0 & $<0.05$ & $<0.04$ & $<0.47$ & 0.003 & $<0.002$ & $<0.0004$ & $<0.08$ & $<0.05$ \\
\hline C1-6 & 0.56 & $<0.05$ & $<0.04$ & 1.81 & 0.001 & $<0.002$ & $<0.0004$ & $<0.08$ & $<0.05$ \\
\hline C1-7 & 0.32 & $<0.05$ & $<0.04$ & $<0.47$ & $<0.001$ & $<0.002$ & $<0.0004$ & $<0.08$ & $<0.05$ \\
\hline C1-8 & 0.18 & $<0.05$ & $<0.04$ & 3.46 & 0.001 & $<0.002$ & $<0.0004$ & $<0.08$ & $<0.05$ \\
\hline C1-9 & 0.1 & $<0.05$ & $<0.04$ & $<0.47$ & $<0.001$ & $<0.002$ & $<0.0004$ & $<0.08$ & $<0.05$ \\
\hline C1-10 & 0.056 & $<0.05$ & $<0.04$ & $<0.47$ & $<0.001$ & $<0.002$ & $<0.0004$ & $<0.08$ & $<0.05$ \\
\hline G1-F & $<0.056$ & $<0.05$ & $<0.04$ & $<0.47$ & $<0.001$ & $<0.002$ & $<0.0004$ & $<0.08$ & $<0.05$ \\
\hline C1-total & & $<0.05$ & $<0.04$ & 6.21 & 0.061 & $<0.002$ & $<0.0004$ & $<0.08$ & $<0.05$ \\
\hline A2-0 & $>18$ & $<0.04$ & $<0.03$ & 2.14 & 0.003 & $<0.001$ & $<0.0003$ & $<0.07$ & $<0.04$ \\
\hline A2-1 & 10 & $<0.04$ & $<0.03$ & $<0.41$ & 0.003 & $<0.001$ & $<0.0003$ & $<0.07$ & $<0.04$ \\
\hline A2-2 & 5.6 & $<0.04$ & $<0.03$ & 5.38 & 0.004 & $<0.001$ & $<0.0003$ & $<0.07$ & $<0.04$ \\
\hline A2-3 & 3.2 & $<0.04$ & $<0.03$ & 1.59 & 0.003 & $<0.001$ & $<0.0003$ & $<0.07$ & $<0.04$ \\
\hline A2-4 & 1.8 & $<0.04$ & $<0.03$ & 0.41 & 0.002 & $<0.001$ & $<0.0003$ & $<0.07$ & $<0.04$ \\
\hline A2-5 & 1.0 & $<0.04$ & $<0.03$ & $<0,41$ & $<0.001$ & $<0.001$ & $<0.0003$ & $<0.07$ & $<0.04$ \\
\hline A2 -6 & 0.56 & $<0.04$ & $<0.03$ & 2.69 & 0.001 & $<0.001$ & $<0.0003$ & $<0.07$ & $<0.04$ \\
\hline A2-7 & 0.32 & $<0.04$ & $<0.03$ & $<0.41$ & $<0.001$ & $<0.001$ & $<0.0003$ & $<0.07$ & $<0.04$ \\
\hline A2-8 & 0.18 & $<0.04$ & $<0.03$ & 2.62 & $<0.001$ & $<0.001$ & $<0.0003$ & $<0.07$ & $<0.04$ \\
\hline A2-9 & 0.1 & $<0.04$ & $<0.03$ & 4.42 & 0.001 & $<0.001$ & $<0.0003$ & $<0.07$ & $<0.04$ \\
\hline A2-10 & 0.056 & $<0.04$ & $<0.03$ & $<0.41$ & $<0.001$ & $<0.001$ & $<0.0003$ & $<0.07$ & $<0.04$ \\
\hline A2-F & $<0.056$ & $<0.04$ & $<0.03$ & 0.57 & $<0.001$ & $<0.001$ & $<0.0003$ & $<0.07$ & $<0.04$ \\
\hline A2-total & & $<0.04$ & $<0.03$ & 19.82 & 0.017 & $<0.001$ & $<0.0003$ & $<0.07$ & $<0.04$ \\
\hline
\end{tabular}




\begin{tabular}{|c|c|c|c|c|c|c|c|c|c|}
\hline \multicolumn{10}{|c|}{$\begin{array}{l}\text { FACILITY \#1 - Copper/Beryllium Foundry and Machine Shop } \\
\text { MOUDI Impactor Size-Selective Air Sample Results for Total Particulate and Thirty-one Element }\end{array}$} \\
\hline $\begin{array}{l}\text { Sample } \\
\text { Number }\end{array}$ & $\begin{array}{l}\text { Particle } \\
\text { Size } \\
(\mu \mathrm{m})\end{array}$ & $\begin{array}{c}\text { Te } \\
\text { Conc. } \\
\left(\mu \mathrm{g} / \mathrm{m}^{3}\right)\end{array}$ & $\begin{array}{c}\text { TI } \\
\text { Conc. } \\
\left(\mu \mathrm{g} / \mathrm{m}^{3}\right)\end{array}$ & $\begin{array}{c}\text { Sn } \\
\text { Conc. } \\
\left(\mu g / m^{3}\right)\end{array}$ & $\begin{array}{c}\mathrm{Ti} \\
\text { Conc. } \\
\left(\mu g / \mathrm{m}^{3}\right)\end{array}$ & $\begin{array}{l}v \\
\text { Conc. } \\
\left(\mu g / m^{3}\right)\end{array}$ & $\begin{array}{c}Y \\
\text { Conc. } \\
\left(\mu \mathrm{g} / \mathrm{m}^{3}\right)\end{array}$ & $\begin{array}{c}\mathrm{Zn} \\
\mathrm{Conc} \\
\left(\mu \mathrm{g} / \mathrm{m}^{3}\right)\end{array}$ & $\begin{array}{c}\mathrm{Zr} \text { Conc. } \\
\left(\mu \mathrm{g} / \mathrm{m}^{3}\right)\end{array}$ \\
\hline B2-0 & $>18$ & $<0.04$ & $<0.04$ & 2.53 & 0.003 & $<0.001$ & $<0.0004$ & $<0.07$ & $<0.04$ \\
\hline B2-1 & 10 & $<0.04$ & $<0.04$ & 1.20 & 0.005 & $<0.001$ & $<0.0004$ & $<0.07$ & $<0.04$ \\
\hline B2-2 & 5.6 & $<0.04$ & $<0.04$ & 0.43 & 0.003 & $<0.001$ & $<0.0004$ & $<0.07$ & $<0.04$ \\
\hline B2-3 & 3.2 & $<0.04$ & $<0.04$ & $<0.42$ & 0.002 & $<0.001$ & $<0.0004$ & $<0.07$ & $<0.04$ \\
\hline B2-4 & 1.8 & $<0.04$ & $<0.04$ & 2.32 & 0.001 & $<0.001$ & $<0.0004$ & $<0.07$ & $<0.04$ \\
\hline B2-5 & 1.0 & $<0.04$ & $<0.04$ & $<0.42$ & 0.001 & $<0.001$ & $<0.0004$ & $<0.07$ & $<0.04$ \\
\hline B2-6 & 0.56 & $<0.04$ & $<0.04$ & 5.20 & 0.001 & $<0.001$ & $<0.0004$ & $<0.07$ & $<0.04$ \\
\hline B2-7 & 0.32 & $<0.04$ & $<0.04$ & 2.32 & 0.001 & $<0.001$ & $<0.0004$ & $<0.07$ & $<0.04$ \\
\hline B2-8 & 0.18 & $<0.04$ & $<0.04$ & $<0.42$ & $<0.001$ & $<0.001$ & $<0.0004$ & $<0.07$ & $<0.04$ \\
\hline B2-9 & 0.1 & $<0.04$ & $<0.04$ & 2.25 & $<0.001$ & $<0.001$ & $<0.0004$ & $<0.07$ & $<0.04$ \\
\hline $\mathrm{B} 2-10$ & 0.056 & $<0.04$ & $<0.04$ & 0.60 & $<0.001$ & $<0.001$ & $<0.0004$ & $<0.07$ & $<0.04$ \\
\hline B2-F & $<0.056$ & $<0.04$ & $<0.04$ & $<0.42$ & $<0.001$ & $<0.001$ & $<0.0004$ & $<0.07$ & $<0.04$ \\
\hline B2-total & & $<0.04$ & $<0.04$ & 16.86 & $<0.001$ & $<0.001$ & $<0.0004$ & $<0.07$ & $<0.04$ \\
\hline $\mathrm{C} 2-0$ & $>18$ & $<0.04$ & & $<0$. & & & & & \\
\hline $\mathrm{C} 2-1$ & 10 & $<0.04$ & $<0.04$ & $<0.43$ & 0.006 & $<0.001$ & $<0.0004$ & $<0.07$ & $<0.04$ \\
\hline $\mathrm{C} 2-2$ & 5.6 & $<0.04$ & $<0.04$ & $<0.43$ & 0.012 & $<0.001$ & $<0.0004$ & $<0.07$ & $<0.04$ \\
\hline $\mathrm{C} 2-3$ & 3.2 & $<0.04$ & $<0.04$ & 0.79 & 0.011 & $<0.001$ & $<0.0004$ & $<0.07$ & $<0.04$ \\
\hline $\mathrm{C} 2-4$ & 1.8 & $<0.04$ & $<0.04$ & 1.94 & 0.005 & $<0.001$ & $<0.0004$ & $<0.07$ & $<0.04$ \\
\hline $\mathrm{C} 2-5$ & 1.0 & $<0.04$ & $<0.04$ & $<0.43$ & 0.004 & $<0.001$ & $<0.0004$ & $<0.07$ & $<0.04$ \\
\hline C2-6 & 0.56 & $<0.04$ & $<0.04$ & $<0.43$ & 0.001 & $<0.001$ & $<0.0004$ & $<0.07$ & $<0.04$ \\
\hline C2-7 & 0.32 & $<0.04$ & $<0.04$ & $<0.43$ & 0.001 & $<0.001$ & $<0.0004$ & $<0.07$ & $<0.04$ \\
\hline C2-8 & 0.18 & $<0.04$ & $<0.04$ & $<0.43$ & $<0.001$ & $<0.001$ & $<0.0004$ & $<0.07$ & $<0.04$ \\
\hline C2-9 & 0.1 & $<0.04$ & $<0.04$ & $<0.43$ & $<0.001$ & $<0.001$ & $<0.0004$ & $<0.07$ & $<0.04$ \\
\hline$C_{2}-10$ & 0.056 & $<0.04$ & $<0.04$ & $<0.43$ & $<0.001$ & $<0.001$ & $<0.0004$ & $<0.07$ & $<0.04$ \\
\hline $\mathrm{C} 2-\mathrm{F}$ & $<0.056$ & $<0.04$ & $<0.04$ & $<0.43$ & $<0.001$ & $<0.001$ & $<0.0004$ & $<0.07$ & $<0.04$ \\
\hline C2-total & & $<<0.04$ & $<<0.04$ & 2.74 & 0.052 & $<0.001$ & $<0.0004$ & $<<0.07$ & $<<0.04$ \\
\hline
\end{tabular}




\begin{tabular}{|c|c|c|c|c|c|c|c|c|c|c|c|c|c|c|c|c|}
\hline \multicolumn{17}{|c|}{ Appendix E } \\
\hline \multicolumn{17}{|c|}{$\begin{array}{l}\text { FACILITY \#1 - Copper/Beryllium Foundry and Machine Shop } \\
\text { Bulk Dust Sample Results for Thirty-one Elements }\end{array}$} \\
\hline $\begin{array}{l}\text { Sample } \\
\text { Number }\end{array}$ & $\begin{array}{c}\text { A } \\
\text { Conc. } \\
(\mathbf{m g} / \mathrm{Kg})\end{array}$ & $\begin{array}{c}\mathrm{Sb} \\
\text { Conc. } \\
(\mathrm{mg} / \mathrm{Kg})\end{array}$ & $\begin{array}{c}\text { As } \\
\text { Conc. } \\
(\mathrm{mg} / \mathrm{Kg})\end{array}$ & $\begin{array}{c}\mathrm{Ba} \\
\text { Conc. } \\
\text { (mg/Kg) }\end{array}$ & $\begin{array}{c}\text { Be } \\
\text { Conc. } \\
\text { (mg/Kg) }\end{array}$ & $\begin{array}{c}\text { Cd } \\
\text { Conc. } \\
\text { (mg/Kg) }\end{array}$ & $\begin{array}{l}\mathrm{Ca} \\
\text { Conc. } \\
(\mathrm{mg} / \mathrm{Kg})\end{array}$ & $\begin{array}{c}\mathrm{Cr} \\
\text { Conc. } \\
\text { (mg/Kg) }\end{array}$ & $\begin{array}{c}\text { Co } \\
\text { Conc. } \\
(\mathrm{mg} / \mathrm{Kg})\end{array}$ & $\begin{array}{c}\mathrm{Cu} \\
\text { Conc. } \\
(\mathrm{mg} / \mathrm{Kg})\end{array}$ & $\begin{array}{c}\text { Fe } \\
\text { Conc. } \\
\text { (mg/Kg) }\end{array}$ & $\begin{array}{c}\text { La } \\
\text { Conc. } \\
\text { (mg/Kg) }\end{array}$ & $\begin{array}{c}\mathrm{Pb} \\
\text { Conc. } \\
\text { (mg/Kg) }\end{array}$ & $\begin{array}{c}\mathrm{Ll} \\
\text { Conc. } \\
\text { (mg/Kg) }\end{array}$ & $\begin{array}{c}\mathrm{Mg} \\
\text { Conc. } \\
(\mathrm{mg} / \mathrm{Kg})\end{array}$ & $\begin{array}{c}\text { Mn } \\
\text { Conc. } \\
\text { (mg/Kg) }\end{array}$ \\
\hline SMWB-1 & 2000 & 3.9 & $<6$ & 79 & 940 & 12 & 5200 & 1700 & 480 & 54000 & 120000 & 31 & 110 & 30 & 1800 & 1200 \\
\hline SMWB-2 & 3600 & $<3$ & $<6$ & 5.7 & 2000 & $<0.9$ & 1300 & 23 & 3200 & 240000 & 1300 & 0.74 & 390 & 2.7 & 570 & 37 \\
\hline SMWB-3 & 12000 & 8.9 & $<6$ & 20 & 2500 & $<0.9$ & 12000 & 890 & 2400 & 220000 & 16000 & 4.9 & 430 & 11 & 7200 & 230 \\
\hline SMWB-4 & 19000 & 6.1 & 6.6 & 53 & 13000 & $<0.9$ & 18000 & 160 & 480 & 350000 & 12000 & 3.7 & 580 & 14 & 5800 & 200 \\
\hline \multirow[t]{2}{*}{ SMWB-5 } & 6900 & $<3$ & $<6$ & 7.3 & 100 & $<0.9$ & 940 & 9.5 & 97 & 11000 & 4500 & 3.6 & 26 & $<2$ & 460 & 48 \\
\hline & $\begin{array}{c}\text { Mo } \\
\text { Conc. } \\
\text { (mg/Kg) }\end{array}$ & $\begin{array}{c}\mathrm{NI} \\
\text { Conc. } \\
\text { (mg/Kg) }\end{array}$ & $\begin{array}{c}P \\
\text { Conc. } \\
\text { (mg/Kg) }\end{array}$ & $\begin{array}{c}\mathrm{K} \\
\text { Conc. } \\
\text { (mg/Kg) }\end{array}$ & $\begin{array}{c}\text { Se } \\
\text { Conc. } \\
\text { (mg/Kg) }\end{array}$ & $\begin{array}{c}\mathrm{Ag} \\
\text { Conc. } \\
\text { (mg/Kg) }\end{array}$ & $\begin{array}{c}\mathrm{Sr} \\
\text { Conc. } \\
\text { (mg/Kg) }\end{array}$ & $\begin{array}{c}\mathrm{Te} \\
\text { Conc. } \\
\text { (mg/Kg) }\end{array}$ & $\begin{array}{c}\mathrm{Tl} \\
\text { Conc. } \\
\text { (mg/Kg) }\end{array}$ & $\begin{array}{c}\text { Sn } \\
\text { Conc. } \\
\text { (mg/Kg) }\end{array}$ & $\begin{array}{c}\mathrm{Ti} \\
\text { Conc. } \\
\text { (mg/Kg) }\end{array}$ & $\begin{array}{c}\mathrm{V} \\
\text { Conc. } \\
\text { (mg/Kg) }\end{array}$ & $\begin{array}{c}\mathrm{Y} \\
\text { Conc. } \\
\text { (mg/Kg) }\end{array}$ & $\begin{array}{c}\mathrm{Zn} \\
\text { Conc. } \\
\text { (mg/Kg) }\end{array}$ & $\begin{array}{c}\mathrm{Zr} \\
\text { Conc. } \\
\text { (mg/Kg) }\end{array}$ & \\
\hline SMWB-1 & 70 & 1800 & $<20$ & 550 & $<6$ & 9.0 & 18 & 8.4 & 5.4 & $<6$ & 54 & 1.6 & 0.98 & 660 & $<\theta$ & \\
\hline SMWB-2 & $<1$ & 2700 & $<20$ & 190 & $<6$ & 37 & 16 & 6.4 & 8.2 & 160 & 30 & $<0.3$ & 0.59 & 1300 & $<10$ & \\
\hline SMWB-3 & 22 & 2600 & $<20$ & 760 & $<6$ & 38 & 23 & 4.1 & 7.0 & 86 & 140 & 4.8 & 2.2 & 1700 & $<10$ & \\
\hline SMWB-4 & 25 & 3000 & $<20$ & 1600 & 11 & 45 & 38 & 7.2 & 9.6 & 74 & 300 & 6.1 & 1.7 & 2300 & 54 & \\
\hline SMWB-5 & 2.3 & 120 & $<20$ & 110 & 6.5 & 3 & 8.0 & $<2$ & $<4$ & $<7$ & 59 & 1.5 & 0.46 & 89 & $<10$ & \\
\hline
\end{tabular}




\section{REFERENCES}

${ }^{1} 42$ CFR 85a [2002]. Public Health Service, HHS: occupational safety and health investigations of places of employment.

${ }^{2}$ NIOSH [1994]. NIOSH Manual of Analytical Methods, Method 7300, 4th rev. ed., Eller PM, ed. Cincinnati, OH: National Institute for Occupational Safety and Health, DHHS (NIOSH) Publication No. 94-113.

${ }^{3} 10$ CFR 850 [2003]. Department of Energy: chronic beryllium disease prevention program.

${ }^{4}$ ATSDR [2002]. Toxicological profile for beryllium. Atlanta, GA: U.S. Department of Health and Human Services, Public Health Service, Agency for Toxic Substances and Disease Registry.

${ }^{5}$ OSHA [1999]. OSHA Hazard Information Bulletins: Preventing Adverse Health Effects from Exposure to Beryllium on the Job. Hazard Information Bulletin no. 19990902.

${ }^{6}$ ACGIH [2008]. 2008 TLVs@ and BEIs@: threshold limit values for chemical substances and physical agents and biological exposure indices. Cincinnati, $\mathrm{OH}$ : American Conference of Governmental Industrial Hygienists.

${ }^{7}$ Maynard, A.D. and Jensen, P.A. [2001]. Aerosol Measurement in the Workplace. Aerosol Measurement ed. by Paul A. Baron and Klaus Willeke. Ch. 25, p779-799.

${ }^{8}$ ASTM [2002]. Standard practice for collection of settled dust samples using wipe sampling methods for subsequent determination of metals. West Conshohocken, PA: American Society for Testing and Materials International, Designation D 6966-03.

${ }^{9}$ NIOSH [1994]. NIOSH Manual of Analytical Methods, Method 7303, 4th rev. ed., Eller PM, ed. Cincinnati, OH: National Institute for Occupational Safety and Health, DHHS (NIOSH) Publication No. 94-113.

10 NIOSH [1994]. NIOSH Manual of Analytical Methods, Method 9110, 4th rev. ed., Eller PM, ed. Cincinnati, OH: National Institute for Occupational Safety and Health, DHHS (NIOSH) Publication No. 94-113.

${ }^{11}$ CFR. Code of Federal Regulations. Washington, DC: U.S. Government Printing Office, Office of the Federal Register.

${ }^{12}$ NARA [2008]. Executive Order 12196--Occupational safety and health programs for Federal employees. College Park, MD:U.S. National Archives and Records Administration. Available on-line at:

http://www.archives.gov/federal-register/codification/executive-order/12196.html. Accessed June 6, 2008.

${ }^{13}$ NIOSH [1992]. Recommendations for occupational safety and health: compendium of policy documents and statements. Cincinnati, OH: U.S. Department of Health and Human Services, Public Health Service, Centers for Disease Control and Prevention, National Institute for Occupational Safety and Health, DHHS (NIOSH) Publication No. 92-100.

${ }^{14}$ AlHA [2007]. 2007 Emergency Response Planning Guidelines (ERPG) \& Workplace Environmental Exposure Levels (WEEL) Handbook. Fairfax, VA: American Industrial Hygiene Association.

${ }^{15}$ NIOSH [2005]. NIOSH Pocket Guide to Chemical Hazards, Cincinnati, OH: U.S. Department of Health and Human Services, Public Health Service, Centers for Disease Control and Prevention, National Institute for Occupational Safety and Health, DHHS (NIOSH) Publication No. 2005-149. 
${ }^{16}$ Hathaway $\mathrm{G}$ et al, eds. [1991]. Proctor and Hughes' chemical hazards of the workplace, 3rd ed. New York, NY: Van Nostrand Reinhold.

${ }^{17}$ Caplan KJ [1993]. The significance of wipe samples. Am. Ind. Hyg. Assoc. J. 54:70-75.

${ }^{18}$ OSHA [2008] Surface Contamination Standards. Available on-line at http://www.osha.gov/SLTC/surfacecontamination/standards.html. Accessed May 12, 2008.

${ }^{19}$ NIOSH [2005]. NIOSH Respirator Selection Logic 2004, Cincinnati, OH: U.S. Department of Health and Human Services, Public Health Service, Centers for Disease Control and Prevention, National Institute for Occupational Safety and Health, DHHS (NIOSH) Publication No. 2005-100.

${ }^{20}$ ACGIH [2007]. INDUSTRIAL VENTILATION: A manual of Recommended Practice for Design, $26^{\text {th }}$ Edition. Cincinnati, OH: American Conference of Govemmental Industrial Hygienists, Committee on Industrial Ventilation. 